\title{
SINGULAR SUPPORT OF THE SCATTERING KERNEL FOR THE RAYLEIGH WAVE IN PERTURBED HALF-SPACES*
}

\author{
MISHIO KAWASHITA ${ }^{\dagger}$ AND HIDEO SOGA K $^{\ddagger}$
}

\begin{abstract}
This paper deals with the Rayleigh wave scattering on perturbed half-spaces in the framework of the Lax-Phillips type. Singular parts of the scattering kernel for this scattering are closely connected with singularities of the Rayleigh wave passing through the perturbation on the boundary. This can be described by estimating the singular support of the scattering kernel on the Rayleigh wave channel. The proof is based on a representation formula of the scattering kernel that was obtained in the previous work. However, the formula does not suit the situation of the Rayleigh wave, even though it is a natural extension of Majda's formula for the usual wave equation. Hence, the formula needs to be reformed, and the problem needs to be reduced to a pseudo-differential equation on the boundary governing the Rayleigh wave. Key methods for the reduced problem are construction of an approximate solution for the Rayleigh wave and analysis of an oscillatory integral distilled by using the solution. The phase function of the oscillatory integral is always degenerate along the characteristic curve of the Rayleigh wave. This degeneracy is handled by introducing a certain criterion for the regularity of the distribution defined by the oscillatory integral.
\end{abstract}

Key words. Scattering theory, distorted plane waves, scattering kernel, elastic wave equations, the Rayleigh wave.

AMS subject classifications. 35L20, 35P25, 74B05

1. Introduction. Let $\Omega$ be a perturbed space of the half-space $\mathbb{R}_{+}^{3}(=\{x=$ $\left.\left.\left(x^{\prime}, x_{3}\right)=\left(x_{1}, x_{2}, x_{3}\right) ; x_{3}>0\right\}\right)$ with a $C^{\infty}$ boundary $\Gamma$ (with bounded undulation), and consider the elastic wave equation

$$
\begin{cases}\left(\rho(x) \partial_{t}^{2}-\mathcal{A}\left(x, \partial_{x}\right)\right) u(t, x)=0 & \text { in } \mathbb{R} \times \Omega \\ \mathcal{N}\left(x, \partial_{x}\right) u(t, x)=0 & \text { on } \mathbb{R} \times \Gamma\end{cases}
$$

for the displacement vector $u(t, x)={ }^{t}\left(u_{1}(t, x), u_{2}(t, x), u_{3}(t, x)\right)$ of $x \in \Omega$ at time $t$. In (1.1), $\rho$ is the density of $\Omega, \mathcal{A} u=\sum_{i, j=1}^{3} \partial_{x_{i}}\left(a_{i j}(x) \partial_{x_{j}} u\right)$ is an operator with the coefficients $a_{i j}(x)$ of $3 \times 3$-matrix-valued functions, and $\mathcal{N}$ is the conormal derivative of $\mathcal{A}$ given by $\mathcal{N} u=\left.\sum_{i, j=1}^{3} \nu_{i}(x) a_{i j}(x) \partial_{x_{j}} u\right|_{\Gamma}$, where $\nu(x)={ }^{t}\left(\nu_{1}(x), \nu_{2}(x), \nu_{3}(x)\right)$ is the unit outer normal vector to $\Gamma$ at $x(\in \Gamma)$.

In this paper, the isotropic solids are considered; therefore, the $(p, q)$-components $a_{i p j q}(x)$ of $a_{i j}(x)$ are of the forms $a_{i p j q}(x)=\lambda(x) \delta_{i p} \delta_{j q}+\mu(x)\left(\delta_{i j} \delta_{p q}+\delta_{i q} \delta_{j p}\right)$, where $\delta_{i j}$ is Kronecker's delta, and $\lambda(x)$ and $\mu(x)$ are the Lamé parameters. We assume that the functions $\rho(x), \lambda(x)$, and $\mu(x)$ are $C^{\infty}$ in $\mathbb{R}^{3}$ and satisfy

$$
\inf _{x \in \bar{\Omega}} \rho(x)>0, \quad \inf _{x \in \bar{\Omega}}(\lambda(x)+2 \mu(x) / 3)>0, \quad \inf _{x \in \bar{\Omega}} \mu(x)>0 .
$$

Throughout this paper, we assume that there exists a constant $r_{0}>0$ such that the boundary $\Gamma$ consists of the boundary of the half-space outside the ball $B_{r_{0}}=$ $\left\{x \in \mathbb{R}^{3} ;|x|<r_{0}\right\}$ and that the functions $\rho(x), \lambda(x)$, and $\mu(x)$ are the constants $\rho_{0}$, $\lambda_{0}$, and $\mu_{0}$, respectively, outside $B_{r_{0}}$. Thus, outside $B_{r_{0}}, \mathcal{A}\left(x, \partial_{x}\right)$ is an operator with

\footnotetext{
${ }^{*}$ Received September 13, 2009; accepted for publication March 15, 2010.

$\dagger$ Department of Mathematics, Graduate School of Science, Hiroshima University, HigashiHiroshima, 739-8526 Japan (kawasita@math.sci.hiroshima-u.ac.jp). Partly supported by Grant-inAid for Science Research (C)(2) 19540183 from JSPS.

${ }^{\ddagger}$ Faculty of Education, Ibaraki University, Mito, Ibaraki, 310-8512, Japan (soga@mx.ibaraki. ac.jp). Partly supported by Grant-in-Aid for Science Research (C) 17540145 from JSPS.
} 
constant coefficients, and is denoted by $\mathcal{A}_{0}\left(\partial_{x}\right)$. Note that the system of the free space corresponding to (1.1) is given by replacing $\mathcal{A}$ with $\mathcal{A}_{0}$ and $\Omega$ with the half-space.

Two types of body waves exist for elastic waves: P-wave (the longitudinal wave) and $\mathrm{S}$-wave (the transversal wave). In the free system, the phase speeds of the $\mathrm{P}$ - and S-waves are given by $c_{P}=\sqrt{\left(\lambda_{0}+2 \mu_{0}\right) \rho_{0}^{-1}}$ and $c_{S}=\sqrt{\mu_{0} \rho_{0}^{-1}}$ respectively. There also exists a surface wave called the Rayleigh wave; this wave has the phase speed $c_{R}$ that is less than the speeds of the body waves, i.e. $0<c_{R}<c_{S}<c_{P}$. Note that the speed $c_{R}$ is determined by an algebraic equation (cf. (5.1) or [1]).

Since the Rayleigh wave is concentrated near the boundary, it has many interesting properties different from those of the body waves. In the case where the region $\Omega$ is an exterior domain with a compact boundary, the Rayleigh waves may cause strong trapping phenomena. For example, the local energy does not decay not quickly but rather slow (cf. [5] and [6]). Trapping phenomena often produce resonances. Stefanov and Vodev $[17,18]$ showed that the Rayleigh wave generates a sequence of resonances that approaches the real axis very quickly.

Since our interest is in scattering of the Rayleigh wave, it is natural to choose the half space $\mathbb{R}_{+}^{3}$ as the free space in scattering theory. In this case, the scattered Rayleigh wave may contain informations on the perturbation. We study how and what we can pick up from the scattering kernel associated with the Rayleigh wave.

According to this thought, M. Kawashita, W. Kawashita and Soga [7, 8] have formulated a scattering theory of the Lax-Phillips type for perturbed systems from the half space. In [7], the translation representations in the free space (the halfspace) are given. In [8], the theory for the perturbed space is developed, and the representation formula of the scattering kernel is obtained. Here, even the plane waves in the free space $\mathbb{R}_{+}^{3}$ contain the reflected waves consisting of different kinds of waves. Furthermore, the Rayleigh wave also exists. These are classified as follows:

(P) For the incident $\mathrm{P}$-wave, $\mathrm{P}$ - and $\mathrm{S}$-waves are reflected.

(SV) For the incident S-wave, P- and S-waves are reflected.

(SH) For the incident $\mathrm{S}$-wave, only the $\mathrm{S}$-wave is reflected.

(SVO) For the incident S-wave, the S-wave is totally reflected.

(R) The Rayleigh wave goes on the boundary.

Here, $\omega$ denotes the propagating direction of the reflected $\mathrm{P}$-waves in the case $(\mathrm{P})$, the reflected S-waves in the cases (SV), (SH), and (SVO), and the one of the Rayleigh wave in the case (R). Corresponding to these cases, the scattering kernel in our case is decomposed into the components $S_{\alpha \beta}(s, \theta, \omega)(\alpha, \beta=\mathrm{P}, \mathrm{SV}, \mathrm{SH}, \mathrm{SVO}, \mathrm{R})$. We call $S_{\alpha \beta}(s, \theta, \omega)$ the component for the channel of the incoming $\alpha$ and outgoing $\beta$.

The waves corresponding to the cases classified above are denoted by $w_{0}^{\alpha}(t, x ; \omega)$ $(\alpha=\mathrm{P}, \mathrm{SV}, \mathrm{SH}, \mathrm{SVO}, \mathrm{R})$. For each case the wave $w_{+, t o t}^{\alpha}(t, x ; \omega)$ exists in the perturbed space and is asymptotically equal to $w_{0}^{\alpha}(t, x ; \omega)$ as $t \rightarrow-\infty$. Note that these are the Fourier transforms of the generalized eigenfunctions in the free and perturbed spaces respectively (cf. [8]). Each $S_{\alpha \beta}(s, \theta, \omega)$ is represented by using $w_{0}^{\alpha}(t, x ; \theta)$ and $w_{+}^{\alpha}(t, x ; \omega)=w_{+, t o t}^{\alpha}(t, x ; \omega)-w_{0}^{\alpha}(t, x ; \omega)$. These representations correspond to Majda's formula [11] for scattering by obstacles in the scalar-valued wave equation. In the Majda case, the representation was expressed by using the plane waves of the form $\delta(t-x \cdot \omega)$ in the free space and the scattered waves for these waves. In our case, $w_{0}^{\alpha}(t, x ; \theta)$ correspond to the plane waves in the free space and $w_{+}^{\alpha}(t, x ; \omega)$ to the scattered waves. The represetation of $S_{\alpha \beta}(s, \theta, \omega)$ is stated in Theorem 2.2 (for the detail, see Theorem 6.1 of [8]). 
In this paper, we study singularities of the component for the channel of the Rayleigh wave (i.e. $S_{\mathrm{RR}}(s, \theta, \omega)$ ). Roughly speaking, this component has a meaning as follows: Let an incident (singular) Rayleigh wave come from the direction $\omega$ over a far distance and be scattered by the perturbation of the boundary; then, $S_{\mathrm{RR}}(s, \theta, \omega)$ means measurement of the scattered Rayleigh wave that goes eventually in the direction $\theta$. Our problem is to find where singularities of $S_{\mathrm{RR}}(s, \theta, \omega)$ appear and how they are connected with singularities of the Rayleigh wave.

This type of problem was studied by Majda [11] and others for the obstacle sattering of scalar-valued waves. Using his representation of the scattering kernel, Majda showed where the kernel was singular and proved that the convex hull of the obstacle was determined from these singularities. For scattering of elastic waves by compact obstacles, similar results were obtained by Soga [16] and others. These results are closely connected with the reflection phenomena of the plane waves by the obstacles, while the surface waves are excluded. Thus scattering of the Rayleigh wave itself has not yet been considered even though the Rayleigh wave may exist on the boundary.

According to Taylor [19] and others, singularities of the Rayleigh wave propagate only along the geodesics of the boundary $\Gamma$ with the phase speed $\tilde{c}_{R} \in C^{\infty}(\Gamma)$ (cf. section 5). In the microlocal sense, the original equation is reduced to a scalarvalued pseudo-differential equation of the real principal type on the boundary $\mathbb{R} \times \Gamma$ which governs the Rayleigh wave. This principal symbol is given by $\tilde{c}_{R}\left(x^{\prime}\right)\|\zeta\|_{\Gamma}-|\tau|$ $\left((t, \tau, \zeta) \in T^{*}(\mathbb{R}) \times T^{*}(\Gamma)\right)$, where $\|\zeta\|_{\Gamma}$ is the metric on $T^{*}(\Gamma)$ induced by the standard Riemann metric of $\Gamma$ (for details, see Proposition 5.1). Thus, we expect that the scattering phenomena of the Rayleigh wave are similar to those for the wave equation on the boundary $\Gamma$ if we only look at singularities and abandon other things than singularities.

Soga [14] investigated scattering for the scalar-valued wave equation with perturbation of the media in the Euclidian space. Based on his result and our expectation mentioned above, substituting the pseudo-differential equation governing the Rayleigh wave for the usual wave equation, we may guess the singularities of $S_{\mathrm{RR}}(s, \theta, \omega)$ appear similarly to those in [14]. As the final conclusion, this is correct, as is stated in Theorems 1 and 2. However, the proofs cannot be analogized with those of [14], which is because of the focus on the Rayleigh wave: The representation of $S_{\mathrm{RR}}(s, \theta, \omega)$ by M. Kawashita, W. Kawashita and Soga [8] is not of the integral form on the boundary where the Rayleigh wave is concentrated; this implies that the representation is not suitable only for analysis of the Rayleigh wave but also for explanation of the Rayleigh wave scattering, even though it is a natural extension of Majda's formula [11] to our case of elastic waves. Thus, using the differential equation in the unperturbed space for the Rayleigh wave and employing an approximate solution of the Rayleigh wave in the perturbed space, we reform the represetation to an appropriate form (cf. Theorem 3.1). This is the first step towards analyzing the scattering kernel for the surface waves like the Rayleigh wave, and is a crucial point. Note that this step is not needed when treating scattering of body waves (propagating inside the media).

The next step is to construct an approximate solution for the Rayleigh wave contained in the representation. Using this solution, we reduce the problem to the examination of an oscillatory integral. This seems to be the same approach as that by Majda [11], Soga [16], and others. However, in our case, even the construction of the approximate solution is not easy compared with the previous works of [11] and $[14,16]$. Furhtermore, the phase function of the oscillatory integral is always 
degenerate along the characteristic curve of the Rayleigh wave. This also makes a new difficulty. Thus, new techniques are needed to overcome these difficulties. Their development is another crucial point in this paper.

Let $\left(q\left(t, y^{\prime} ; \omega\right), p\left(t, y^{\prime} ; \omega\right)\right)$ be the phase flows in the cotangent bundle of $\Gamma$ for the Hamiltonian $\tilde{c}_{R}\left(x^{\prime}\right)\|\zeta\|_{\Gamma}$ with $\left(q\left(0, y^{\prime} ; \omega\right), p\left(0, y^{\prime} ; \omega\right)\right)=\left(y^{\prime}, c_{R}^{-1} \omega\right)$ for $\omega \in S^{1}$ and $y^{\prime}$ $\in \Gamma$ with $y^{\prime} \cdot \omega \leq-r_{0}$. We assume that these flows are non-trapping:

$$
\lim _{t \rightarrow \infty}\left|q\left(t, y^{\prime} ; \omega\right)\right|=\infty \quad \text { for any } \omega \in S^{1} \text { and } y^{\prime} \text { with } y^{\prime} \cdot \omega \leq-r_{0} .
$$

For $\omega$ and $\theta \in S^{1}$ we set

$$
\begin{aligned}
& M_{\omega}^{+}(\theta)=\left\{y^{\prime} \in \Gamma ; y^{\prime} \cdot \omega=-r_{0}, \lim _{t \rightarrow \infty} p\left(t+c_{R}^{-1} r_{0}, y^{\prime} ; \omega\right)=c_{R}^{-1} \theta\right\}, \\
& s_{\omega}^{+}(\theta)=\sup _{y^{\prime} \in M_{\omega}^{+}(\theta)} \lim _{t \rightarrow \infty}\left(c_{R}^{-1} q\left(t+c_{R}^{-1} r_{0}, y^{\prime} ; \omega\right) \cdot \theta-t\right),
\end{aligned}
$$

and denote the set $y^{\prime} \in M_{\omega}^{+}(\theta)$ attaining the supremum $s_{\omega}^{+}(\theta)$ by $M_{\omega}^{+, \max }(\theta)$, i.e., the maximal sojourn time. $M_{\omega}^{+}(\theta)$ and $s_{\omega}^{+}(\theta)$ are independent of the choice for $r_{0}$, and $M_{\omega}^{+, \max }(\theta) \neq \emptyset$ if $M_{\omega}^{+}(\theta) \neq \emptyset$. Note that these above limits exist since in our case the phase speed of the Rayleigh wave satisfies $\tilde{c}_{R}\left(x^{\prime}\right)=c_{R}$ outside of the ball $B_{r_{0}}$. The main results in this paper are the following theorems.

TheOrem 1.1. For any $\omega$ and $\theta \in S^{1}$, we have

(i) if $M_{\omega}^{+}(\theta)=\emptyset$, then $S_{\mathrm{RR}}(s, \theta, \omega)$ is $C^{\infty}$ on the whole of $\mathbb{R}_{s}$;

(ii) if $M_{\omega}^{+}(\theta) \neq \emptyset$, then sing $\operatorname{supp}\left[S_{\mathrm{RR}}(\cdot, \theta, \omega)\right] \subset\left(-\infty, s_{\omega}^{+}(\theta)\right]$.

For a tempered distribution $f \in \mathcal{S}^{\prime}(\mathbb{R})$ and $s_{0} \in \mathbb{R}$, we say that $f \in H^{l}$ at $s=s_{0}$, if there exists a cutoff function $\varphi \in C_{0}^{\infty}(\mathbb{R})$ such that $\varphi=1$ near $s_{0}$ and $\varphi f \in H^{l}(\mathbb{R})$.

THEOREM 1.2. If $M_{\omega}^{+, \max }(\theta)$ consists of only one point, $S_{\mathrm{RR}}(\cdot, \theta, \omega) \notin H^{-1}$ at $s=s_{\omega}^{+}(\theta)$.

Note that Theorem 1.2 implies the following corollary:

Corollary 1.3. If $M_{\omega}^{+, \max }(\theta)$ consists of only one point, $S_{\mathrm{RR}}(s, \theta, \omega)$ is singular at $s=s_{\omega}^{+}(\theta)$.

In [14], the results corresponding to Theorem 1.1 and Corollary 1.3 were obtained by assuming that bunches of paths of the geometrical optics near the paths attaining the maximal sojourn time did not make caustics. In the present paper, this assumption is removed. Instead, we assume that the paths attaining the maximum are unique. Assumptions of this type are needed to avoid some cancellation of the principal part (for detail see section 6). Even for the usual wave equation, we need these conditions to avoid cancellations since the principal part contains the terms from the Maslov index if caustics exist, which may cause the cancellations.

We now describe a rough sketch of the proofs. The first step is to reform the representation of the scattering kernel $S_{\mathrm{RR}}(s, \theta, \omega)$. As is explained earlier (also see Theorem 2.2 in section 2), the obtained representation of $S_{R R}(s, \theta, \omega)$ does not suit the scattering phenomena of the surface waves. Hence, we have to pick the main parts from the formula that contain essential information for the Rayleigh wave. For this purpose, we need the two steps divided into sections 2 and 3. Note that in usual cases, these steps are not needed.

We choose any point $s_{0} \in \mathbb{R}$ and localize the original formula of the scattering kernel stated in Theorem 2.2 at $s_{0}$. The scattering processes can be time-reversed if 
only singularities are focused on. In section 2 , using this fact, we reduce this localized formula near $s=s_{0}$ to a simple one (cf. Proposition 2.5). For this purpose, we introduce approximate solutions for the time reversed system described in (2.8) and (2.9). Note that even in the case of the usual wave equations this reduced formula seems to make the proofs simpler.

In section 3, further reduction suitable to the Rayleigh wave is given (cf. Theorem 3.1) by introducing another approximate solution with the Dirichlet boundary condition (cf. (3.1) and (3.2)). In this reduction, properties of the Rayleigh wave are used essentially. As a result, we have a reduced formula of $S_{R R}(s, \theta, \omega)$ localized at each point $s_{0}$, which consists of only one integral on the boundary $\mathbb{R} \times \Gamma$. This formula is reasonable and consistent with the scattering phenomena of the Rayleigh wave since it travels on the boundary. Moreover, this also has a technical advantage. Since the original formula consists of many integrals both in $\mathbb{R} \times \Omega$ and on $\mathbb{R} \times \partial\left(\Omega \cap \mathbb{R}_{+}^{3}\right)$, it is difficult to verify that these terms do not cancel each other out. To avoid this difficulty, we need these reductions.

The next step of the proof is to actually construct the approximate solutions introduced in sections 2 and 3. This step is divided into two parts. The data producing singularities of the scattered Rayleigh wave is contained in some part of the initial waves in the free space. First we have to pick up this part, which is done by introducing the approximate solutions of the equation with the zero Dirichlet boundary data and the non homogeneous data in $\Omega$. (cf. (4.1)). These approximate solutions are constructed in section 4 . In section 5 , the approximate solution containing scattered Rayleigh waves is constructed.

As shown in section 4, using the approximate solutions of (4.1) mentioned above, we can select the actual data as an inhomogeneous Neumann data for the equation of the scattered Rayleigh waves (cf. Proposition 4.5 and (4.2)). The approximate solution introduced in section 3 is also constructed by the same manner. Note that the arguments in section 4 do not contain construction of the scattered Rayleigh waves. Nevertheless, it is difficult to directly pick up the inhomogeneous boundary data mentioned above since the Rayleigh wave in the free space is very complex. Fortunately, the essential data are given by the commutator of $\rho_{0}^{-1} \mathcal{A}_{0}\left(\partial_{x}\right)$ and a cutoff function, and consequently, we can perform the whole calculation using a structure of the symbols for the elastic equations (cf. Lemma 4.4).

The approximate solution containing the scattered Rayleigh wave is governed by the equation with the inhomogeneous Neumann boundary data and the zero homogeneous data in $\Omega$ (cf. (4.2)). Construction of this solution is reduced to making asymptotic solutions of the Neumann operator that is restricted in the elliptic region of $\rho \partial_{t}^{2}-\mathcal{A}$ : this basically follows the approach due to Taylor [19]. In the last part of section 5 , we study the relations between the phase functions and sojourn times used in the following sections.

In section 6 , inserting the approximate solutions into the reduced representation of the kernel, we prove the main theorems in a more precise form (cf. Theorem 6.3). The proof is based on analysis of singularities for some distributions that are defined by oscillatory integrals with a parameter. In our case, the phase functions in these oscillatory integrals are always degenerate along the path of the maximal sojourn time. Hence, the stationary phase method does not work well. In [15], Soga gave sufficient conditions so that oscillatory integrals with degenerate phase functions did not rapidly decrease. In our case, however, these conditions are not satisfied. Thus, we need to find another criterion and improve Soga's method [15]. Our efforts are 
presented in section 7. As a result, we can measure the singularities of a class of distributions defined by oscillatory integrals in the sense of the usual Sobolev space (cf. Theorems 7.1 and 7.2).

In the last part of section 6 , we consider the case where the wave front of the Rayleigh wave going through the perturbation becomes strictly convex near whole paths corresponding to the maximal sojourn time. In this case, using the change in variables, we can reduce the oscillatory integrals to the forms to which the stationary phase method can be applied. Thus, the asymptotic form of $S_{R R}(s, \theta, \omega)$ is obtained (cf. Theorem 6.4). In this procedure, a kind of non-degenerate condition is introduced (cf. (6.17)). To the best of our knowledge, this non-degenerate condition has not previously been introduced, even for the usual wave equations. Noting Theorem 6.3 and Theorem 6.4, we can see that the present non-degenerate case is the weakest concerning singularities of the scattering kernels at the maximal sojourn time.

2. Reforming the kernel representation. In this section, we reform the representation of the scattering kernel obtained in [8]. Before doing so, we describe the notations and the basic results.

$H^{m}(M)$ denotes the Sobolev space of order $m$ on $M$. The mapping $U(t)$ : ${ }^{t}\left(u(0, \cdot), \partial_{t} u(0, \cdot)\right) \mapsto{ }^{t}\left(u(t, \cdot), \partial_{t} u(t, \cdot)\right)$ becomes a unitary operator on the Hilbert space $H=\dot{H}^{1}(\Omega) \times L^{2}(\Omega)$ with the inner product: $(f, g)_{H}=2^{-1}\left\{\sum_{i, j=1}^{3} \int_{\Omega} a_{i j} \partial_{x_{j}} f_{1}\right.$. $\left.\overline{\partial_{x_{i}} g_{1}} \rho d x+\int_{\Omega} f_{2} \cdot \overline{g_{2}} \rho d x\right\}\left(f={ }^{t}\left(f_{1}, f_{2}\right), g={ }^{t}\left(g_{1}, g_{2}\right)\right)$, where $\dot{H}^{m}(\Omega)=\{v(x) \in$ $H_{l o c}^{m}(\Omega): \partial_{x}^{\alpha} v \in L^{2}(\Omega)$ for $\left.1 \leq|\alpha| \leq m, \lim _{r \rightarrow \infty} r^{-2} \int_{r \leq|x| \leq 2 r}|v|^{2} d x=0\right\}$. The family $\{U(t)\}_{t \in \mathbb{R}}$ is a one parameter group of unitary operators with the generator $L$ of the form $L f={ }^{t}\left(f_{2}, \mathcal{A} f_{1}\right), f \in D(L)=\dot{H}_{\mathcal{N}}^{2}(\Omega) \times H^{1}(\Omega)$, where $\dot{H}_{\mathcal{N}}^{2}(\Omega)=\left\{v(x) \in \dot{H}^{2}(\Omega) ; \mathcal{N} v=0\right\}$ (cf. [8]).

M. Kawashita, W. Kawashita and Soga [8] have shown that we have the outgoing and incoming translation representations $T^{+}$and $T^{-}$in the Lax-Phillips sense (cf. [10]). These representations are unitary operators from $H$ to $L^{2}(\mathbb{R} ; N)$. Here, $N$ is of the form $N=\oplus_{\alpha \in \Lambda} L^{2}\left(S_{\alpha}\right)(\Lambda=\{\mathrm{P}, \mathrm{SV}, \mathrm{SH}, \mathrm{SVO}, \mathrm{R}\})$, and each $S_{\alpha}$ implies the set of all directions connected with the incident (or incoming) waves corresponding to the phenomena $\alpha$ described in the Introduction (for details, see [8]). In the Lax-Phillips theory, the scattering operator $S$ is defined by $S=T^{+}\left(T^{-}\right)^{-1} . S$ is a unitary operator on $L^{2}(\mathbb{R} ; N)$ and is expressed with a distribution kernel $S(s, \theta, \omega)$ : $(S k)(s, \theta)=k(s, \theta)+\int_{\mathbb{R}} S(s-\tilde{s}, \theta, \tilde{\omega}) k(\tilde{s}, \tilde{\omega}) d \tilde{s} d \tilde{\omega} \quad\left(k(\tilde{s}, \tilde{\omega}) \in L^{2}(\mathbb{R} ; N)\right) . S(s, \theta, \omega)$ is called the scattering kernel. The scattering kernel $S(s, \theta, \omega)$ consists of $5 \times 5$ matrices $\left(S_{\alpha \beta}(s, \theta, \omega)\right)_{\alpha, \beta \in \Lambda}$.

In [7], considering the reduced wave equation $\left(\sigma^{2}+\rho^{-1} \mathcal{A}\right) u=0$ with the constant coefficients, we have introduced the following plane incident waves $\phi_{0}^{\alpha, i}(\alpha=\mathrm{P}, \mathrm{SV}$, $\mathrm{SH}, \mathrm{SVO}$ ) and their reflected (or the totally reflected) waves $\phi_{0}^{\alpha, r}$ :

$$
\begin{aligned}
\phi_{0}^{P, i}(x ; \sigma, \omega) & =e^{i \sigma c_{P}^{-1} \check{\omega} \cdot x} a_{P}(\check{\omega}), & \phi_{0}^{S V O, i}(x ; \sigma, \omega) & =\gamma(\sigma, \omega) e^{i \sigma c_{S}^{-1} \breve{\omega} \cdot x} a_{S V}(\check{\omega}), \\
\phi_{0}^{S V, i}(x ; \sigma, \omega) & =e^{i \sigma c_{S}^{-1} \check{\omega} \cdot x} a_{S V}(\check{\omega}), & \phi_{0}^{S H, i}(x ; \sigma, \omega) & =e^{i \sigma c_{S}^{-1} \check{\omega} \cdot x} a_{S H}(\check{\omega}),
\end{aligned}
$$

where $\check{\omega}={ }^{t}\left(\omega^{\prime},-\omega_{3}\right), \omega^{\prime}={ }^{t}\left(\omega_{1}, \omega_{2}\right), a_{P}(\xi)=\xi={ }^{t}\left(\xi^{\prime}, \xi_{3}\right), a_{S V}(\xi)={ }^{t}\left(-\left(\xi_{3} /\left|\xi^{\prime}\right|\right) \xi^{\prime}\right.$, $\left.\left|\xi^{\prime}\right|\right), a_{S H}(\xi)=\left(1 /\left|\xi^{\prime}\right|\right)^{t}\left(-\xi_{2}, \xi_{1}, 0\right)$ and $\gamma(\sigma, \omega)$ is a certain function constant for $\sigma>0$ and for $\sigma<0$ (for details, see [7]). Here, we use $\omega$ in the notation $\phi_{0}^{\alpha, i}(x ; \sigma, \omega)$ to note the propagation direction by $\check{\omega}$, since this simplifies description of the calculations. We use the following generalized eigenfunctions:

$$
\phi_{0}^{\alpha}(x ; \sigma, \omega)=\phi_{0}^{\alpha, i}(x ; \sigma, \omega)+\phi_{0}^{\alpha, r}(x ; \sigma, \omega) \quad(\alpha \in \Lambda \backslash\{\mathrm{R}\}) .
$$


We also use the generalized eigenfunction corresponding to the Rayleigh wave:

$$
\phi_{0}^{R}(x ; \sigma, \omega)=\sqrt{2 \pi \rho_{0}} C_{0}^{R} e^{i \sigma c_{R}^{-1} \omega \cdot x^{\prime}} \sum_{j=1}^{2} C_{j}^{R} e^{-|\sigma| c_{R}^{-1} \xi_{R}^{(j)} x_{3}} a_{R}^{(j)}(\sigma, \omega),
$$

where $\omega \in S^{1}$ is the propagation direction and $\xi_{R}^{(1)}=\left\{1-\left(c_{R} / c_{P}\right)^{2}\right\}^{1 / 2}, \xi_{R}^{(2)}=$ $\left\{1-\left(c_{R} / c_{S}\right)^{2}\right\}^{1 / 2}, C_{1}^{R}=2-\left(c_{R} / c_{S}\right)^{2}, C_{2}^{R}=-2 \xi_{R}^{(1)}, a_{R}^{(1)}(\sigma, \omega)={ }^{t}\left(\omega,(i \sigma /|\sigma|) \xi_{R}^{(1)}\right)$, $a_{R}^{(2)}(\sigma, \omega)={ }^{t}\left(\xi_{R}^{(2)} \omega, i \sigma /|\sigma|\right)$, and the positive constant $C_{0}^{R}$ is taken so that

$$
|\sigma|\left(2 \pi \rho_{0} c_{R}\right)^{-1} \int_{0}^{\infty}\left|\phi_{0}^{R}(x ; \sigma, \omega)\right|^{2} d x_{3}=1 .
$$

We need this system of the generalized eigenfunction to represent the scattering kernel.

We set

$$
w_{0}^{\alpha}(t, x ; \omega)=(2 \pi)^{-1} \int_{\mathbb{R}} e^{i \sigma t} \phi_{0}^{\alpha}(x ;-\sigma, \omega) d \sigma \quad(\alpha \in \Lambda)
$$

Then, for $\alpha=\mathrm{P}, \mathrm{SV}, \mathrm{SH}, \mathrm{SVO}, w_{0}^{\alpha}(t, x ; \omega)$ is of the Dirac delta function type; it represents the reflection phenomena for the incident wave going in the direction $\check{\omega}$ in the half-space. $w_{0}^{R}(t, x ; \omega)$ is the Rayleigh wave of the same type on the boundary going in the direction $\omega$. Note that the form of $w_{0}^{R}(t, x ; \omega)$ given in section 1 is obtained by calculating the above Fourier integral.

We take a cutoff function $\psi \in C^{\infty}\left(\mathbb{R}^{3}\right)$ satisfying $0 \leq \psi \leq 1, \psi(x)=1$ for $|x|>r_{0}+5 / 3$ and $\psi(x)=0$ for $|x|<r_{0}+4 / 3$. For each $w_{0}^{\alpha}(t, x ; \omega)$, there exists a solution $w_{ \pm, t o t}^{\alpha}(t, x ; \omega) \in C^{\infty}\left(\bar{\Omega} ; \mathcal{S}^{\prime}\left(\mathbb{R}_{t}\right)\right)$ uniquely in the perturbed space (for the equation (1.1) $)$ such that $w_{ \pm, t o t}^{\alpha}(t, x ; \omega)-\psi(x) w_{0}^{\alpha}(t, x ; \omega)$ satisfies the following (+)condition (for details, see section 5 of [8]).

Definition 2.1. ( $( \pm)$-condition) We say that the solutions $v_{ \pm}(t, x)$ of the equation (1.1) satisfy the ( \pm )-condition ( respectively) if there exists a constant $t_{0}>0$ such that

$$
\begin{aligned}
& v_{ \pm} \in C^{\infty}\left(I_{t_{0}}^{\mp} ; \dot{H}^{\infty}(\Omega)\right) \text { with } \partial_{t} v_{ \pm} \in C^{\infty}\left(I_{t_{0}}^{\mp} ; H^{\infty}(\Omega)\right) \text { and } \\
& \lim _{t \rightarrow \mp \infty} \sum_{|\gamma|=1}\left\|\partial_{(t, x)}^{\gamma} v_{ \pm}(t, \cdot)\right\|_{L^{2}(\Omega)}=0,
\end{aligned}
$$

where $I_{t_{0}}^{-}=\left(-\infty,-t_{0}\right]$ and $I_{t_{0}}^{+}=\left[t_{0}, \infty\right)$.

Note that for $w_{ \pm, t o t}^{\alpha}(t, x ; \omega)-\psi(x) w_{0}^{\alpha}(t, x ; \omega)$, it suffices to choose $t_{0}=c_{R}^{-1}\left(r_{0}+3\right)$. The leading part (singular support) of $w_{0}^{\alpha}(t, x ; \omega)$ goes out of $B_{r_{0}+2}$ as $|t| \rightarrow \infty$, and therefore, roughly speaking, the wave

$$
w_{+}^{\alpha}(t, x ; \omega) \equiv w_{+, t o t}^{\alpha}(t, x ; \omega)-w_{0}^{\alpha}(t, x ; \omega)
$$

restricted in $\Omega \cap \mathbb{R}_{+}^{3}$ means the outgoing scattered wave for $w_{0}^{\alpha}(t, x ; \omega)$ in the free space.

The components $S_{\alpha \beta}(s, \theta, \omega)(\alpha, \beta \in \Lambda)$ of the scattering kernel are represented as follows using $w_{+}^{\alpha}(t, x ; \omega)$ and $w_{0}^{\alpha}(t, x ; \omega)$.

TheOREM 2.2. For $(\alpha, \beta \in \Lambda)$, we put $\tilde{S}_{\alpha \beta}(s, \theta, \omega)=$ $2(-2 \pi i)^{2}\left(c_{\alpha} c_{\beta}\right)^{3 / 2} \rho_{0} S_{\alpha \beta}(s, \theta, \omega)$, where $c_{S V}=c_{S H}=c_{S V O}=c_{S}$. Then we 
have

$$
\begin{array}{r}
\tilde{S}_{\alpha \beta}(s, \theta, \omega)=\int_{\Omega \cap \mathbb{R}_{+}^{3}} \int_{\mathbb{R}}\left(\partial_{s^{\prime}}^{2}-\rho_{0}^{-1} \mathcal{A}_{0}\left(\partial_{y}\right)\right) w_{+}^{\beta}\left(s^{\prime}-s, y ; \omega\right) \cdot \partial_{s^{\prime}} w_{0}^{\alpha}\left(s^{\prime}, y ; \theta\right) \rho_{0} d s^{\prime} d y \\
+\int_{\partial\left(\Omega \cap \mathbb{R}_{+}^{3}\right)}\left\{\int_{\mathbb{R}}\left(\tilde{\mathcal{N}}_{0}\left(\partial_{y}\right) w_{+}^{\beta}\right)\left(s^{\prime}-s, y ; \omega\right) \cdot \partial_{s^{\prime}} w_{0}^{\alpha}\left(s^{\prime}, y ; \theta\right) d s^{\prime}\right. \\
\left.\quad-\int_{\mathbb{R}} w_{+}^{\beta}\left(s^{\prime}-s, y ; \omega\right) \cdot \tilde{\mathcal{N}}_{0}\left(\partial_{y}\right) \partial_{s^{\prime}} w_{0}^{\alpha}\left(s^{\prime}, y ; \theta\right) d s^{\prime}\right\} d S_{y},
\end{array}
$$

where $\tilde{\mathcal{N}}_{0}\left(\partial_{y}\right) u=\mathcal{N}_{0}\left(\partial_{y}\right) u$ on $\partial \mathbb{R}_{+}^{3}$ and $\tilde{\mathcal{N}}_{0}\left(\partial_{y}\right) u=\sum_{i, j=1}^{3} \nu_{i}(y) a_{i j}^{0} \partial_{y_{j}} u$ on $\Gamma$.

Theorem 2.2 was proved by W. Kawashita, M. Kawashita and Soga [8] (cf. Theorem 6.1 of $[8])$.

We first rewrite the expression of $\tilde{S}_{\alpha \beta}(s, \theta, \omega)$ in Theorem 2.2 as follows:

Proposition 2.3. Let $\psi(x)$ be a $C^{\infty}$ cutoff function satisfying $0 \leq \psi \leq 1$, $\psi(x)=1$ for $|x|>r_{0}+5 / 3$ and $\psi(x)=0$ for $|x|<r_{0}+4 / 3$. Then for $\alpha, \beta \in \Lambda$ we have

$$
\begin{aligned}
& \tilde{S}_{\alpha \beta}(s, \theta, \omega)= \\
& \quad-\int_{\Omega} \int_{\mathbb{R}} w_{+, t o t}^{\beta}\left(s^{\prime}-s, y ; \omega\right) \cdot\left(\partial_{s^{\prime}}^{2}-\rho_{0}^{-1} \mathcal{A}_{0}\left(\partial_{y}\right)\right)\left(\psi \partial_{s^{\prime}} w_{0}^{\alpha}\right)\left(s^{\prime}, y ; \theta\right) d s^{\prime} \rho_{0} d y \\
& \quad-\int_{\Gamma} \int_{\mathbb{R}} w_{+, t o t}^{\beta}\left(s^{\prime}-s, y ; \omega\right) \cdot \mathcal{N}_{0}\left(\partial_{y}\right)\left(\psi \partial_{s^{\prime}} w_{0}^{\alpha}\right)\left(s^{\prime}, y ; \theta\right) d s^{\prime} d S_{y} .
\end{aligned}
$$

Proof. We decompose $\partial_{s^{\prime}} w_{0}^{\alpha}$ into $\psi \partial_{s^{\prime}} w_{0}^{\alpha}+(1-\psi) \partial_{s^{\prime}} w_{0}^{\alpha}$. Then, noting that $\left(\partial_{t}^{2}-\rho_{0}^{-1} \mathcal{A}_{0}\right) w_{+}^{\beta}(t, x ; \omega)=0$ in $\mathbb{R} \times(\Omega \cap \operatorname{supp}[\psi])$, we have by integration by parts

$$
\begin{gathered}
\int_{\Omega \cap \mathbb{R}_{+}^{3}} \int_{\mathbb{R}}\left(\partial_{s^{\prime}}^{2}-\rho_{0}^{-1} \mathcal{A}_{0}\right) w_{+}^{\beta}\left(s^{\prime}-s, y ; \omega\right) \cdot \partial_{s^{\prime}} w_{0}^{\alpha}\left(s^{\prime}, y ; \theta\right) d s^{\prime} \rho_{0} d y \\
=\int_{\Omega \cap \mathbb{R}_{+}^{3}} \int_{\mathbb{R}} w_{+}^{\beta}\left(s^{\prime}-s, y ; \omega\right) \cdot\left(\partial_{s^{\prime}}^{2}-\rho_{0}^{-1} \mathcal{A}_{0}\right)\left((1-\psi) \partial_{s^{\prime}} w_{0}^{\alpha}\right)\left(s^{\prime}, y ; \theta\right) d s^{\prime} \rho_{0} d y \\
-\int_{\partial\left(\Omega \cap \mathbb{R}_{+}^{3}\right)}\left\{\int_{\mathbb{R}}\left(\tilde{\mathcal{N}}_{0} w_{+}^{\beta}\right)\left(s^{\prime}-s, y ; \omega\right) \cdot(1-\psi) \partial_{s^{\prime}} w_{0}^{\alpha}\left(s^{\prime}, y ; \theta\right) d s^{\prime}\right. \\
\left.-\int_{\mathbb{R}} w_{+}^{\beta}\left(s^{\prime}-s, y ; \omega\right) \cdot \tilde{\mathcal{N}}_{0}\left((1-\psi) \partial_{s^{\prime}} w_{0}^{\alpha}\right)\left(s^{\prime}, y ; \theta\right) d s^{\prime}\right\} d S_{y} .
\end{gathered}
$$

Here, we note that the integration in $s^{\prime}$ (in (2.3)) is well-defined and that the integration by parts is valid. These verifications are reduced to examination of the decay properties of $w_{+}^{\beta}$ and $w_{0}^{\alpha}$ as $s^{\prime} \rightarrow \pm \infty$ (cf. section 2 and 6 of [8]). Combining (2.3) and Theorem 2.2, we have

$$
\begin{aligned}
\tilde{S}_{\alpha \beta}(s, \theta, \omega)=- & \int_{\Omega \cap \mathbb{R}_{+}^{3}} \int_{\mathbb{R}} w_{+}^{\beta}\left(s^{\prime}-s, y ; \omega\right) \cdot\left(\partial_{s^{\prime}}^{2}-\rho_{0}^{-1} \mathcal{A}_{0}\right)\left(\psi \partial_{s^{\prime}} w_{0}^{\alpha}\right)\left(s^{\prime}, y ; \theta\right) d s^{\prime} \rho_{0} d y \\
& +\int_{\partial\left(\Omega \cap \mathbb{R}_{+}^{3}\right)} \int_{\mathbb{R}}\left\{\left(\tilde{\mathcal{N}}_{0} w_{+}^{\beta}\right)\left(s^{\prime}-s, y ; \omega\right) \cdot\left(\psi \partial_{s^{\prime}} w_{0}^{\alpha}\right)\left(s^{\prime}, y ; \theta\right) d s^{\prime}\right. \\
& \left.-w_{+}^{\beta}\left(s^{\prime}-s, y ; \omega\right) \cdot \tilde{\mathcal{N}}_{0}\left(\psi \partial_{s^{\prime}} w_{0}^{\alpha}\right)\left(s^{\prime}, y ; \theta\right) d s^{\prime}\right\} d S_{y}
\end{aligned}
$$


where we use $\left(\partial_{t}^{2}-\rho_{0}^{-1} \mathcal{A}_{0}\right) w_{0}^{\alpha}(t, x ; \theta)=0$ in $\mathbb{R} \times \mathbb{R}_{+}^{3}$.

Using $\left(\partial_{t}^{2}-\rho_{0}^{-1} \mathcal{A}_{0}\right) w_{0}^{\alpha}(t, x ; \theta)=0$ again and noting that $\operatorname{supp}[1-\psi(y)]$ is bounded, we obtain the following in the same way as in (2.3):

$$
\begin{gathered}
0=\int_{\Omega \cap \mathbb{R}_{+}^{3}} \int_{\mathbb{R}}\left(\partial_{s^{\prime}}^{2}-\rho_{0}^{-1} \mathcal{A}_{0}\right) w_{0}^{\beta}\left(s^{\prime}-s, y ; \omega\right) \cdot(1-\psi(y)) \partial_{s^{\prime}} w_{0}^{\alpha}\left(s^{\prime}, y ; \theta\right) d s^{\prime} \rho_{0} d y \\
=-\int_{\Omega \cap \mathbb{R}_{+}^{3}} \int_{\mathbb{R}} w_{0}^{\beta}\left(s^{\prime}-s, y ; \omega\right) \cdot\left(\partial_{s^{\prime}}^{2}-\rho_{0}^{-1} \mathcal{A}_{0}\right)\left(\psi \partial_{s^{\prime}} w_{0}^{\alpha}\right)\left(s^{\prime}, y ; \theta\right) d s^{\prime} \rho_{0} d y \\
-\int_{\partial\left(\Omega \cap \mathbb{R}_{+}^{3}\right)} \int_{\mathbb{R}}\left\{\tilde{\mathcal{N}}_{0} w_{0}^{\beta}\left(s^{\prime}-s, y ; \omega\right) \cdot(1-\psi) \partial_{s^{\prime}} w_{0}^{\alpha}\left(s^{\prime}, y ; \theta\right)\right. \\
\left.-w_{0}^{\beta}\left(s^{\prime}-s, y ; \omega\right) \cdot \tilde{\mathcal{N}}_{0}\left((1-\psi) \partial_{s^{\prime}} w_{0}^{\alpha}\right)\left(s^{\prime}, y ; \theta\right)\right\} d s^{\prime} d S_{y} .
\end{gathered}
$$

Summing each side of the above equality and each of (2.4) and noting supp $[\psi] \subset B_{r_{0}}^{c}$ $\left(=\mathbb{R}^{3} \backslash B_{r_{0}}\right)$, we have

$$
\begin{aligned}
\tilde{S}_{\alpha \beta}(s, \theta, \omega) & \\
= & -\int_{\Omega} \int_{\mathbb{R}}\left(w_{0}^{\beta}+w_{+}^{\beta}\right)\left(s^{\prime}-s, y ; \omega\right) \cdot\left(\partial_{s^{\prime}}^{2}-\rho_{0}^{-1} \mathcal{A}_{0}\right)\left(\psi \partial_{s^{\prime}} w_{0}^{\alpha}\right)\left(s^{\prime}, y ; \theta\right) d s^{\prime} \rho_{0} d y \\
& +\int_{\Gamma} \int_{\mathbb{R}}\left\{\mathcal{N}\left(w_{0}^{\beta}+w_{+}^{\beta}\right)\left(s^{\prime}-s, y ; \omega\right) \cdot\left(\psi \partial_{s^{\prime}} w_{0}^{\alpha}\right)\left(s^{\prime}, y ; \theta\right)\right. \\
& -\int_{\partial\left(\Omega \cap \mathbb{R}_{+}^{3}\right)} \int_{\mathbb{R}}\left\{\tilde{\mathcal{N}}_{0} w_{0}^{\beta}\left(s^{\prime}-s, y ; \omega\right) \cdot \partial_{s^{\prime}} w_{0}^{\alpha}\left(s^{\prime}, y ; \theta\right)\right. \\
= & -\int_{\Omega} \int_{\mathbb{R}} w_{+, t o t}^{\beta}\left(s^{\prime}-s, y ; \omega\right) \cdot\left(\partial_{s^{\prime}}^{2}-\rho_{0}^{-1} \mathcal{A}_{0}\right)\left(\psi \partial_{s^{\prime}} w_{0}^{\alpha}\right)\left(s^{\prime}, y ; \theta\right) d s^{\prime} \rho_{0} d y \\
& -\int_{\Gamma} \int_{\mathbb{R}} w_{+, t o t}^{\beta}\left(s^{\prime}-s, y ; \omega\right) \cdot \mathcal{N}_{0}\left(\psi \partial_{s^{\prime}} w_{0}^{\alpha}\right)\left(s^{\prime}, y ; \theta\right) d s^{\prime} d S_{y} \\
& -\int_{\Gamma \cap \mathbb{R}_{+}^{3}} \int_{\mathbb{R}}\left\{\left(\tilde{\mathcal{N}}_{0} w_{0}^{\beta}\right)\left(s^{\prime}-s, y ; \omega\right) \cdot \partial_{s^{\prime}} w_{0}^{\alpha}\left(s^{\prime}, y ; \theta\right)\right. \\
& \left.-w_{0}^{\beta}\left(s^{\prime}-s, y ; \omega\right) \cdot \tilde{\mathcal{N}}_{0} \partial_{s^{\prime}} w_{0}^{\alpha}\left(s^{\prime}, y ; \theta\right) d s^{\prime}\right\} d S_{y} .
\end{aligned}
$$

Here, note that $\partial\left(\Omega \cap \mathbb{R}_{+}^{3}\right)=\left(\bar{\Omega} \cap \partial \mathbb{R}_{+}^{3}\right) \cup\left(\Gamma \cap \mathbb{R}_{+}^{3}\right)$ and that $\tilde{\mathcal{N}}_{0} w_{0}^{\gamma}=0$ on $\mathbb{R} \times \partial \mathbb{R}_{+}^{3}$. The last integral $-\int_{\Gamma \cap \mathbb{R}_{+}^{3}} \int_{\mathbb{R}}\{\cdots\} d s^{\prime} d S_{y}$ is equal to 0 since $\bar{\Omega}^{c} \cap \mathbb{R}_{+}^{3}$ is bounded and

$$
\begin{aligned}
& 0= \int_{\bar{\Omega}^{c} \cap \mathbb{R}_{+}^{3}} \int_{\mathbb{R}^{\prime}}\left(\partial_{s^{\prime}}^{2}-\rho_{0}^{-1} \mathcal{A}_{0}\right) w_{0}^{\beta}\left(s^{\prime}-s, y ; \omega\right) \cdot \partial_{s^{\prime}} w_{0}^{\alpha}\left(s^{\prime}, y ; \theta\right) d s^{\prime} \rho_{0} d y \\
&= \int_{\bar{\Omega}^{c} \cap \mathbb{R}_{+}^{3}} \int_{\mathbb{R}} w_{0}^{\beta}\left(s^{\prime}-s, y ; \omega\right) \cdot\left(\partial_{s^{\prime}}^{2}-\rho_{0}^{-1} \mathcal{A}_{0}\right) \partial_{s^{\prime}} w_{0}^{\alpha}\left(s^{\prime}, y ; \theta\right) d s^{\prime} \rho_{0} d y \\
&-\int_{\partial\left(\bar{\Omega}^{c} \cap \mathbb{R}_{+}^{3}\right)} \int_{\mathbb{R}}\left\{\tilde{\mathcal{N}}_{0} w_{0}^{\beta}\left(s^{\prime}-s, y ; \omega\right) \cdot \partial_{s^{\prime}} w_{0}^{\alpha}\left(s^{\prime}, y ; \theta\right)\right. \\
&=-\int_{\Gamma \cap \mathbb{R}_{+}^{3}} \int_{\mathbb{R}}\left\{\tilde{\mathcal{N}}_{0} w_{0}^{\beta}\left(s^{\prime}-s, y ; \omega\right) \cdot \partial_{s^{\prime}} w_{0}^{\alpha}\left(s^{\prime}, y ; \theta\right)\right. \\
&\left.\quad-w_{0}^{\beta}\left(s^{\prime}-s, y ; \omega\right) \cdot \tilde{\mathcal{N}}_{0} \partial_{s^{\prime}} w_{0}^{\alpha}\left(s^{\prime}, y ; \theta\right)\right\} d s^{\prime} d S_{y},
\end{aligned}
$$

where we use $\partial\left(\bar{\Omega}^{c} \cap \mathbb{R}_{+}^{3}\right)=\left(\bar{\Omega}^{c} \cap \partial \mathbb{R}_{+}^{3}\right) \cup\left(\Gamma \cap \mathbb{R}_{+}^{3}\right)$. Thus Proposition 2.3 is proved. 
From here, we reduce the expression of the kernel stated in Proposition 2.3 to the form containing only one integral as shown in Theorem 3.1. The idea of this reduction is as follows. We introduce the incoming solution $w(t, x)$ of the perturbed equation

$$
\begin{cases}\left(\partial_{t}^{2}-\rho^{-1} \mathcal{A}\right) w(t, x)=q^{\alpha}(t, x ; \theta) & \text { in } \mathbb{R} \times \Omega, \\ \mathcal{N} w(t, x)=m^{\alpha}(t, x ; \theta) & \text { on } \mathbb{R} \times \Gamma\end{cases}
$$

where $q^{\alpha}(t, x ; \theta)=\left(\partial_{t}^{2}-\rho_{0}^{-1} \mathcal{A}_{0}\right)\left(\psi w_{0}^{\alpha}\right)(t, x ; \theta)$ and $m^{\alpha}(t, x ; \theta)=\mathcal{N}_{0}\left(\psi w_{0}^{\alpha}\right)(t, x ; \theta)$. Using $w(t, x)$, we substitute the operators $\left(\partial_{t}^{2}-\rho^{-1} \mathcal{A}\right)$ and $\mathcal{N}$ for $\left(\partial_{t}^{2}-\rho_{0}^{-1} \mathcal{A}_{0}\right)$ and $\mathcal{N}_{0}$ in the expression of the kernel in Proposition 2.3. Then, we can get the required expression through integration by parts. However, it is difficult to carry out this procedure exactly. We consider the product $\langle\tilde{S}(\cdot, \omega, \theta), \varphi\rangle$ in the distribution sense for the localized test functions $\varphi(s) \in C_{0}^{\infty}(I)$, where $I$ is a finite open interval for the localization. Then, taking the integrals of convolution type between the data $q^{\alpha}$ and $m^{\alpha}$ with the test function $\varphi$, we can make an (approximate) solution concretely to substitute $\left(\partial_{t}^{2}-\rho^{-1} \mathcal{A}\right)$ and $\mathcal{N}$ for $\left(\partial_{t}^{2}-\rho_{0}^{-1} \mathcal{A}_{0}\right)$ and $\mathcal{N}_{0}$. We examine properties of $\tilde{S}(\cdot, \omega, \theta)$ as the functional: $\varphi \mapsto\langle\tilde{S}(\cdot, \omega, \theta), \varphi\rangle$. Note that the distribution $J$ belongs

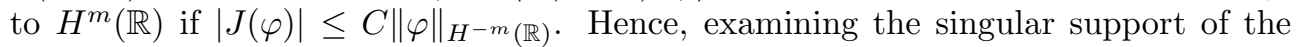
distributions in $I$, we can neglect those terms which are estimated in the following definition.

DEFINITION 2.4. Let $J$ be a linear functional (mapping) from $C_{0}^{\infty}(I)$ to the Banach space $X$ with the norm $\|\cdot\|_{X}$. We say that the functional $J$ is negligible when for any positive integer $N$

$$
\|J(\varphi)\|_{X} \leq C_{N}\|\varphi\|_{H^{-N}(\mathbb{R})}, \quad \varphi \in C_{0}^{\infty}(I)
$$

Furthermore, fixing $I$, we write $J \simeq K$ when $J-K$ is negligible; if a more precise description is necessary, we write " $J \simeq K$ with respect to $C_{0}^{\infty}(I) \rightarrow X$ ".

Let $s_{0} \in \mathbb{R}$ and fix $I=\left(s_{0}-\varepsilon, s_{0}+\varepsilon\right)$ for a sufficiently small constant $\varepsilon>0$. For any $\varphi \in C_{0}^{\infty}(I)$ we have by Proposition 2.3

$$
\begin{aligned}
\left\langle\tilde{S}_{\alpha \beta}(\cdot, \theta, \omega), \varphi\right\rangle & =-\int_{\Omega} \int_{\mathbb{R}} w_{+, t o t}^{\beta}\left(s^{\prime}, y ; \omega\right) \cdot \varphi \star\left(\partial_{s} q^{\alpha}\right)\left(s^{\prime}, y ; \theta\right) d s^{\prime} \rho_{0} d y \\
& -\int_{\Gamma} \int_{\mathbb{R}} w_{+, t o t}^{\beta}\left(s^{\prime}, y ; \omega\right) \cdot \varphi \star\left(\partial_{s} m^{\alpha}\right)\left(s^{\prime}, y ; \theta\right) d s^{\prime} d S_{y}
\end{aligned}
$$

where $\star$ means $f \star g(t)=\int_{\mathbb{R}} f(s) g(s+t) d s$, which is connected with the convolution $*$ as $f \star g(t)=f *(g(-\cdot))(-t)$. We can check that $\varphi \star\left(\partial_{s} q^{\alpha}\right)(s, y ; \theta)$ and $\varphi \star\left(\partial_{s} m^{\alpha}\right)(s, y ; \theta)$ are $C^{\infty}$ functions and that the above integrals in $s^{\prime}$ are well defined. Note that $\partial_{s} q^{\alpha}(\cdot, y ; \theta)$ and $\partial_{s} m^{\alpha}(\cdot, y ; \theta)$ have singularities of the type of the Dirac $\delta$-function (for the precise form, see section 5 of [8]), and that

$$
\begin{aligned}
& \operatorname{supp}\left[q^{\alpha}(\cdot, \cdot ; \theta)\right] \subset \mathbb{R} \times\left(\Omega \cap \mathbb{R}_{+}^{3} \cap\left(B_{r_{0}+2} \backslash B_{r_{0}+1}\right)\right) \\
& \operatorname{supp}\left[m^{\alpha}(\cdot, \cdot ; \theta)\right] \subset \mathbb{R} \times\left(\Gamma \cap \partial \mathbb{R}_{+}^{3} \cap\left(B_{r_{0}+2} \backslash B_{r_{0}+1}\right)\right) \\
& \operatorname{sing} \operatorname{supp}\left[q^{\alpha}(\cdot, \cdot ; \theta)\right] \subset\left\{(s, y) \in \mathbb{R} \times \Omega ;|s| \leq c_{R}^{-1}\left(r_{0}+2\right), r_{0}+1 \leq|y| \leq r_{0}+2\right\} \\
& \operatorname{sing} \operatorname{supp}\left[m^{\alpha}(\cdot, \cdot ; \theta)\right] \subset\left\{(s, y) \in \mathbb{R} \times \Gamma ;|s| \leq c_{R}^{-1}\left(r_{0}+2\right), r_{0}+1 \leq|y| \leq r_{0}+2\right\}
\end{aligned}
$$

We choose $t_{1}>\max \left\{c_{R}^{-1}\left(r_{0}+5\right)+3 \varepsilon,-s_{0}+c_{R}^{-1}\left(r_{0}+5\right)\right\}$ and a cutoff function $\phi_{1} \in C_{0}^{\infty}(\mathbb{R})$ with $0 \leq \phi_{1} \leq 1, \phi_{1}(t)=1$ if $\left|t+s_{0}\right| \leq t_{1}+c_{R}^{-1}, \phi_{1}(t)=0$ if 
$\left|t+s_{0}\right| \geq t_{1}+2 c_{R}^{-1}$, and put

$$
\begin{aligned}
J_{1}(\varphi) & =-\int_{\Omega} \int_{\mathbb{R}} \phi_{1}\left(s^{\prime}\right) w_{+, t o t}^{\beta}\left(s^{\prime}, y ; \omega\right) \cdot \varphi \star\left(\partial_{s} q^{\alpha}\right)\left(s^{\prime}, y ; \theta\right) d s^{\prime} \rho_{0} d y \\
& -\int_{\Gamma} \int_{\mathbb{R}} \phi_{1}\left(s^{\prime}\right) w_{+, t o t}^{\beta}\left(s^{\prime}, y ; \omega\right) \cdot \varphi \star\left(\partial_{s} m^{\alpha}\right)\left(s^{\prime}, y ; \theta\right) d s^{\prime} d S_{y} .
\end{aligned}
$$

Then, we have $J_{1}(\varphi) \simeq\left\langle\tilde{S}_{\alpha \beta}(\cdot, \theta, \omega), \varphi\right\rangle$, i.e., $J_{1}-\tilde{S}_{\alpha \beta}(\cdot, \theta, \omega)$ is negligible in the sense of Definition 2.4 with $X=\mathbb{C}$. This follows from (2.5) and the following estimates for any $N, N^{\prime} \in \mathbb{N}$ and $\delta<1 / 2$ :

$$
\left\{\begin{aligned}
\left\|\left(1-\phi_{1}(\cdot)\right)(1+|\cdot|)^{1+\delta} \varphi \star\left(\partial_{s} q^{\alpha}\right)(\cdot, y ; \theta)\right\|_{H^{N}(\mathbb{R})} \leq C_{N, N^{\prime}}\|\varphi\|_{H^{-N^{\prime}}(\mathbb{R})} & \text { for any } y \in \Omega, \varphi \in C_{0}^{\infty}(I) \\
\left\|\left(1-\phi_{1}(\cdot)\right)(1+|\cdot|)^{1+\delta} \varphi \star\left(\partial_{s} m^{\alpha}\right)(\cdot, y ; \theta)\right\|_{H^{N}(\mathbb{R})} \leq C_{N, N^{\prime}}\|\varphi\|_{H^{-N^{\prime}}(\mathbb{R})} & \text { for any } y \in \Gamma, \varphi \in C_{0}^{\infty}(I)
\end{aligned}\right.
$$

These estimates are derived from the form of $w_{0}^{\alpha}(s, y ; \theta)$ (cf. section 5 of [8]).

To estimate $J_{1}(\varphi)$, we have only to take account of the integrals in $s^{\prime}$ on the interval $I_{t_{1}+3 c_{R}^{-1}}\left(s_{0}\right)=\left[-s_{0}-\left(t_{1}+3 c_{R}^{-1}\right),-s_{0}+\left(t_{1}+3 c_{R}^{-1}\right)\right]$. We construct an approximate solution of the following equation in the later sections (sections 4 and 5 ):

$$
\begin{cases}\left(\partial_{t}^{2}-\rho^{-1} \mathcal{A}\right) v^{\alpha}(t, x ; \theta)=\varphi \star\left(\partial_{t} q^{\alpha}\right)(t, x ; \theta) & \text { in } I_{t_{1}+3 c_{R}^{-1}}\left(s_{0}\right) \times \Omega \\ \mathcal{N} v^{\alpha}(t, x ; \theta)=\varphi \star\left(\partial_{t} m^{\alpha}\right)(t, x ; \theta) & \text { on } I_{t_{1}+3 c_{R}^{-1}}\left(s_{0}\right) \times \Gamma\end{cases}
$$

More precisely, the approximate solution $v_{\varphi}^{\alpha}(t, x ; \theta)$ of this equation has the properties (i) and (ii):

(i) There exists a constant $l_{0} \in \mathbb{R}$ such that for any $\eta \in C_{0}^{\infty}\left(I_{t_{1}+3 c_{R}^{-1}}\left(s_{0}\right)\right)$ and integers $m \in \mathbb{Z}, j, N(\geq 0)$, we have

$$
\max _{|\gamma| \leq N} \sup _{x \in \bar{\Omega}}\left\|\eta(\cdot) \partial_{s}^{j} \partial_{x}^{\gamma} v_{\varphi}^{\alpha}(\cdot, x ; \theta)\right\|_{H^{m}(\mathbb{R})} \leq C_{\eta, m, j, N}\|\varphi\|_{H^{m+N+l_{0}+j}(\mathbb{R})}, \quad\left(\varphi \in C_{0}^{\infty}(I)\right) .
$$

(ii) For any positive integer $N$ the function $v_{\varphi}^{\alpha}(t, x ; \theta)$ satisfies

$$
\left\{\begin{aligned}
&\left(\partial_{t}^{2}-\rho^{-1} \mathcal{A}\right) v_{\varphi}^{\alpha}(t, x ; \theta) \simeq \varphi \star\left(\partial_{t} q^{\alpha}\right)(t, x ; \theta) \\
& \text { with respect to } C_{0}^{\infty}(I) \rightarrow \mathcal{B}^{N}\left(\overline{I_{t_{1}+3 c_{R}^{-1}}\left(s_{0}\right) \times \Omega}\right), \\
& \mathcal{N}\left(x, \partial_{x}\right) v_{\varphi}^{\alpha}(t, x ; \theta) \simeq \varphi \star\left(\partial_{t} m^{\alpha}\right)(t, x ; \theta) \quad \text { with respect to } C_{0}^{\infty}(I) \rightarrow \mathcal{B}^{N}\left(\overline{I_{t_{1}+3 c_{R}^{-1}}\left(s_{0}\right) \times \Gamma}\right), \\
& v_{\varphi}^{\alpha}(t, x ; \theta)=0, \text { if }|x| \geq r_{1}\left(=r_{0}+2+c_{P}\left(t_{1}+3 c_{R}^{-1}\right)\right) \\
& v_{\varphi}^{\alpha} \simeq 0 \quad \text { with respect to } C_{0}^{\infty}(I) \rightarrow \mathcal{B}^{N}\left(\left[-s_{0}+c_{R}^{-1}\left(r_{0}+3\right)+2 \varepsilon, \infty\right) \times \bar{\Omega}\right),
\end{aligned}\right.
$$

where $\mathcal{B}^{N}(D)$ is the set consisting of $N$-th order differentiable functions satisfying all derivatives are continuous and bounded on $D$.

Hereafter (in this and next sections), we assume that the above $v_{\varphi}^{\alpha}$ exists. For $J_{1}(\varphi)$, using $v_{\varphi}^{\alpha}$, we substitute $\left(\partial_{t}^{2}-\rho^{-1} \mathcal{A}\right) \partial_{t} v_{\varphi}^{\alpha}$ and $\mathcal{N} \partial_{t} v_{\varphi}^{\alpha}$ for $\varphi \star\left(\partial_{t} q^{\alpha}\right)\left(=\varphi \star\left(\partial_{t}^{2}-\right.\right.$ $\left.\left.\rho_{0}^{-1} \mathcal{A}_{0}\right) \partial_{t}\left(\psi w_{0}^{\alpha}\right)\right)$ and $\varphi \star\left(\partial_{t} m^{\alpha}\right)\left(=\varphi \star \partial_{t} \mathcal{N}_{0}\left(\psi w_{0}^{\alpha}\right)\right)$ respectively. Then, noting that the commutator $\partial_{s}^{2}\left(\phi_{1} \cdot\right)-\phi_{1}\left(\partial_{s}^{2} \cdot\right)$ is of the form $\partial_{s}^{2} \phi_{1}+2 \partial_{s} \phi_{1} \partial_{s}$ and that support of $q^{\alpha}$ and $m^{\alpha}$ is estimated in (2.5), we see by (2.9) and integration by parts that

$$
J_{1}(\varphi) \simeq-\int_{\Omega} \int_{\mathbb{R}} \rho(y)\left(\partial_{s}^{2} \phi_{1}(s)+2 \partial_{s} \phi_{1}(s) \partial_{s}\right) w_{+, t o t}^{\beta}(s, y ; \omega) \cdot v_{\varphi}^{\alpha}(s, y ; \theta) d s d y .
$$


Applying a further reduction to the right side of (2.10), we obtain the required form of the functional $\left\langle\tilde{S}_{\alpha \beta}(\cdot, \theta, \omega), \varphi\right\rangle$ :

Proposition 2.5. Let $\phi_{2}(s)$ be a $C^{\infty}$ cutoff function such that $0 \leq \phi_{2} \leq 1$, $\phi_{2}(s)=1$ for $s<-s_{0}-c_{R}^{-1}$ and $\phi_{2}(s)=0$ for $s>-s_{0}+c_{R}^{-1}$, and set

$$
\begin{aligned}
J_{2}(\varphi)=-\int_{\Omega} \int_{\mathbb{R}} \phi_{2}(s) \rho(y) \partial_{s} \phi_{1}(s) \psi(y)\left\{\partial_{s} w_{0}^{\beta}(s, y ; \omega) \cdot v_{\varphi}^{\alpha}(s, y ; \theta)\right. \\
\left.-w_{0}^{\beta}(s, y ; \omega) \cdot \partial_{s} v_{\varphi}^{\alpha}(s, y ; \theta)\right\} d s d y .
\end{aligned}
$$

Then, $J_{2}(\varphi)-\left\langle\tilde{S}_{\alpha \beta}(\cdot, \theta, \omega), \varphi\right\rangle$ is negligible, i.e.,

$$
\left\langle\tilde{S}_{\alpha \beta}(\cdot, \theta, \omega), \varphi\right\rangle \simeq J_{2}(\varphi) \text { with respect to } C_{0}^{\infty}(I) \rightarrow \mathbb{C} .
$$

Proof. We see from (2.9) that $\int_{\Omega} \int_{\mathbb{R}}\left(1-\phi_{2}\right) \rho\left(\partial_{s}^{2} \phi_{1}+2\left(\partial_{s} \phi_{1}\right) \partial_{s}\right) w_{+, t o t}^{\beta} \cdot v_{\varphi}^{\alpha} d s d y$ $\simeq 0$, which implies that $J_{1}(\varphi)$ in $(2.10)\left(\simeq\left\langle\tilde{S}_{\alpha \beta}(\cdot, \theta, \omega), \varphi\right\rangle\right)$ satisfies

$$
J_{1}(\varphi) \simeq-\int_{\Omega} \int_{\mathbb{R}} \phi_{2}(s) \rho(y)\left(\partial_{s}^{2} \phi_{1}(s)+2 \partial_{s} \phi_{1}(s) \partial_{s}\right) w_{+, t o t}^{\beta}(s, y ; \omega) \cdot v_{\varphi}^{\alpha}(s, y ; \omega) d s d y .
$$

It follows from $\partial_{s} \phi_{2} \partial_{s} \phi_{1}=0$ that $\phi_{2}\left(\partial_{s}^{2} \phi_{1}\right) w_{+, t o t}^{\beta}=\partial_{s}\left(\phi_{2}\left(\partial_{s} \phi_{1}\right) w_{+, t o t}^{\beta}\right)-$ $\phi_{2} \partial_{s} \phi_{1} \partial_{s} w_{+, t o t}^{\beta}$. Hence, using integration by parts, we obtain

$$
\begin{aligned}
J_{1}(\varphi) \simeq-\int_{\Omega} \int_{\mathbb{R}} \phi_{2}(s) \rho(y) \partial_{s} \phi_{1}(s)\left\{\partial_{s} w_{+, t o t}^{\beta}(s, y ; \omega) \cdot v_{\varphi}^{\alpha}(s, y ; \theta)\right. \\
\left.-w_{+, t o t}^{\beta}(s, y ; \omega) \cdot \partial_{s} v_{\varphi}^{\alpha}(s, y ; \theta)\right\} d s d y .
\end{aligned}
$$

Take a $C^{\infty}$ cutoff function $\phi_{3}(t)$ such that $\phi_{3}(t)=1$ for $t<-c_{R}^{-1}\left(r_{0}+5\right)$ and $\phi_{3}(t)=0$ for $t>-c_{R}^{-1}\left(r_{0}+4\right)$, and set

$$
\begin{array}{rlrl}
q_{1}^{\beta}(t, x ; \omega) & =\left(1-\phi_{3}(t)\right) q^{\beta}(t, x ; \omega), & q_{2}^{\beta}(t, x ; \omega) & =\phi_{3}(t) q^{\beta}(t, x ; \omega), \\
m_{1}^{\beta}(t, x ; \omega) & =\left(1-\phi_{3}(t)\right) m^{\beta}(t, x ; \omega), \quad m_{2}^{\beta}(t, x ; \omega)=\phi_{3}(t) m^{\beta}(t, x ; \omega) .
\end{array}
$$

We employ the solutions of the equation

$$
\begin{cases}\left(\partial_{t}^{2}-\rho^{-1} \mathcal{A}\right) \tilde{w}_{+, j}^{\beta}(t, x ; \omega)=-q_{j}^{\beta}(t, x ; \omega) & \text { in } \mathbb{R} \times \Omega, \\ \mathcal{N} \tilde{w}_{+, j}^{\beta}(t, x ; \omega)=-m_{j}^{\beta}(t, x ; \omega) & \text { on } \mathbb{R} \times \Gamma, \\ \tilde{w}_{+, j}^{\beta}(t, x ; \omega) \text { satisfies }(+) \text {-condition. } & \end{cases}
$$

Then, we can decompose $w_{+, t o t}^{\beta}$ in the form $w_{+, t o t}^{\beta}=\psi w_{0}^{\beta}+\tilde{w}_{+, 1}^{\beta}+\tilde{w}_{+, 2}^{\beta}$, and by (2.5) have $\tilde{w}_{+, 1}^{\beta}(t, x ; \omega)=0$ for $t<-c_{R}^{-1}\left(r_{0}+5\right)$ and $\tilde{w}_{+, 2}^{\beta}(t, x ; \omega) \in C^{\infty}(\mathbb{R} \times \bar{\Omega})$. Therefore, noting that supp $\left[\phi_{2} \partial_{s} \phi_{1}\right] \subset\left(-s_{0}-t_{1}-2 c_{R}^{-1},-s_{0}-t_{1}-c_{R}^{-1}\right) \subset\left(-s_{0}-\right.$ $\left.t_{1}-2 c_{R}^{-1},-c_{R}^{-1}\left(r_{0}+6\right)\right)$ and $\operatorname{supp}\left[\nabla_{x} \psi\right] \subset B_{r_{0}+2} \backslash B_{r_{0}+1}$, we see that

$$
\begin{aligned}
& w_{+, t o t}^{\beta}(t, x ; \omega)-\psi(x) w_{0}^{\beta}(t, x ; \omega) \text { and }\left(\nabla_{x} \psi\right) w_{0}^{\beta}(t, x ; \omega) \text { are } \\
& C^{\infty} \text { for } t \text { in a neighborhood of supp }\left[\phi_{2} \partial_{s} \phi_{1}\right] \text { and } x \in \bar{\Omega} .
\end{aligned}
$$

From this fact, it follows that $h_{+,-\infty}^{\beta}(s, x ; \omega)=w_{+, t o t}^{\beta}(s, x ; \omega)-\psi(x) w_{0}^{\beta}(s, x ; \omega)$ satisfies $\phi_{2} \partial_{s} \phi_{1} h_{+,-\infty}^{\beta} \in C^{\infty}(\mathbb{R} \times \bar{\Omega})$. On the other hand we have

$$
\begin{array}{r}
J_{1}(\varphi) \simeq J_{2}(\varphi)-\int_{\Omega} \int_{\mathbb{R}} \phi_{2}(s) \rho(y) \partial_{s} \phi_{1}(s)\left\{\partial_{s} h_{+,-\infty}^{\beta}(s, y ; \omega) \cdot v_{\varphi}^{\alpha}(s, y ; \theta)\right. \\
\left.-h_{+,-\infty}^{\beta}(s, y ; \omega) \cdot \partial_{s} v_{\varphi}^{\alpha}(s, y ; \theta)\right\} d s d y .
\end{array}
$$


We can verify that the second term of the above right side is negligible if we show the following lemma. Thus, Proposition 2.5 is proved.

LEMma 2.6. Let $h(s, y) \in C^{\infty}(\mathbb{R} \times \Omega)$ and $\eta(s) \in C_{0}^{\infty}\left(I_{t_{1}+3 c_{R}^{-1}}\left(s_{0}\right)\right)$, and set

$$
J_{-\infty}(\varphi)=\int_{\Omega} \int_{\mathbb{R}} \eta(s) h(s, y) \cdot v_{\varphi}^{\alpha}(s, y ; \theta) d s d y, \quad \varphi \in C_{0}^{\infty}(I) .
$$

Then, $J_{-\infty}(\varphi)$ is negligible, i.e., $J_{-\infty}(\varphi) \simeq 0$ with respect to $C_{0}^{\infty}(I) \rightarrow \mathbb{C}$.

Proof. We take a cutoff function $\tilde{\eta}(s) \in C_{0}^{\infty}\left(I_{t_{1}+3 c_{R}^{-1}}\left(s_{0}\right)\right)$ with $\tilde{\eta}(s)=1$ near $\operatorname{supp}[\eta]$. Since $\operatorname{supp}\left[v_{\varphi}^{\alpha}(\cdot, \cdot ; \theta)\right] \subset \overline{I_{t_{1}+3 c_{R}^{-1}}\left(s_{0}\right)} \times\left(\overline{\Omega \cap B_{r_{1}}}\right)$, we have

$$
\begin{aligned}
& \left|J_{-\infty}(\varphi)\right|=\left|\int_{\Omega} \int_{\mathbb{R}} \eta(s) h(s, y) \cdot \tilde{\eta}(s) v_{\varphi}^{\alpha}(s, y ; \theta) d s d y\right| \\
& \quad=\left|\int_{\Omega} \int_{\mathbb{R}}\left\langle D_{s}\right\rangle^{N+l_{0}}(\eta(s) h(s, y)) \cdot\left\langle D_{s}\right\rangle^{-\left(N+l_{0}\right)}\left(\tilde{\eta}(s) v_{\varphi}^{\alpha}(s, y ; \theta)\right) d s d y\right| \\
& \quad \leq\left|B_{r_{1}}\right| \sup _{x \in \overline{\Omega \cap B_{r_{1}}}}\left\{\left\|\left\langle D_{s}\right\rangle^{N+l_{0}}(\eta(\cdot) h(\cdot, x))\right\|_{L^{2}(\mathbb{R})}\left\|\left\langle D_{s}\right\rangle^{-\left(N+l_{0}\right)}\left(\tilde{\eta}(\cdot) v_{\varphi}^{\alpha}(\cdot, x ; \theta)\right)\right\|_{L^{2}(\mathbb{R})}\right\},
\end{aligned}
$$

where $\langle s\rangle=\left(1+|s|^{2}\right)^{1 / 2},\left|B_{r_{1}}\right|$ is the volume of $B_{r_{1}}$ and $l_{0}$ is the number in (2.8). From this estimate and (2.8), it follows that

$$
\begin{aligned}
\left|J_{-\infty}(\varphi)\right| & \leq\left|B_{r_{1}}\right| \sup _{x \in \overline{\Omega \cap B_{r_{1}}}}\left\{\|\eta(\cdot) h(\cdot, x)\|_{H^{N+l_{0}(\mathbb{R})}}\left\|\tilde{\eta}(\cdot) v_{\varphi}^{\alpha}(\cdot, x ; \theta)\right\|_{H^{-\left(N+l_{0}\right)}(\mathbb{R})}\right\} \\
& \leq\left|B_{r_{1}}\right| C_{N} \sup _{x \in \overline{\Omega \cap B_{r_{1}}}}\|\eta(\cdot) h(\cdot, x)\|_{H^{N+l_{0}}(\mathbb{R})}\|\varphi\|_{H^{-N}(\mathbb{R})} .
\end{aligned}
$$

This completes the proof of Lemma 2.6.

3. Reduction for the channel of the Rayleigh wave. The Rayleigh wave is concentrated on the boundary $\Gamma$, i.e., it is $C^{\infty}$ inside the domain $\Omega$ and decays in distance from $\Gamma$ even if it has singularities on $\Gamma$. Furthermore, the hyperbolicity of the Rayleigh wave seems to be characterized by the terms of the Neumann operator acting on $\mathbb{R} \times \Gamma$. In this section we reduce the representation in Proposition 2.5 when $\alpha=\beta=\mathrm{R}$ to the one with the integral on $\mathbb{R} \times \Gamma$ to make the representation more connected with the Neumann operator. This reduced representation is more useful for examinating the singularities of the kernel $S_{\mathrm{RR}}(\cdot, \theta, \omega)$. We continue to use the notations in the previous sections.

The following equality is a basic formula for this reduction:

$$
\left(\partial_{t}+c_{R} \partial_{\omega}\right) w_{0}^{R}(t, x ; \omega)=0 \quad \text { in } \quad \mathbb{R} \times \Omega,
$$

where $\partial_{\omega}=\sum_{i=1}^{2} \omega_{i} \partial_{x_{i}}$ is the tangential derivative on $\partial \mathbb{R}_{+}^{3}$. This is seen from the form of $w_{0}^{R}$ (cf. section 1 or section 5 of [8]). From the above equality, noting that supp $[\psi] \subset B_{r_{0}}^{c}$, we obtain

$$
\begin{aligned}
& \int_{\Omega} \int_{\mathbb{R}} \phi_{2}(s) \partial_{s} \phi_{1}(s) \rho(y) \psi(y) \partial_{s} w_{0}^{R}(s, y ; \omega) \cdot v_{\varphi}^{R}(s, y ; \theta) d s d y \\
& \quad=-\int_{\Omega} \int_{\mathbb{R}} \phi_{2}(s) \partial_{s} \phi_{1}(s) \rho(y) \psi(y) c_{R}\left(\partial_{\omega} w_{0}^{R}\right) \cdot v_{\varphi}^{R} d s d y \\
& \quad=\int_{\Omega} \int_{\mathbb{R}} \rho(y) \phi_{2}(s) \partial_{s} \phi_{1}(s)\left\{\psi w_{0}^{R} \cdot c_{R} \partial_{\omega} v_{\varphi}^{R}+c_{R}\left(\partial_{\omega} \psi\right) w_{0}^{R} \cdot v_{\varphi}^{R}\right\} d s d y .
\end{aligned}
$$


Combining this and Proposition 2.5, we see from (2.8), (2.11) and Lemma 2.6 that $\left\langle\tilde{S}_{\mathrm{RR}}(\cdot, \theta, \omega), \varphi\right\rangle$ is equal to the following $J_{1}^{R}(\varphi)$ modulo negligible terms, i.e., $J_{1}^{R}(\varphi)-$ $\left\langle\tilde{S}_{R R}(\cdot, \theta, \omega), \varphi\right\rangle \simeq 0$ :

$$
J_{1}^{R}(\varphi)=\int_{\Omega} \int_{\mathbb{R}} \rho(x) \phi_{2}(s) \partial_{s} \phi_{1}(s) \psi(x) w_{0}^{R}(s, x ; \omega) \cdot\left(\partial_{s}-c_{R} \partial_{\omega}\right) v_{\varphi}^{R}(s, x ; \theta) d s d x .
$$

The idea to reduce $J_{1}^{R}(\varphi)$ to the form with the integral on $\Gamma$ is as follows. We make an approximate solution $u^{R}(t, x ; \omega)\left(\in C^{2}\left(\overline{\Omega_{x}} ; H^{-\infty}\left(\mathbb{R}_{t}\right)\right)\right)$ such that

$$
\begin{aligned}
& \left\{\begin{array}{lll}
\left(\partial_{t}^{2}-\rho^{-1} \mathcal{A}\right) u^{R}(t, x ; \omega)=\phi_{2}(t) \partial_{t} \phi_{1}(t) \psi(x) w_{0}^{R}(t, x ; \omega) & \bmod & C^{\infty}(\mathbb{R} \times \bar{\Omega}), \\
u^{R}=0 & \bmod & C^{\infty}(\mathbb{R} \times \Gamma), \\
u^{R}(t, x ; \omega)=0 & \text { if }-s_{0}-t_{1}-c_{R}^{-1}<t, &
\end{array}\right. \\
& \operatorname{sing} \operatorname{supp}\left[u^{R}(\cdot, \cdot ; \omega)\right] \subset\left\{(t, x):-s_{0}-t_{1}-2 c_{R}^{-1} \leq t \leq-s_{0}-t_{1}-c_{R}^{-1},\right. \\
& \left.c_{R}\left(-s_{0}-t_{1}-2 c_{R}^{-1}\right) \leq x^{\prime} \cdot \omega \leq c_{R}\left(-s_{0}-t_{1}-c_{R}^{-1}\right), x_{3}=0\right\},
\end{aligned}
$$

and substitute $\left(\partial_{t}^{2}-\rho^{-1} \mathcal{A}\right) u^{R}$ for $\phi_{2}(t) \partial_{t} \phi_{1} \psi w_{0}^{R}$. Then, using integration by parts, we can change the integral on $\Omega$ (in $J_{1}^{R}(\varphi)$ ) for the one on $\Gamma$ modulo negligible terms. Namely we obtain

THEOREM 3.1. We assume that the approximate solution $u^{R}$ in (3.1) and (3.2) is constructed. Take a cutoff function $\psi_{1}(x) \in C^{\infty}\left(\mathbb{R}^{3}\right)$ such that $0 \leq \psi_{1} \leq 1, \psi_{1}(x)=1$ for $|x| \geq r_{0}+2 / 3$ and $\psi_{1}(x)=0$ for $|x| \leq r_{0}+1 / 2$, and $\phi_{0}(t) \in C_{0}^{\infty}(\mathbb{R})$ such that $0 \leq \phi_{0} \leq 1, \phi_{0}(t)=1$ for $t$ with $-s_{0}-t_{1}-5 c_{R}^{-1} / 2 \leq t \leq-s_{0}-t_{1}-c_{R}^{-1} / 2$ and $\phi_{0}(t)=0$ for $t \notin\left[-s_{0}-t_{1}-3 c_{R}^{-1},-s_{0}-t_{1}\right]$. Set

$$
J_{0}(\varphi)=-\int_{\Gamma} \int_{\mathbb{R}} \phi_{0}(s) \psi_{1}(y) \mathcal{N} u^{R}(s, y ; \omega) \cdot\left(\partial_{s}-c_{R} \partial_{\omega}\right) v_{\varphi}^{R}(s, y ; \theta) d s d S_{y} .
$$

Then, $J_{0}(\varphi)-\left\langle\tilde{S}_{\mathrm{RR}}(\cdot, \theta, \omega), \varphi\right\rangle$ is negligible, i.e.,

$$
\left\langle\tilde{S}_{\mathrm{RR}}(\cdot, \theta, \omega), \varphi\right\rangle \simeq J_{0}(\varphi) \text { with respect to } C_{0}^{\infty}(I) \rightarrow \mathbb{C} .
$$

Proof. Since $\left(\partial_{t}^{2}-\rho^{-1} \mathcal{A}\right) u^{R}(t, x ; \omega)=\phi_{2}(t) \partial_{t} \phi_{1}(t) \psi(x) w_{0}^{R}(t, x ; \omega) \bmod C^{\infty}(\mathbb{R} \times$ $\bar{\Omega})$, by Lemma 2.6 we see that

$$
J_{1}^{R}(\varphi) \simeq \int_{\Omega} \int_{\mathbb{R}} \rho\left(\partial_{s}^{2}-\rho^{-1} \mathcal{A}\right) u^{R}(s, y ; \omega) \cdot \phi_{0} \psi_{1}\left(\partial_{s}-c_{R} \partial_{\omega}\right) v_{\varphi}^{R}(s, y ; \theta) d s d y .
$$

Noting that supp $\left[\psi_{1}\right] \subset B_{r_{0}}^{c}$ and applying integration by parts to the right side of the above relation, we can decompose $J_{1}^{R}(\varphi)$ as follows:

$$
\begin{aligned}
& J_{1}^{R}(\varphi) \simeq J_{0}(\varphi)+J_{\Omega,-\infty}^{R}(\varphi)+J_{\Gamma,-\infty}^{R}(\varphi) \\
& J_{\Omega,-\infty}^{R}(\varphi)=\int_{\Omega} \int_{\mathbb{R}} \rho(y) u^{R}(s, y ; \omega) \cdot\left(\partial_{s}^{2}-\rho^{-1} \mathcal{A}\right)\left(\phi_{0} \psi_{1}\left(\partial_{s}-c_{R} \partial_{\omega}\right) v_{\varphi}^{R}\right)(s, y ; \theta) d s d y, \\
& J_{\Gamma,-\infty}^{R}(\varphi)=\int_{\Gamma} \int_{\mathbb{R}} \rho u^{R}(s, y ; \omega) \cdot \phi_{0} \mathcal{N}\left(\psi_{1}\left(\partial_{s}-c_{R} \partial_{\omega}\right) v_{\varphi}^{R}\right)(s, y ; \theta) d s d S_{y} .
\end{aligned}
$$

By the same argument as for the proof of Lemma 2.6, we have

$$
\sup _{y \in \Gamma}\left\|\eta(s) \mathcal{N}\left(\psi_{1}(y)\left(\partial_{s}-c_{R} \partial_{\omega}\right) v_{\varphi}^{R}(s, y ; \theta)\right)\right\|_{H^{m}\left(\mathbb{R}_{s}\right)} \leq C_{\eta, m}\|\varphi\|_{H^{m+l_{0}+2}(\mathbb{R})}
$$


for any integer $m$ and $\eta \in C_{0}^{\infty}\left(I_{t_{1}+3 c_{R}^{-1}}\left(s_{0}\right)\right)$. This implies $J_{\Gamma,-\infty}^{R}(\varphi) \simeq 0$ since $\left.u^{R}\right|_{\mathbb{R} \times \Gamma} \in C^{\infty}(\mathbb{R} \times \partial \Omega)($ cf. (3.1)).

Noting that $\rho(y)=\rho_{0}$ and $\mathcal{A}\left(y, \partial_{y}\right)=\mathcal{A}_{0}\left(\partial_{y}\right)$ in a neighborhood of supp $\left[\psi_{1}(y)\right]$, we can decompose $J_{\Omega,-\infty}^{R}(\varphi)$ as follows way:

$$
\begin{aligned}
J_{\Omega,-\infty}^{R}(\varphi) & =J_{\Omega,-\infty}^{(1)}(\varphi)+J_{\Omega,-\infty}^{(2)}(\varphi)+J_{\Omega,-\infty}^{(3)}(\varphi), \\
J_{\Omega,-\infty}^{(1)}(\varphi) & =\int_{\Omega} \int_{\mathbb{R}} \rho \phi_{0} \psi_{1} u^{R}(s, y ; \omega) \cdot\left(\partial_{s}-c_{R} \partial_{\omega}\right)\left(\left(\partial_{s}^{2}-\rho^{-1} \mathcal{A}\right) v_{\varphi}^{R}\right)(s, y ; \theta) d s d y, \\
J_{\Omega,-\infty}^{(2)}(\varphi) & =\int_{\Omega} \int_{\mathbb{R}} \rho \psi_{1} u^{R}(s, y ; \omega) \cdot\left[\partial_{s}^{2}, \phi_{0}\right]\left(\partial_{s}-c_{R} \partial_{\omega}\right) v_{\varphi}^{R}(s, y ; \theta) d s d y, \\
J_{\Omega,-\infty}^{(3)}(\varphi) & =-\int_{\Omega} \int_{\mathbb{R}} \phi_{0} u^{R}(s, y ; \omega) \cdot\left[\mathcal{A}, \psi_{1}\right]\left(\partial_{s}-c_{R} \partial_{\omega}\right) v_{\varphi}^{R}(s, y ; \theta) d s d y,
\end{aligned}
$$

where $[A, B]$ denotes $A B-B A$. The support of $\left(\partial_{s}^{j} \phi_{0}\right) u^{R}(j \geq 1)$ is contained in $\mathbb{R} \backslash\left[-s_{0}-t_{1}-5 c_{R}^{-1} / 2,-s_{0}-t_{1}-c_{R}^{-1} / 2\right]$. Combining this and (3.2), we obtain $\rho \psi_{1}\left(\partial_{s}^{j} \phi_{0}\right) u^{R}(s, y ; \omega) \in C^{\infty}\left(I_{t_{1}+3 c_{R}^{-1}}\left(s_{0}\right) \times \bar{\Omega}\right)$ for any $j \geq 1$. Therefore, we have $J_{\Omega,-\infty}^{(2)}(\varphi) \simeq 0$ by the same reason that for $J_{\Gamma,-\infty}^{R}(\varphi) \simeq 0$.

Since $-s_{0}-t_{1}<-c_{R}^{-1}\left(r_{0}+4\right),(3.2)$ yields

sing $\operatorname{supp}\left[u^{R}(\cdot, \cdot ; \omega)\right] \subset\left\{(s, y) ;-s_{0}-t_{1}-2 c_{R}^{-1} \leq s \leq-s_{0}-t_{1}-c_{R}^{-1}, r_{0}+5 \leq|y|\right\}$.

Hence, we obtain $\partial_{y}^{\gamma} \psi_{1}(y) u^{R}(s, y ; \omega) \in C^{\infty}\left(I_{t_{1}+3 c_{R}^{-1}}\left(s_{0}\right) \times \bar{\Omega}\right)(|\gamma|>0)$ since supp $\left[\partial_{y_{i}} \psi_{1}(y)\right] \subset\left\{y ; r_{0}+1 / 2<|y|<r_{0}+2 / 3\right\}$. Thus, we have $J_{\Omega,-\infty}^{(3)}(\varphi) \simeq 0$, which is similar to $J_{\Omega,-\infty}^{(2)}(\varphi) \simeq 0$. that

Since $\left(\partial_{t}^{2}-\rho^{-1} \mathcal{A}\right) v_{\varphi}^{R} \simeq \varphi \star\left(\partial_{t} q^{\alpha}\right)$ and $v_{\varphi}^{R}=0$ if $|x| \geq r_{1}$ (cf. (2.9)), it follows

$$
J_{\Omega,-\infty}^{(1)}(\varphi) \simeq \int_{\Omega \cap B_{r_{1}}} \int_{\mathbb{R}} \rho \phi_{0} \psi_{1} u^{R}(s, y ; \omega) \cdot\left(\partial_{s}-c_{R}^{-1} \partial_{\omega}\right) \varphi \star\left(\partial_{s} q^{\alpha}\right)(s, y ; \theta) d s d y .
$$

We have $u^{R} \in C^{2}\left(\bar{\Omega}_{x} ; H^{-\tilde{N}}\left(\mathbb{R}_{t}\right)\right)$ for some $\tilde{N}$, and

$$
\begin{aligned}
\left|\int_{\Omega \cap B_{r_{1}}} \int_{\mathbb{R}} \rho \phi_{0} \psi_{1} u^{R}(s, y ; \omega) \cdot\left(\partial_{s}-c_{R} \partial_{\omega}\right) \varphi \star\left(\partial_{s} q^{R}\right)(s, y ; \theta) d s d x\right| \\
\leq C_{\tilde{N}}\left|B_{r_{1}}\right| \sup _{y \in \overline{\Omega \cap B_{r_{1}}}}\left\|\phi_{0}(\cdot)\left(\partial_{s}-c_{R} \partial_{\omega}\right) \varphi \star\left(\partial_{s} q^{R}\right)(\cdot, y ; \theta)\right\|_{H^{\tilde{N}}(\mathbb{R})} .
\end{aligned}
$$

Noting that supp $\left[\phi_{0}\right] \subset\left[-s_{0}-t_{1}-3 c_{R}^{-1},-c_{R}^{-1}\left(r_{0}+4\right)\right]$, we apply the same argument as for (2.6) to the right side of the above formula. Then, we obtain $\| \phi_{0}(\cdot)\left(\partial_{s}-c_{R} \partial_{\omega}\right) \varphi \star$ $\left(\partial_{s} q^{R}\right)\left\|_{H^{\tilde{N}}(\mathbb{R} \times \Omega)} \leq C_{N}\right\| \varphi \|_{H^{-N}(\mathbb{R})}$ for any integer $N$. This means that $J_{\Omega,-\infty}^{(1)}(\varphi) \simeq 0$. Thus Theorem 3.1 is proved.

Theorem 3.1 means that every piece of information with respect to singularities of $\tilde{S}_{\mathrm{RR}}(s, \theta, \omega)$ at $s=s_{0}$ is contained in the functional $J_{0}(\varphi)$ in Theorem 3.1. Hence, the next step is to construct the approximate solutions $v_{\varphi}^{R}(t, x ; \theta)$ and $u^{R}(t, x ; \omega)$.

4. Approximate solutions with the zero Dirichlet data. In this section and section 5 , we make the approximate solution $v^{R}(t, x ; \theta)$ of the equation (2.7). As 
described in the introduction, we consider the following equations (4.1) and (4.2).

$$
\begin{aligned}
& \left\{\begin{array}{lc}
\left(\partial_{t}^{2}-\rho_{0}^{-1} \mathcal{A}_{0}\right) v^{R, i}(t, x ; \theta)=\varphi \star\left(\partial_{t} q^{R}\right)(t, x ; \theta) & \text { in } I_{t_{1}+3 c_{R}^{-1}}\left(s_{0}\right) \times \mathbb{R}_{+}^{3}, \\
v^{R, i}\left(t, x^{\prime} ; \theta\right)=0 & \text { on } I_{t_{1}+3 c_{R}^{-1}}\left(s_{0}\right) \times \partial \mathbb{R}_{+}^{3},
\end{array}\right. \\
& \left\{\begin{array}{rr}
\left(\partial_{t}^{2}-\rho^{-1} \mathcal{A}\right) v^{R, b}(t, x ; \theta)=0 & \text { in } I_{t_{1}+3 c_{R}^{-1}}\left(s_{0}\right) \times \Omega, \\
\mathcal{N} v^{R, b}(t, x ; \theta)=\varphi \star\left(\partial_{t} m^{R}\right)(t, x ; \theta)-\mathcal{N}\left(\psi_{2}(x) v^{R, i}(t, x ; \theta)\right) & \text { on } I_{t_{1}+3 c_{R}^{-1}}\left(s_{0}\right) \times \Gamma,
\end{array}\right.
\end{aligned}
$$

where $\psi_{2}(x)$ is a cutoff $C^{\infty}$ function such that $\psi_{2}(x)=1$ if $r_{0}+1 / 2 \leq|x| \leq r_{0}+2$ and $\psi_{2}(x)=0$ if $|x| \leq r_{0}+1 / 3$ or $r_{0}+3 \leq|x|$. The coefficients of the operator $\left(\partial_{t}^{2}-\rho^{-1} \mathcal{A}\right)$ coincide with those of $\left(\partial_{t}^{2}-\rho_{0}^{-1} \mathcal{A}_{0}\right)$ in a neighborhood of $\operatorname{supp}\left[q^{R}\right]$ (cf.(2.5)). Furthermore, $\mathrm{WF}\left[q^{R}\right]$ is contained in the elliptic region of the operator $\left(\partial_{t}^{2}-\rho_{0}^{-1} \mathcal{A}_{0}\right)$, and the boundary value problem (4.1) is coercive in a neighborhood of $\mathrm{WF}\left[q^{R}\right]$, i.e., the Lopatinski condition is satisfied there (for the wave front set $\mathrm{WF}[\cdot]$, see [9]). From these facts we see that the solution $v^{R, i}$ of (4.1) satisfies $\left(\partial_{t}^{2}-\right.$ $\left.\rho^{-1} \mathcal{A}\right)\left(\psi_{2} v^{R, i}\right) \simeq \varphi \star\left(\partial_{t} q^{R}\right)$ in $I_{t_{1}+3 c_{R}^{-1}}\left(s_{0}\right) \times \Omega$. This implies that the solution $v^{R}$ of (2.7) is decomposed into the form

$$
v^{R}(t, x ; \theta) \simeq \psi_{2}(x) v^{R, i}(t, x ; \theta)+v^{R, b}(t, x ; \theta) .
$$

Hence, to obtain the approximate solution $v_{\varphi}^{R}(t, x ; \theta)$ satisfying $(2.8)$ and (2.9), we have only to construct approximate solutions $v_{\varphi}^{R, i}$ and $v_{\varphi}^{R, b}$ of $v^{R, i}$ and $v^{R, b}$ respectively.

In this section we construct the approximate solution $v_{\varphi}^{R, i}$ of (4.1). Namely, we make $v_{\varphi}^{R, i}(t, x ; \theta)$ such that for any integer $N \geq 0$

$$
\left\{\begin{array}{cc}
\left(\partial_{t}^{2}-\rho_{0}^{-1} \mathcal{A}_{0}\right) v_{\varphi}^{R, i}(t, x ; \theta) \simeq \varphi \star\left(\partial_{t} q^{\alpha}\right)(t, x ; \theta) & \quad \text { with respect to } C_{0}^{\infty}(I) \rightarrow \mathcal{B}^{N}\left(\overline{I_{t_{1}+3 c_{R}^{-1}}\left(s_{0}\right) \times \mathbb{R}_{+}^{3}}\right) \\
v_{\varphi}^{R, i}\left(t, x^{\prime} ; \theta\right) \simeq 0 & \left(\text { on } \mathbb{R} \times \partial \mathbb{R}_{+}^{3}\right) \\
& \quad \text { with respect to } C_{0}^{\infty}(I) \rightarrow \mathcal{B}^{N}\left(\overline{I_{t_{1}+3 c_{R}^{-1}}\left(s_{0}\right) \times \partial \mathbb{R}_{+}^{3}}\right), \\
v_{\varphi}^{R, i}(t, x ; \theta)=0 & \text { if }|x| \geq r_{1}, \\
v_{\varphi}^{R, i} \simeq 0 \quad \text { with respect to } C_{0}^{\infty}(I) \rightarrow \mathcal{B}^{N}\left(\left[-s_{0}+c_{R}^{-1}\left(r_{0}+3\right)+2 \varepsilon, \infty\right) \times \overline{\mathbb{R}_{+}^{3}}\right) .
\end{array}\right.
$$

Note that the arguments for the construction of $v_{\varphi}^{R, i}(t, x ; \theta)$ do not contain the construction of the scattered Rayleigh wave. However, this is an important step for determining the actual data of the scattered wave. The data are given as the inhomogeneous Neumann data of equation (4.2). The scattered wave is contained in $v^{R, b}(t, x ; \theta)$. The approximation of $v^{R, b}(t, x ; \theta)$ is accomplished in the next section. Note that while constructing $v_{\varphi}^{R, i}$, we also obtain the approximate solution $u^{R}$ in (3.1).

Let $\mathcal{E}_{0}=\left\{(\tau, \xi) \in \mathbb{R} \times \mathbb{R}^{2} ;|\tau|<c_{0}|\xi|\right\}$ for a sufficiently small constant $c_{0}$, and take a $C^{\infty}$ cutoff function $\chi_{0}(\tau, \xi)$ such that $\operatorname{supp}\left[\chi_{0}\right] \subset\left\{(\tau, \xi) \in \mathcal{E}_{0} ;|\tau|^{2}+|\xi|^{2} \geq\right.$ $1 / 2\}$ and that $\chi_{0}(\tau, \xi)=1$ for $(\tau, \xi)$ satisfying $|\tau|^{2}+|\xi|^{2} \geq 1$ and $|\tau| \leq c_{S}\left(1-\varepsilon_{0}\right)|\xi|$ in $\mathcal{E}_{0}$. Here, $\varepsilon_{0}$ is a positive constant that is sufficiently small. We consider the Poisson operator $\mathcal{P}_{\chi_{0}}$ restricted by $\chi_{0}\left(D_{t}, D_{x^{\prime}}\right)$, i.e.,

$$
\mathcal{P}_{\chi_{0}} m(t, x)=(2 \pi)^{-3} \int_{\mathbb{R} \times \mathbb{R}^{2}} e^{i\left(\tau t+\xi \cdot x^{\prime}\right)} \chi_{0}(\tau, \xi) V_{-}^{(0)}\left(x_{3} ; \tau, \xi\right) \hat{m}(\tau, \xi) d \tau d \xi,
$$


where $V_{-}^{(0)}\left(x_{3} ; \tau, \xi\right)$ is the solution of the equation

$$
\left\{\begin{array}{l}
\left(-\tau^{2}-\rho_{0}^{-1} \mathcal{A}_{0}\left(i \xi, \partial_{x_{3}}\right)\right) V_{ \pm}^{(0)}\left(x_{3} ; \tau, \xi\right)=0 \quad \text { in } \mathbb{R} \\
\left.V_{ \pm}^{(0)}(0 ; \tau, \xi)=I \quad \text { (the identity matrix }\right), \\
V_{ \pm}^{(0)}\left(x_{3} ; \tau, \xi\right) \text { is bounded in } \pm x_{3}<0 .
\end{array}\right.
$$

Here, note that $V_{ \pm}^{(0)}$ is of the form

$$
\begin{aligned}
& V_{ \pm}^{(0)}\left(x_{3} ; \tau, \xi\right)=\sum_{\beta=P, S} e^{ \pm \xi_{\beta}^{(0)}(\tau, \xi) x_{3}} P_{\beta}^{ \pm,(0)}(\tau, \xi) \quad\left((\tau, \xi) \in \mathcal{E}_{0}\right), \\
& P_{P}^{ \pm,(0)}(\tau, \xi)=\frac{1}{\xi^{2}-\xi_{P}^{(0)}(\tau, \xi) \xi_{S}^{(0)}(\tau, \xi)} \eta_{P}^{ \pm,(0)}(\tau, \xi) \otimes \eta_{S}^{ \pm,(0)}(\tau, \xi), \\
& P_{S}^{ \pm,(0)}=I-P_{P}^{ \pm,(0)}(\tau, \xi),
\end{aligned}
$$

where $\xi^{2}=\xi_{1}^{2}+\xi_{2}^{2}, \xi_{\beta}^{(0)}(\tau, \xi)=\sqrt{\xi^{2}-\tau^{2} / c_{\beta}^{2}}, \eta_{\beta}^{ \pm,(0)}(\tau, \xi)={ }^{t}\left(\xi_{1}, \xi_{2}, \mp i \xi_{\beta}^{(0)}(\tau, \xi)\right)$ $(\beta=\mathrm{P}, \mathrm{S}), c_{P}=\sqrt{\rho_{0}^{-1}\left(\lambda_{0}+2 \mu_{0}\right)}, c_{S}=\sqrt{\rho_{0}^{-1} \mu_{0}}$ and $a \otimes b=\left(\left.a_{i} b_{j}\right|_{j \rightarrow 1,2,3} ^{i \downarrow 1,2,3}\right)(a=$ $\left.{ }^{t}\left(a_{1}, a_{2}, a_{3}\right), b={ }^{t}\left(b_{1}, b_{2}, b_{3}\right)\right)$.

We employ the Neumann operator $T_{\chi_{0}}$ restricted by $\chi_{0}\left(D_{t}, D_{x^{\prime}}\right)$ :

$$
T_{\chi_{0}} m\left(t, x^{\prime}\right)=\left.\left(\mathcal{N}_{0}\left(\partial_{x}\right) \mathcal{P}_{\chi_{0}} m\right)(t, x)\right|_{\mathbb{R} \times \partial \mathbb{R}_{+}^{3}}, \quad m \in C_{0}^{\infty}\left(\mathbb{R} \times \partial \mathbb{R}_{+}^{3}\right) .
$$

Then, we can see in the same way as [5] or [19] that $T_{\chi_{0}}$ is a Fourier multiplier with the symbol $\rho_{0} \chi_{0}(\tau, \xi) \mathcal{B}^{(0)}(\tau, \xi)$, where $\mathcal{B}^{(0)}(\tau, \xi)=\left.\mathcal{N}_{0}\left(i \xi, \partial_{x_{3}}\right) V_{-}^{(0)}\left(x_{3} ; \tau, \xi\right)\right|_{x_{3}=0}$ and satisfies the following proposition.

Proposition 4.1. (i) Let $\mathcal{R}_{\partial \mathbb{R}_{+}^{3}}=\left\{(\tau, \xi) \in \mathcal{E}_{0} ;|\tau|=c_{R}|\xi|\right\}$. Then, $\mathcal{B}^{(0)}(\tau, \xi)$ is a non-singular matrix if and only if $(\tau, \xi) \notin \mathcal{R}_{\partial \mathbb{R}_{+}^{3}}$.

(ii) $\mathcal{B}^{(0)}(\tau, \xi)$ is Hermit matrix for $(\tau, \xi) \in \mathcal{E}_{0}$.

(iii) There exists a conic neighborhood $\mathcal{E}_{0}^{(0)} \subset \mathcal{E}_{0}$ such that $\mathcal{B}^{(0)}(\tau, \xi)$ has the distinct eigenvalues $\lambda_{j}^{(0)}(\tau, \xi)(j=0,1,2)$ of constant multiplicity possessing the properties

$$
\begin{array}{ll}
\lambda_{0}^{(0)}(\tau, \xi)=\left(c_{R}|\xi|^{2}-\tau^{2}\right) \tilde{\lambda}_{0}^{(0)}(\tau, \xi) & \text { on } \mathcal{E}_{0}^{(0)}, \\
\lambda_{1}^{(0)}(\tau, \xi)>0 & \text { on } \mathcal{E}_{0}^{(0)}, \\
\lambda_{2}^{(0)}(\tau, \xi)=c_{S}^{2} \xi_{S}^{(0)}(\tau, \xi)>0 & \text { on } \mathcal{E}_{0}^{(0)},
\end{array}
$$

where $\tilde{\lambda}_{0}^{(0)}(\tau, \xi)$ is a positive $C^{\infty}$ function homogeneous of order -1 in $(\tau, \xi)$ on $\mathcal{E}_{0}^{(0)}$. (iv) The eigenfunctions of $\lambda_{j}^{(0)}(\tau, \xi)$ are written in the forms

$$
e_{0}^{j,(0)}(\tau, \xi)=\frac{1}{i \tau}\left(\begin{array}{c}
i\left(\frac{\tau^{2}}{\xi^{2}-\xi_{P}^{(0)} \xi_{S}^{(0)}}-2 \mu\right) \xi \\
\lambda_{j}^{(0)}-\frac{\tau^{2} \xi_{P}^{(0)}}{\xi^{2}-\xi_{P}^{(0)} \xi_{S}^{(0)}}
\end{array}\right) \quad(j=0,1), \quad e_{0}^{2,(0)}(\tau, \xi)=\left(\begin{array}{c}
\xi_{2} \\
-\xi_{1} \\
0
\end{array}\right) .
$$

As described in Proposition 4.2 below, the Neumann operator $T_{\chi_{0}}$ is connected closely with the Rayleigh wave $w_{0}^{R}$ (in the free space) of the form $w_{0}^{R}(t, x ; \omega)=$ 
$(2 \pi)^{-1} \int_{\mathbb{R}} e^{i \sigma t} \phi_{0}^{R}(x ;-\sigma, \omega) d \sigma$, where $\phi_{0}^{R}$ is the generalized eigenfunction of $\mathcal{A}_{0}$ corresponding to the Rayleigh wave given in (2.1).

Proposition 4.2. (i) If $f\left(t, x^{\prime}\right) \in \mathcal{S}^{\prime}\left(\mathbb{R} \times \mathbb{R}^{2}\right)$ satisfies $\left(\partial_{t}^{2}-c_{R}^{2} \triangle_{x^{\prime}}\right) f\left(t, x^{\prime}\right)=0$ in $\mathbb{R} \times \mathbb{R}^{2}$, then it follows that $T_{\chi_{0}} e_{0}^{0,(0)}\left(D_{t}, D_{x^{\prime}}\right) f\left(t, x^{\prime}\right)=0$.

(ii) Let $\alpha^{(0)}=c_{S}^{-2} c_{R}\left(1-\xi_{R}^{(1)} \xi_{R}^{(2)}\right) \sqrt{2 \pi \rho_{0}} C_{0}^{R}$. Then, we have

$$
w_{0}^{R}(t, x ; \omega)=\alpha^{(0)} \mathcal{P}_{\chi_{0}} e_{0}^{0,(0)}\left(D_{t}, D_{x^{\prime}}\right)\left(\delta\left(t-c_{R}^{-1} x^{\prime} \cdot \omega\right)\right) \quad \bmod C^{\infty}\left(\mathbb{R} \times \mathbb{R}_{+}^{3}\right) .
$$

Proof. (i) is obtained immediately from $\lambda_{0}^{(0)}(\tau, \xi) \hat{f}(\tau, \xi)=0$. For the proof of (ii), first note that $\xi_{P}^{(0)}=|\tau| \xi_{R}^{(1)} / c_{R}, \xi_{S}^{(0)}=|\tau| \xi_{R}^{(2)} / c_{R}, \eta_{P}^{-,(0)}=-c_{R}^{-1 t}\left({ }^{t} \omega,-i \xi_{R}^{(1)}\right)$ and $\eta_{S}^{-,(0)}=-c_{R}^{-1 t}\left({ }^{t} \omega,-i \xi_{R}^{(2)}\right)$ on $\xi=-c_{R}^{-1} \tau \omega$. Furthermore we note that

$$
\begin{aligned}
& \mathcal{P}_{\chi_{0}} \chi_{0}\left(D_{t}, D_{x^{\prime}}\right) e_{0}^{0,(0)}\left(D_{t}, D_{x^{\prime}}\right)\left(\delta\left(t-c_{R}^{-1} x^{\prime} \cdot \omega\right)\right) \\
& \quad=\left.(2 \pi)^{-1} \int_{\mathbb{R}} e^{i \tau\left(t-c_{R}^{-1} \omega \cdot x^{\prime}\right)}\left(\chi_{0}(\tau, \xi) V_{-}^{(0)}\left(x_{3} ; \tau, \xi\right) e_{0}^{0,(0)}(\tau, \xi)\right)\right|_{\xi=-c_{R}^{-1} \tau \omega} d \tau .
\end{aligned}
$$

$q^{(0)}=\left.\tau^{-2}\left(\xi^{2}-\xi_{P}^{(0)} \xi_{S}^{(0)}\right)\right|_{\xi=-c_{R}^{-1} \tau \omega}$ satisfies $\left(1-2 c_{S}^{2} q^{(0)}\right)^{2}=\xi_{R}^{(1)} \xi_{R}^{(2)}$ since $\left(2-c_{R}^{2} / c_{S}^{2}\right)^{2}-$ $4 \xi_{R}^{(1)} \xi_{R}^{(2)}=0$ (cf. [1]). Therefore we have

$$
\left.\left(V_{-}^{(0)}\left(x_{3} ; \tau, \xi\right) e_{0}^{0,(0)}(\tau, \xi)\right)\right|_{\xi=-c_{R}^{-1} \tau \omega}=\frac{c_{S}^{2}}{c_{R}^{3} q^{(0)} \sqrt{2 \pi \rho_{0}} C_{0}^{R}} e^{-i c_{R}^{-1} \tau \omega \cdot x^{\prime}} \phi_{0}^{R}(x ; \tau, \omega) .
$$

(ii) of Proposition 4.2 is derived from (4.7) and (4.8). Thus, the proof is complete.

To construct the approximate solution $v_{\varphi}^{R, i}(t, x ; \theta)$, we employ the following operator $\mathcal{V}_{\chi_{0}}: q(t, x) \mapsto u(t, x)$ :

$$
\begin{cases}\left(\partial_{t}^{2}-\rho_{0}^{-1} \mathcal{A}_{0}\right) u(t, x)=\chi_{0}\left(D_{t}, D_{x^{\prime}}\right) q\left(t, x^{\prime}, x_{3}\right) & \text { in } \mathbb{R} \times \mathbb{R}_{+}^{3} \\ u\left(t, x^{\prime}\right)=0 & \text { on } \mathbb{R} \times \partial \mathbb{R}_{+}^{3} .\end{cases}
$$

Then, as seen later, we can express $\mathcal{V}_{\chi_{0}}$ concretely by $\mathcal{V}^{(0)}$, which is defined by

$$
\begin{aligned}
\mathcal{V}^{(0)} \hat{q}\left(x_{3} ; \tau, \xi\right)=\int_{0}^{x_{3}} V_{-}^{(0)} & \left(x_{3}-y_{3} ; \tau, \xi\right) \hat{q}\left(y_{3} ; \tau, \xi\right) d y_{3} \\
& +\int_{x_{3}}^{\infty} V_{+}^{(0)}\left(x_{3}-y_{3} ; \tau, \xi\right) \hat{q}\left(y_{3} ; \tau, \xi\right) d y_{3} \\
& -V_{-}^{(0)}\left(x_{3} ; \tau, \xi\right) \int_{0}^{\infty} V_{+}^{(0)}\left(-y_{3} ; \tau, \xi\right) \hat{q}\left(y_{3} ; \tau, \xi\right) d y_{3} .
\end{aligned}
$$

Note that $\mathcal{V}^{(0)} \hat{q}\left(x_{3} ; \tau, \xi\right)$ satisfies

$$
\begin{cases}\left(-\tau^{2}-\rho_{0}^{-1} \mathcal{A}_{0}\left(i \xi, \partial_{x_{3}}\right)\right) \mathcal{V}^{(0)} \hat{q}\left(x_{3} ; \tau, \xi\right)=Q(\tau, \xi) \hat{q}\left(x_{3} ; \tau, \xi\right) & \text { in } x_{3} \geq 0 \\ \mathcal{V}^{(0)} \hat{q}(0 ; \tau, \xi)=0 & \text { on } x_{3}=0 \\ \mathcal{V}^{(0)} \hat{q}\left(x_{3} ; \tau, \xi\right) \text { is bounded in } x_{3} \geq 0 & \end{cases}
$$

where $Q(\tau, \xi)=\rho_{0}^{-1} \mathcal{A}_{0}\left(\nu^{0}\right)\left(\partial_{x_{3}} V_{+}^{(0)}(0 ; \tau, \xi)-\partial_{x_{3}} V_{-}^{(0)}(0 ; \tau, \xi)\right)\left(\nu^{0}={ }^{t}(0,0,-1)\right)$.

We can see from the following Lemma 4.3 that $\mathcal{V}_{\chi_{0}} q$ is of the form

$$
\mathcal{V}_{\chi_{0}} q(t, x)=(2 \pi)^{-3} \int_{\mathbb{R} \times \mathbb{R}^{2}} e^{i\left(\tau t+\xi \cdot x^{\prime}\right)} \chi_{0}(\tau, \xi)\left(\mathcal{V}^{(0)} Q^{-1} \hat{q}\right)\left(x_{3} ; \tau, \xi\right) d \tau d \xi
$$


LEMMA 4.3. (i) $Q(\tau, \xi)$ is symmetric and positive definite for $(\tau, \xi) \in \mathcal{E}_{0}$.

(ii) $\operatorname{For}(\tau, \xi) \in \mathcal{E}_{0}$ it holds that

$$
\left(V_{-}^{(0)}\left(x_{3} ; \tau, \xi\right)\right)^{*}={ }^{t} V_{+}^{(0)}\left(-x_{3} ; \tau, \xi\right)=Q(\tau, \xi) V_{+}^{(0)}\left(-x_{3} ; \tau, \xi\right) Q(\tau, \xi)^{-1} .
$$

Proof. By direct calculation, we see that $Q(\tau, \xi)$ is of the form

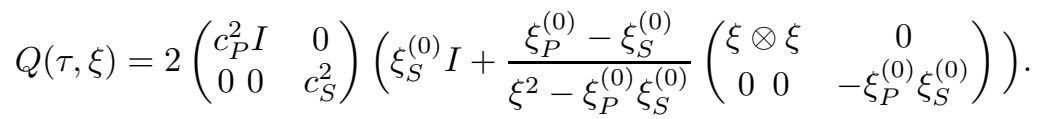

Hence, noting that

$$
\xi_{S}^{(0)}-\left(\xi_{P}^{(0)}-\xi_{S}^{(0)}\right) \xi_{P}^{(0)} \xi_{S}^{(0)} /\left(\xi^{2}-\xi_{P}^{(0)} \xi_{S}^{(0)}\right)=\xi_{S}^{(0)} \tau^{2} / c_{P}^{2}\left(\xi^{2}-\xi_{P}^{(0)} \xi_{S}^{(0)}\right)>0,
$$

we obtain (i).

The first part of the equalities in (ii) is clear from the form (4.6). For the rest, it suffices to show

$$
\int_{0}^{\infty}\left(\left(V_{-}^{(0)}\left(x_{3}\right)\right)^{*} Q \hat{q}\left(x_{3}\right), a\right)_{\mathbb{C}^{3}} d x_{3}=\int_{0}^{\infty}\left(Q V_{+}^{(0)}\left(-x_{3}\right) \hat{q}\left(x_{3}\right), a\right)_{\mathbb{C}^{3}} d x_{3}
$$

for any $a\left(\in \mathbb{C}^{3}\right)$ and $\hat{q}\left(x_{3}\right)$ (omitting the variables $\tau$ and $\xi$ ). Noting that $Q \hat{q}\left(x_{3}\right)=$ $\left(-\tau^{2}-\rho_{0}^{-1} \mathcal{A}_{0}\left(\partial_{x_{3}}\right)\right) \mathcal{V}^{(0)} \hat{q}\left(x_{3}\right)$ and using integration by parts, we see that the left side of (4.12) is equal to

$$
\int_{0}^{\infty}\left(\left(-\tau^{2}-\rho_{0}^{-1} \mathcal{A}_{0}\left(i \xi, \partial_{x_{3}}\right)\right) \mathcal{V}^{(0)} \hat{q}\left(x_{3}\right), V_{-}^{(0)}\left(x_{3}\right) a\right)_{\mathbb{C}^{3}} d x_{3}=-\left(\left.\rho_{0}^{-1} \mathcal{N}_{0}\left(i \xi, \partial_{x_{3}}\right) \hat{q}\right|_{x_{3}=0}, a\right)_{\mathbb{C}^{3}}
$$

Since $P_{\beta}^{ \pm,(0)} P_{\beta^{\prime}}^{ \pm,(0)}=\delta_{\beta, \beta^{\prime}} P_{\beta}^{ \pm,(0)}\left(\beta, \beta^{\prime}=P, S\right)$, we see that $\partial_{x_{3}} V_{ \pm}^{(0)}\left(x_{3}\right)$ is of the form

$$
\partial_{x_{3}} V_{ \pm}^{(0)}\left(x_{3} ; \tau, \xi\right)=\Lambda_{ \pm}(\tau, \xi) V_{ \pm}^{(0)}\left(x_{3} ; \tau, \xi\right), \quad \Lambda_{ \pm}(\tau, \xi)=\left(\partial_{x_{3}} V_{ \pm}^{(0)}\right)(0 ; \tau, \xi)
$$

Combining this and (4.10), and noting the forms of $Q$ and $\mathcal{N}_{0}$ and the fact that $\left.\mathcal{V}^{(0)} \hat{q}\right|_{x_{3}=0}=0$, we have

$$
\left.\mathcal{N}_{0}\left(i \xi, \partial_{x_{3}}\right) \mathcal{V}^{(0)} \hat{q}\right|_{x_{3}=0}=-\rho_{0} Q(\tau, \xi) \int_{0}^{\infty} V_{+}^{(0)}\left(-y_{3} ; \tau, \xi\right) \hat{q}\left(y_{3} ; \tau, \xi\right) d y_{3}
$$

Therefore, the left side of $(4.12)$ is equal to $\int_{0}^{\infty}\left(Q V_{+}^{(0)}\left(-y_{3}\right) \hat{q}\left(y_{3}\right), a\right)_{\mathbb{C}^{3}} d y_{3}$. Hence, (4.12) holds. The proof is complete.

Let $\varphi$ be the function in (4.1), and set $\tilde{\varphi}_{\theta}(t, x)=\varphi\left(c_{R}^{-1} x^{\prime} \cdot \theta-t\right)$. Then, we see from Proposition 4.2 that the following function $v_{\varphi}^{R, i}(t, x ; \theta)$ is an approximate solution of (4.1) (i.e., satisfies (4.4)):

$$
v_{\varphi}^{R, i}=\alpha^{(0)} \mathcal{V}_{\tilde{\chi}_{0}}\left(\left[-\rho_{0}^{-1} \mathcal{A}_{0}\left(\partial_{x}\right), \psi\right] \partial_{t} \mathcal{P}_{\chi_{0}} e_{0}^{0,(0)} \tilde{\varphi}_{\theta}\right),
$$

where the cutoff function $\tilde{\chi}_{0}(\tau, \xi)$ is chosen so that $0 \leq \tilde{\chi}_{0} \leq 1, \operatorname{supp}\left[\tilde{\chi}_{0}\right] \subset \mathcal{E}_{0}$ and $\tilde{\chi}_{0}(\tau, \xi)=1$ in a neighborhood of $\operatorname{supp}\left[\chi_{0}\right]$. Here, note that the operator:

$$
\tilde{\varphi} \mapsto \alpha^{(0)} \mathcal{V}_{\tilde{\chi}_{0}}\left(\left[-\rho_{0}^{-1} \mathcal{A}_{0}\left(\partial_{x}\right), \psi\right] \partial_{t} \mathcal{P}_{\chi_{0}} e_{0}^{0,(0)} \tilde{\varphi}\right)
$$


is a pseudo-differential operator with the parameter $x_{3} \geq 0$.

We can also construct the approximate solution $u^{R}$ in (3.1) and (3.2) in the same way as for $v_{\varphi}^{R, i}$ :

$$
u^{R}(t, x ; \omega)=\psi_{3}(x) \alpha^{(0)} \mathcal{V}_{\tilde{\chi}_{0}}\left(\phi_{2}(t) \partial_{t} \phi_{1}(t) \psi(x) \mathcal{P}_{\chi_{0}} e_{0}^{0,(0)}\left(\delta\left(t-c_{R}^{-1} \omega \cdot x^{\prime}\right)\right)\right) \quad \text { in } \mathbb{R} \times \Omega,
$$

where $\psi_{3}(x)$ is a $C^{\infty}$ cutoff function such that $0 \leq \psi_{3} \leq 1, \psi_{3}(x)=1$ if $r_{0}+2 / 3 \leq|x|$ and $\psi_{3}(x)=0$ if $|x| \leq r_{0}+1 / 2$.

We need to know the properties of $\left.\mathcal{N}\left(\psi_{2} v_{\varphi}^{R, i}\right)\right|_{\mathbb{R} \times \Gamma}$ when constructing the approximate solution $v_{\varphi}^{R, b}(t, x ; \theta)$ of (4.2) in section 5. Since $\operatorname{supp}\left[\psi_{2} v_{\varphi}^{R, i}\right] \subset \mathbb{R} \times B_{r_{0}}^{c}$ it follows that $\left.\left.\mathcal{N}\left(\psi_{2} v_{\varphi}^{R, i}\right)\right|_{\mathbb{R} \times \Gamma} \simeq \mathcal{N}_{0}\left(\psi_{2} v_{\varphi}^{R, i}\right)\right|_{\mathbb{R} \times \partial \mathbb{R}_{+}^{3}}$. Therefore, noting the form (4.15) of $v_{\varphi}^{R, i}$, we have only to examine the operator

$$
F_{0}:\left.\tilde{\varphi}\left(t, x^{\prime}\right) \mapsto \mathcal{N}_{0}\left(\alpha^{(0)} \mathcal{V}_{\tilde{\chi}_{0}}\left[-\rho_{0}^{-1} \mathcal{A}_{0}\left(\partial_{x}\right), \psi\right] \partial_{t} \mathcal{P}_{\chi_{0}} e_{0}^{0,(0)} \tilde{\varphi}\right)\right|_{\mathbb{R} \times \partial \mathbb{R}_{+}^{3}}
$$

$F_{0}$ is a classical pseudo-differential operator of order 1 on $\mathbb{R} \times \partial \mathbb{R}_{+}^{3}$ (for a detailed description of pseudo-differential operators, see Hörmander [2]), and has the following properties:

Lemma 4.4. (i) $F_{0}$ is expressed by a symbol supported in $\operatorname{supp}\left[\nabla_{x^{\prime}}\left(\left.\psi\right|_{\partial \mathbb{R}_{+}^{3}}\right)\right] \times$ $\operatorname{supp}[\chi] \bmod S^{-\infty}$.

(ii) The principal symbol $\sigma_{p}\left(F_{0}\right)$ of $F_{0}$ is of the form

$$
\sigma_{p}\left(F_{0}\right)(t, x, \tau, \xi)=-\alpha^{(0)} \tau \chi_{0}(\tau, \xi) \mathcal{A}_{0}\left(\nu^{0}\right)\left(\nabla_{x^{\prime}} \tilde{\psi}\right)\left(x^{\prime}\right) \cdot \nabla_{\xi} \Lambda_{-}(\tau, \xi) e_{0}^{0,(0)}(\tau, \xi),
$$

where $\Lambda_{ \pm}(\tau, \xi)=\left(\partial_{x_{3}} V_{ \pm}^{(0)}\right)(0 ; \tau, \xi)$ and $\tilde{\psi}\left(x^{\prime}\right)=\psi\left(x^{\prime}, 0\right)$.

(iii) $\sigma_{p}\left(F_{0}\right)$ satisfies

$$
\begin{aligned}
\left(\sigma_{p}\left(F_{0}\right)(t, x, \tau, \xi), e_{0}^{0,(0)}(\tau, \xi)\right)_{\mathbb{C}^{3}=} & \alpha^{(0)} \tau \chi_{0}(\tau, \xi)\left\{\nabla_{x^{\prime}} \tilde{\psi}\left(x^{\prime}\right) \cdot \nabla_{\xi} \lambda_{0}^{(0)}(\tau, \xi)\left|e_{0}^{0,(0)}(\tau, \xi)\right|^{2}\right. \\
& \left.+i\left(\left(\mathcal{N}_{0}\left(\partial_{x}\right) \tilde{\psi}\left(x^{\prime}\right)\right) e_{0}^{0,(0)}(\tau, \xi), e_{0}^{0,(0)}(\tau, \xi)\right)_{\mathbb{C}^{3}}\right\} .
\end{aligned}
$$

Proof. From the definition (4.16) and (4.11), we obtain

$$
F_{0} \tilde{\varphi}=\left.(2 \pi)^{-3} \int_{\mathbb{R} \times \mathbb{R}^{2}} e^{i\left(\tau t+\xi \cdot x^{\prime}\right)} \chi_{0}(\tau, \xi) \mathcal{N}_{0}\left(i \xi, \partial_{x_{3}}\right) \mathcal{V}^{(0)} Q^{-1} \hat{h} d \tau d \xi\right|_{x_{3}=0}
$$

with $h=\alpha^{(0)}\left[-\rho_{0}^{-1} \mathcal{A}_{0}\left(\partial_{x}\right), \psi\right] \partial_{t} \mathcal{P}_{\chi_{0}} e_{0}^{0,(0)} \tilde{\varphi}$. Using (4.14) and (ii) of Lemma 4.3, we have

$$
\begin{aligned}
F_{0} \tilde{\varphi}=(2 \pi)^{-3} & \int_{\mathbb{R} \times \mathbb{R}^{2}} e^{i\left(\tau t+\xi \cdot x^{\prime}\right)} \alpha^{(0)} \rho_{0} \tilde{\chi}_{0}(\tau, \xi) \\
& \int_{0}^{\infty}\left(V_{-}^{(0)}\left(y_{3} ; \tau, \xi\right)\right)^{*} \mathcal{F}^{\prime}\left[\left[\rho_{0}^{-1} \mathcal{A}_{0}, \psi\right] \partial_{t} \mathcal{P}_{\chi_{0}} e_{0}^{0,(0)} \tilde{\varphi}\right]\left(\tau, \xi, y_{3}\right) d y_{3} d \tau d \xi,
\end{aligned}
$$

where $\mathcal{F}^{\prime}$ is the Fourier transformation in $\left(t, x^{\prime}\right)$. From this and $(4.5)$, we see that $F_{0}$ is a pseudo-differential operator represented by the following double symbol in the variables $\in \mathbb{R}_{\left(t, x^{\prime}\right)}^{3} \times \mathbb{R}_{(\tau, \xi)}^{3} \times \mathbb{R}_{\left(s, y^{\prime}\right)}^{3} \times \mathbb{R}_{(\tilde{\tau}, \tilde{\xi})}^{3}$ :

$$
\begin{aligned}
p\left(t, x^{\prime}, \tau, \xi, s, y^{\prime}, \tilde{\tau}, \tilde{\xi}\right) & =i \alpha^{(0)} \tau \rho_{0} \tilde{\chi}_{0}(\tau, \xi) \int_{0}^{\infty}\left(V_{-}^{(0)}\left(y_{3} ; \tau, \xi\right)\right)^{*} \\
& {\left[\rho_{0}^{-1} \mathcal{A}_{0}, \psi\right]\left(y^{\prime}, y_{3} ; i \tilde{\xi}, \partial_{y_{3}}\right) V_{-}^{(0)}\left(y_{3} ; \tilde{\tau}, \tilde{\xi}\right) d y_{3} \chi_{0}(\tilde{\tau}, \tilde{\xi}) e_{0}^{0,(0)}(\tilde{\tau}, \tilde{\xi}), }
\end{aligned}
$$


which yields (i).

Since $\left|\partial_{(\tau, \xi)}^{\alpha} \partial_{y_{3}}^{l} V_{-}^{(0)}\left(y_{3} ; \tau, \xi\right)\right| \leq C_{\alpha, l} e^{-c_{0}(|\tau|+|\xi|) y_{3}}(1+|\tau|+|\xi|)^{l-|\alpha|}\left(y_{3} \geq 0,(\tau, \xi) \in\right.$ $\left.\mathbb{R} \times \mathbb{R}^{2},|\tau|+|\xi| \geq 1\right)$ and $\int_{0}^{\infty} y_{3}^{l} V_{-}^{(0)}\left(y_{3} ; \tau, \xi\right) d y_{3}=O\left((|\tau|+|\xi|)^{-1-l}\right)(\operatorname{as}|\tau|+|\xi| \rightarrow \infty)$, it is seen from the form of $p\left(t, x^{\prime}, \tau, \xi, s, y^{\prime}, \tilde{\tau}, \tilde{\xi}\right)$ that the principal symbol of $F_{0}$ is of the form

$$
\begin{aligned}
\sigma_{p}\left(F_{0}\right)\left(t, x^{\prime}, \tau, \xi\right) & =\alpha^{(0)} \tau \rho_{0} \chi_{0}(\tau, \xi) \int_{0}^{\infty}\left(V_{-}^{(0)}\left(y_{3} ; \tau, \xi\right)\right)^{*} \\
& \sigma_{p}\left(\left[\rho_{0}^{-1} \mathcal{A}_{0}, \tilde{\psi}\right]\right)\left(x^{\prime} ; i \xi, \partial_{y_{3}}\right) V_{-}^{(0)}\left(y_{3} ; \tau, \xi\right) e_{0}^{0,(0)}(\tau, \xi) d y_{3} .
\end{aligned}
$$

Let $\tilde{\xi}(s)=\xi+s \nabla_{x^{\prime}} \tilde{\psi}\left(x^{\prime}\right)$. Then we have

$$
i \sigma_{p}\left(\left[\mathcal{A}_{0}\left(\partial_{x}\right), \tilde{\psi}\right]\right)\left(x^{\prime}, \xi, \xi_{3}\right)=\left.\partial_{s}\left(\mathcal{A}_{0}\left(i \tilde{\xi}(s), i \xi_{3}\right)\right)\right|_{s=0} .
$$

Since $v_{a}\left(y_{3} ; s\right)=V_{-}^{(0)}\left(y_{3} ; \tau, \tilde{\xi}(s)\right) a\left(a \in \mathbb{C}^{3}\right)$ satisfies

$$
\left(-\tau^{2}-\rho_{0}^{-1} \mathcal{A}_{0}\left(i \tilde{\xi}(s), \partial_{y_{3}}\right)\right) v_{a}\left(y_{3}, s\right)=0
$$

we obtain

$$
\left.\frac{\partial}{\partial s} \int_{0}^{\infty}\left(\left(-\tau^{2}-\rho_{0}^{-1} \mathcal{A}_{0}\left(i \tilde{\xi}(s), \partial_{y_{3}}\right)\right) v_{a}\left(y_{3}, s\right), v_{b}\left(y_{3}, s\right)\right)_{\mathbb{C}^{3}} d y_{3}\right|_{s=0}=0
$$

for any $a, b \in \mathbb{C}^{3}$. Combining this, (4.18) and $v_{a}(0, s)=a$ and using integration by parts, we have

$$
\begin{aligned}
\rho_{0}^{-1}\left(\sigma_{p}\left(\left[\mathcal{A}_{0}, \tilde{\psi}\right]\right)\left(x^{\prime}, i \xi, \partial_{x_{3}}\right)\right. & \left.v_{a}(\cdot, 0), v_{b}(\cdot, 0)\right)_{L^{2}([0, \infty))} \\
& =-\rho_{0}^{-1}\left(\mathcal{A}_{0}\left(\nu^{0}\right) \partial_{x_{3}} \partial_{s} v_{a}(0,0), b\right)_{\mathbb{C}^{3}} .
\end{aligned}
$$

(4.13) yields $\left.\partial_{s} \partial_{x_{3}} V_{-}^{(0)}(0 ; \tau, \tilde{\xi}(s))\right|_{s=0}=\left.\partial_{s}\left(\Lambda_{-}(\tau, \tilde{\xi}(s))\right)\right|_{s=0}=\left(\nabla_{x^{\prime}} \tilde{\psi}\left(x^{\prime}\right) \cdot \nabla_{\xi}\right) \Lambda_{-}(\tau, \xi)$. Therefore, (ii) of Lemma 4.4 follows from (4.19) and (4.17).

Since $\mathcal{B}^{(0)}(\tau, \xi) e_{0}^{0,(0)}(\tau, \xi)=\lambda_{0}^{(0)}(\tau, \xi) e_{0}^{0,(0)}(\tau, \xi) \quad$ and $\quad \mathcal{B}^{(0)}(\tau, \xi)=$ $-\mathcal{A}_{0}\left(\nu^{0}\right) \Lambda_{-}(\tau, \xi)+\mathcal{N}_{0}(i \xi, 0)$, we have $\mathcal{A}_{0}\left(\nu^{0}\right) \Lambda_{-} e_{0}^{0,(0)}=-\lambda_{0}^{(0)} e_{0}^{0,(0)}+\mathcal{N}_{0}(i \xi, 0) e_{0}^{0,(0)}$ and $\left(\mathcal{A}_{0}\left(\nu^{0}\right) \Lambda_{-}\right)^{*} e_{0}^{0,(0)}=-\lambda_{0}^{(0)} e_{0}^{0,(0)}+\left(\mathcal{N}_{0}(i \xi, 0)\right)^{*} e_{0}^{0,(0)}$. Here, note that $\mathcal{B}^{(0)}(\tau, \xi)$ is a Hermite matrix. (iii) of Lemma 4.4 is derived from these relations and $\left(\nabla_{x^{\prime}} \tilde{\psi} \cdot \nabla_{\xi}\right) \mathcal{N}_{0}(i \xi, 0)=-i\left(\mathcal{N}_{0}\left(\partial_{x}\right) \tilde{\psi}\right)\left(x^{\prime}\right)$. The proof is complete.

By the above arguments, we can find the concrete form of the boundary data in (4.2) as follows. Choose the function $\psi(x)$ so that $\psi$ depends only on $|x|$. Then, we have $\partial_{x_{3}} \psi\left(x^{\prime}, 0\right)=0$; by (ii) of Proposition 4.2

$$
\varphi \star\left(\partial_{t} m^{R}\right)\left(t, x^{\prime} ; \theta\right) \simeq\left(\mathcal{N}_{0} \tilde{\psi}\right) \partial_{t} \chi_{0}\left(D_{t}, D_{x^{\prime}}\right) e_{0}^{0,(0)}\left(D_{t}, D_{x^{\prime}}\right)\left(\alpha^{(0)} \tilde{\varphi}_{\theta}\right)\left(t, x^{\prime}\right) .
$$

Since $\left.\mathcal{N}\left(\psi_{2} v_{\varphi}^{R, i}\right)\right|_{\mathbb{R} \times \Gamma} \simeq \psi_{2} F_{0} \tilde{\varphi}$, we can see that the boundary data of (4.2) are approximated by the operator

$$
F_{1}=\alpha^{(0)}\left(\mathcal{N}_{0} \tilde{\psi}\right) \partial_{t} \chi_{0}\left(D_{t}, D_{x^{\prime}}\right) e_{0}^{0,(0)}\left(D_{t}, D_{x^{\prime}}\right)-\psi_{2} F_{0} .
$$

Namely, using (4.20), (4.16) and Lemma 4.4, we can verify the following lemma:

Proposition 4.5. (i) $F_{1}$ is a classical pseudo-differential operator of order 1 on $\mathbb{R} \times \partial \mathbb{R}_{+}^{3}$ (i.e. $F_{1} \in \Psi_{\text {phg }}^{1}\left(\mathbb{R} \times \partial \mathbb{R}_{+}^{3} ; \mathbb{C}, \mathbb{C}^{3}\right)$, which is defined in [2]), and is expressed 
by a symbol supported in $\operatorname{supp}\left[\nabla_{x^{\prime}}\left(\left.\psi\right|_{\partial \mathbb{R}_{+}^{3}}\right)\right] \times \operatorname{supp}[\chi] \bmod S^{-\infty}$. Furthermore, the principal symbol $\sigma_{p}\left(F_{1}\right)$ satisfies

$$
\left(\sigma_{p}\left(F_{1}\right)(t, x, \tau, \xi), e_{0}^{0,(0)}(\tau, \xi)\right)_{\mathbb{C}^{3}}=-\alpha^{(0)} \tau \chi_{0}(\tau, \xi) \nabla_{x^{\prime}} \tilde{\psi}\left(x^{\prime}\right) \cdot \nabla_{\xi} \lambda_{0}^{(0)}(\tau, \xi)\left|e_{0}^{0,(0)}(\tau, \xi)\right|^{2}
$$

(ii) $F_{1} \tilde{\varphi}_{\theta}$ is approximate to the boundary data in (4.2), i.e., for any integer $N \geq 0$

$\varphi \star\left(\partial_{t} m^{R}\right)-\left.\mathcal{N}\left(\psi_{2} v_{\varphi}^{R, i}\right)\right|_{\mathbb{R} \times \Gamma} \simeq \psi_{1} F_{1} \tilde{\varphi}_{\theta}$ with respect to $C_{0}^{\infty}(I) \rightarrow \mathcal{B}^{N}\left(\overline{I_{t_{1}+3 c_{R}^{-1}}\left(s_{0}\right) \times \Gamma}\right)$.

By the same method as for the proof of Lemma 4.4, we can express $\left.\mathcal{N} u^{R}\right|_{\mathbb{R} \times \Gamma}$ with a pseudo-differential operator. Namely, there exists a pseudo-differential operator $G_{0}$ of order -1 such that $\left.\mathcal{N} u^{R}\right|_{\mathbb{R} \times \Gamma}=G_{0}\left(\delta\left(t-c_{R}^{-1} \omega \cdot x^{\prime}\right)\right) \bmod C^{\infty}(\mathbb{R} \times \Gamma)$, and the principal symbol of $G_{0}$ is of the form

$$
\begin{aligned}
\sigma_{p}\left(G_{0}\right)\left(t, x^{\prime}, \tau, \xi\right)=-\alpha^{(0)} \rho_{0} \chi_{0}(\tau, \xi) \phi_{2}(t) \partial_{t} \phi_{1}(t) \tilde{\psi}\left(x^{\prime}\right) \\
\int_{0}^{\infty}\left(V_{-}^{(0)}\left(y_{3} ; \tau, \xi\right)\right)^{*} V_{-}^{(0)}\left(y_{3} ; \tau, \xi\right) e_{0}^{0,(0)}(\tau, \xi) d y_{3} .
\end{aligned}
$$

The lower symbols of the asymptotic expansion of $G_{0}$ contain derivatives of $\phi_{2} \partial_{t} \phi_{1}$ and $\tilde{\psi}$, and it holds for any $l \geq 1,|\alpha| \geq 1$ that $\left.\left(\partial_{t}^{l}\left(\phi_{2}(t) \partial_{t} \phi_{1}(t)\right) \partial_{x^{\prime}}^{\alpha} \tilde{\psi}\left(x^{\prime}\right)\right)\right|_{t=c_{R}^{-1} \omega \cdot x^{\prime}}=0$ $\left(x^{\prime} \in \partial \mathbb{R}_{+}^{3}\right)$. This implies that $\left.\mathcal{N} u^{R}\right|_{\mathbb{R} \times \Gamma}=\sigma_{p}\left(G_{0}\right)\left(t, x^{\prime}, D_{t}, D_{x^{\prime}}\right)\left(\delta\left(t-c_{R}^{-1} \omega \cdot x^{\prime}\right)\right) \bmod$ $C^{\infty}(\mathbb{R} \times \Gamma)$. Noting this fact and the equality $\operatorname{supp}\left[\phi_{2} \partial_{t} \phi_{1} \tilde{\psi}\right] \cap \operatorname{supp}\left[1-\phi_{0} \psi_{1}\right]=\emptyset$, we can rewrite the expression of $\left.\mathcal{N} u^{R}\right|_{\mathbb{R} \times \Gamma}$ using the pseudo-differential operator $G_{1}$ of order -1 that satisfies

$$
\begin{gathered}
\left.\mathcal{N} u^{R}\right|_{\mathbb{R} \times \Gamma}=G_{1}\left(\phi_{2}(t) \partial_{t} \phi_{1}(t) \tilde{\psi}\left(x^{\prime}\right) \delta\left(t-c_{R}^{-1} \omega \cdot x^{\prime}\right)\right) \quad \bmod \quad C^{\infty}(\mathbb{R} \times \Gamma), \\
\begin{array}{r}
\sigma_{p}\left(G_{1}\right)\left(t, \tau, x^{\prime}, \xi\right)=-\alpha^{(0)} \rho_{0} \chi_{0}(\tau, \xi) \phi_{0}(t) \psi_{1}\left(x^{\prime}\right) \\
\int_{0}^{\infty}\left(V_{-}^{(0)}\left(y_{3} ; \tau, \xi\right)\right)^{*} V_{-}^{(0)}\left(y_{3} ; \tau, \xi\right) e_{0}^{0,(0)}(\tau, \xi) d y_{3}, \\
\operatorname{supp}\left[\sigma\left(G_{1}\right)\left(t, \tau, x^{\prime}, \xi\right)\right] \subset \operatorname{supp}[\phi] \times \operatorname{supp}[\tilde{\psi}] \times \mathbb{R}_{(\tau, \xi)}^{3} \\
\cap\left\{(t, \tau, \zeta) \in T^{*}(\mathbb{R} \times \Gamma) ; r_{0}+1 \leq\left|\pi_{\Gamma}(\zeta)\right|,\right. \\
\left.-\left(t_{1}+2 c_{R}^{-1}\right) \leq t+s_{0} \leq-\left(t_{1}+c_{R}^{-1}\right)\right\},
\end{array}
\end{gathered}
$$

where $\sigma\left(G_{1}\right)\left(t, \tau, x^{\prime}, \xi\right)$ denotes the full symbol of $G_{1}$, and $\pi_{\Gamma}: T^{*}(\Gamma) \rightarrow \Gamma$ is the projection to $\Gamma$.

5. Approximate solutions with inhomogeneous Neumann data. In this section we construct the approximate solution $v_{\varphi}^{R, b}$ of (4.3) using the Neumann operator $T^{ \pm} . T^{ \pm}$are operators of the forms $T^{ \pm}=\mathcal{N} \mathcal{P}^{ \pm}$. Here, $\mathcal{P}^{ \pm}$denotes the Poisson operators: $m\left(t, x^{\prime}\right) \mapsto w^{ \pm}(t, x)$, where $w^{ \pm}(t, x)$ are the solutions of the equations

$$
\begin{cases}\left(\rho \partial_{t}^{2}-\mathcal{A}\right) w^{ \pm}(t, x)=0 & \text { in } \mathbb{R} \times \Omega \\ w^{ \pm}\left(t, x^{\prime}\right)=m\left(t, x^{\prime}\right) & \text { on } \mathbb{R} \times \Gamma \\ w^{ \pm}(t, x)=0 \text { if } \mp t(>1) \text { is sufficiently large. } & \end{cases}
$$

Hereafter, when expressing points on the boundary $\Gamma$, we use the notations $x^{\prime}, y^{\prime}$, etc. Therefore, the points on $\Gamma \cap B_{r_{0}}^{c}$ are written as $x^{\prime}={ }^{t}\left(x_{1}, x_{2}, 0\right)$. In the previous 
sections $x^{\prime}={ }^{t}\left(x_{1}, x_{2}\right)$ represents the first two components of $x={ }^{t}\left(x_{1}, x_{2}, x_{3}\right)$. Although the notation $x^{\prime}$ contains these different meanings, we use it since it is simple and does not cause confusion.

For $m \in C^{\infty}(\mathbb{R} \times \Gamma)$ vanishing for large $\pm t>>1$, we put $u(t, x)=$ $\mathcal{P}^{ \pm}\left(T^{ \pm}\right)^{-1} m(t, x)$. Then, this function $u(t, x)$ becomes the solution for the elastic wave equation with the inhomogeneous Neumann condition $\mathcal{N}\left(x, \partial_{x}\right) u=m$ on $\mathbb{R} \times \Gamma$. This implies that the construction of $v_{\varphi}^{R, b}$ can be reduced to that of the inverse of $T^{ \pm}$. In the elliptic region of $\rho \partial_{t}^{2}-\mathcal{A}, T^{ \pm}$are given by a pseudo-differential operator on $\mathbb{R} \times \Gamma$ modulo smoothing operators. Taylor [19] investigated propagation of singularities of the Rayleigh wave by using this expression of the Neumann operator as pseudo-differential operators. Our construction of $v_{\varphi}^{R, b}$ essentially follows Taylor's outline.

The first step is to examine the principal symbol of $T^{-}$that is restricted in the elliptic region of $\left(\rho \partial_{t}^{2}-\mathcal{A}\right)$. We denote points in $T^{*}(\mathbb{R})$ and $T^{*}(\Gamma)$ by $(t, \tau)$ and $\zeta=\left(x^{\prime}, \zeta_{x^{\prime}}\right)$ respectively, and by $\|\zeta\|_{\Gamma}$ the metric on $T^{*}(\Gamma)$ induced by the standard Riemann metric of $\Gamma$. Let $\mathcal{E}$ be the elliptic region of $\rho \partial_{t}^{2}-\mathcal{A}$, i.e., $\mathcal{E}=\{(t, \tau, \zeta) \in$ $\left.T^{*}(\mathbb{R} \times \Gamma) ;|\tau|<\tilde{c}_{S}\left(x^{\prime}\right)\|\zeta\|_{\Gamma}\right\}$ for $\tilde{c}_{S}\left(x^{\prime}\right)=\sqrt{\mu\left(x^{\prime}\right) / \rho\left(x^{\prime}\right)}$.

Hereafter, when we take a conic neighborhood $V$ in $T^{*}(\mathbb{R} \times \Gamma)$, we always choose $V$ so that $\check{I}_{\mathbb{R} \times \Gamma}(V)=V$ for the operator $\check{I}_{\mathbb{R} \times \Gamma}:(t, \tau, \zeta)=\left(t, \tau, x^{\prime}, \zeta_{x^{\prime}}\right) \mapsto\left(t,-\tau, x^{\prime},-\zeta_{x^{\prime}}\right)$. Let $\tilde{c}_{R}\left(x^{\prime}\right)$ be the propagation speed of the Rayleigh wave at $x^{\prime} \in \Gamma$, and set $\mathcal{R}_{\Gamma}=$ $\left\{(t, \tau, \zeta) \in T^{*}(\mathbb{R} \times \Gamma) ; \tilde{c}_{R}\left(x^{\prime}\right)\|\zeta\|_{\Gamma}=|\tau|\right\}$. Note that $\tilde{c}_{R}\left(x^{\prime}\right)$ is given by $\tilde{c}_{R}\left(x^{\prime}\right)=$ $\tilde{c}_{S}\left(x^{\prime}\right) s_{0}\left(x^{\prime}\right)$, where $s=s_{0}\left(x^{\prime}\right)$ is the unique root of the following equation in $s \in(0,1)$ :

$$
s^{3}-8 s^{2}+8\left(3-2 \frac{\mu\left(x^{\prime}\right)}{\lambda\left(x^{\prime}\right)+2 \mu\left(x^{\prime}\right)}\right) s+16\left(\frac{\mu\left(x^{\prime}\right)}{\lambda\left(x^{\prime}\right)+2 \mu\left(x^{\prime}\right)}-1\right)=0 .
$$

We take conic neighborhoods $V_{0}, V_{1}$ and $\tilde{V}_{1}$ such that $\mathcal{R}_{\Gamma} \subset V_{1} \subset \subset \tilde{V}_{1} \subset \subset V_{0} \subset \subset \mathcal{E}$, where $A \subset \subset B$ means that $\bar{A} \subset B$. Let $W_{0}=\left\{(t, \tau, \zeta) \in V_{0} ;\left|t+s_{0}\right|<t_{1}+8 c_{R}^{-1}\right\}$, $W_{1}=\left\{(t, \tau, \zeta) \in V_{1} ;\left|t+s_{0}\right|<t_{1}+6 c_{R}^{-1}\right\}$ and $\tilde{W}_{1}=\left\{(t, \tau, \zeta) \in \tilde{V}_{1} ;\left|t+s_{0}\right|<t_{1}+\right.$ $\left.7 c_{R}^{-1}\right\}$, and take a cutoff function $\chi_{1}(t, \tau, \zeta) \in C^{\infty}\left(T^{*}(\mathbb{R} \times \Gamma)\right)$ such that $0 \leq \chi_{1} \leq 1$, $\operatorname{supp}\left[\chi_{1}\right] \subset W_{0}, \chi_{1}(t, \tau, \zeta)=1$ on $\tilde{W}_{1}$ and $\chi_{1}(t, \tau, \zeta)=0$ near the zero section of $T^{*}(\mathbb{R} \times \Gamma)$.

Hereafter, we use the notations in Hörmander [2] for pseudo-differential operators on manifolds $\mathrm{M}$ (e.g., the classes of the pseudo-differential operators $\Psi_{p h g}^{m}(M)$, $\Psi^{ \pm \infty}(M)$, etc.). As is checked by the method presented in Chapter 6 of Kumano-go [9], the operator

$$
B_{\chi_{1}}=T^{-} O p\left(\chi_{1}\right)
$$

is known to possess the following properties (cf., e.g., [5] or [19]).

Proposition 5.1. (i) Let $V_{0}$ be sufficiently small. Then, we have $B_{\chi_{1}} \in \Psi_{p h g}^{1}(\mathbb{R} \times$ $\left.\Gamma ; \mathbb{C}^{3}, \mathbb{C}^{3}\right)$. Furthermore, there exists a (matrix-valued) function $B_{1}(\tau, \zeta)\left(\in C^{\infty}\left(\overline{V_{0}}\right)\right)$ homogeneous of order one (depending only on $\Gamma, \rho, \lambda$ and $\mu$ ) such that

$$
\sigma_{p}\left(B_{\chi_{1}}\right)(t, \tau, \zeta)=\chi_{1}(t, \tau, \zeta) B_{1}(\tau, \zeta) \quad \text { on } \quad T^{*}(\mathbb{R} \times \Gamma)
$$

(ii) The above $B_{1}(\tau, \zeta)$ is a Hermite matrix, and has the distinct eigenvalues $\lambda^{(e), j}(\tau, \zeta)$ $(j=0,1,2)$ of constant multiplicity. Moreover, $\lambda^{(e), j}(\tau, \zeta)>0$ holds on $\overline{V_{0}}$ for $j=$ 1,2 , and $\lambda^{(e), 0}(\tau, \zeta)$ is of the form

$$
\lambda^{(e), 0}(\tau, \zeta)=\left(\tilde{c}_{R}\left(x^{\prime}\right)\|\zeta\|_{\Gamma}-|\tau|\right) \tilde{\lambda}^{(e), 0}(\tau, \zeta), \quad(\tau, \zeta)=\left(\tau, x^{\prime}, \zeta_{x^{\prime}}\right) \in V_{0}^{\prime},
$$


where $V_{0}^{\prime}=\left\{(\tau, \zeta) \in \mathbb{R} \times T^{*}(\Gamma) ;(t, \tau, \zeta) \in V_{0}\right.$ for some $\left.t \in \mathbb{R}\right\}$ and $\tilde{\lambda}^{(e), 0}(\tau, \zeta) \in$ $C^{\infty}\left(\overline{V_{0}^{\prime}}\right)$ is homogeneous of order 0 and satisfies $\tilde{\lambda}^{(e), 0}(\tau, \zeta)>0$ on $\overline{V_{0}^{\prime}}$.

(iii) For any local coordinate $\kappa: \mathbb{R} \times \tilde{U} \ni(t, \sigma) \mapsto(t, s(\sigma)) \in \mathbb{R} \times \Gamma$, the local symbol $\left.\sigma\left(B_{\chi_{1}}\right)\right|_{\mathbb{R} \times \tilde{U}}$ of $B_{\chi_{1}}$ satisfies

$$
\left.\sigma\left(B_{\chi_{1}}\right)\right|_{\mathbb{R} \times \tilde{U}}(t,-\tau, \sigma,-\xi)=\overline{\left.\sigma\left(B_{\chi_{1}}\right)\right|_{\mathbb{R} \times \tilde{U}}(t, \tau, \sigma, \xi)} .
$$

$O p\left(\chi_{1}\right)$ restricts the boundary data to the elliptic region of $\left(\rho \partial_{t}^{2}-\mathcal{A}\right)$ and to the finite interval in $\mathbb{R}_{t}$. This implies that $\left(B_{\chi_{1}}=\right) T^{-} O p\left(\chi_{1}\right)=T^{+} O p\left(\chi_{1}\right) \bmod$ $\Psi_{p h g}^{-\infty}(\mathbb{R} \times \Gamma)$. From this fact, we see that

$$
\left(O p\left(\chi_{1}\right)\right)^{*} B_{\chi_{1}}-\left(B_{\chi_{1}}\right)^{*} O p\left(\chi_{1}\right) \in \Psi_{p h g}^{-\infty}(\mathbb{R} \times \Gamma) .
$$

In fact, using integration by parts, we can check that for any $f, g \in C_{0}^{\infty}(\mathbb{R} \times \Gamma)$

$$
\left(T^{-} O p\left(\chi_{1}\right) f, O p\left(\chi_{1}\right) g\right)_{L^{2}(\mathbb{R} \times \Gamma)}=\left(O p\left(\chi_{1}\right) f, T^{+} O p\left(\chi_{1}\right) g\right)_{L^{2}(\mathbb{R} \times \Gamma)} .
$$

Therefore, we obtain (5.2) since $B_{\chi_{1}}=T^{-} O p\left(\chi_{1}\right)=T^{+} O p\left(\chi_{1}\right) \bmod \Psi_{p h g}^{-\infty}(\mathbb{R} \times \Gamma)$.

From Proposition 5.1, two eigenvalues $\lambda^{(e), j}(\tau, \zeta)(\mathrm{j}=1,2)$ are positive, which are elliptic parts. On the other hand, $\lambda^{(e), 0}(\tau, \zeta)$ is a symbol of the real principal type. Thus, this component is hyperbolic. Using the properties of the principal symbol of $B_{\chi_{1}}$, we decompose $B_{\chi_{1}}$ into elliptic and hyperbolic parts with a symbol of the real principal type.

Definition 5.2. For $P, Q \in \Psi^{\infty}(\mathbb{R} \times \Gamma)$, and conic neighborhoods $V_{0}$ and $V_{1}$ with $V_{1} \subset \subset V_{0} \subset T^{*}\left(\mathbb{R} \times \Gamma\right.$ ), we say that $P=Q \bmod \Psi_{V_{1}, V_{0}}^{-\infty}(\mathbb{R} \times \Gamma)$ if (ess.supp $P \cup$ ess.supp $Q) \subset V_{0}$ and $P X-Q X, X P-X Q \in \Psi^{-\infty}(\mathbb{R} \times \Gamma)$ for any $X \in \Psi_{\text {phg }}^{0}(\mathbb{R} \times \Gamma)$ with ess.supp $X \subset V_{1}$.

Let $e_{0}^{(e)}(\tau, \zeta)$ be the eigenvector of $\lambda^{(e), 0}(\tau, \zeta)$, and set

$$
\begin{aligned}
& P_{0}(\tau, \zeta)=I-\left|e_{0}^{(e)}(\tau, \zeta)\right|^{-2} e_{0}^{(e)}(\tau, \zeta) \otimes\left(e_{0}^{(e)}(\tau, \zeta)\right)^{*}, \\
& Q_{1}(\tau, \zeta)=B_{1}(\tau, \zeta)+|\tau| \tilde{\lambda}^{(e), 0}(\tau, \zeta)\left(I-P_{0}(\tau, \zeta)\right) .
\end{aligned}
$$

Note that $P_{0}(\tau, \zeta)$ is the projection to the orthogonal complement of the eigenspace for $\lambda^{(e), 0}(\tau, \zeta)$. From Proposition 5.1, we see that $Q_{1}(\tau, \zeta)$ is non-degenerate and satisfies

$$
Q_{1}(\tau, \zeta) P_{0}(\tau, \zeta)=B_{1}(\tau, \zeta) P_{0}(\tau, \zeta)=P_{0}(\tau, \zeta) B_{1}(\tau, \zeta)=P_{0}(\tau, \zeta) Q_{1}(\tau, \zeta) .
$$

By the method prensented in Chapter 9 of Taylor [20], we obtain

LEMma 5.3. (i) There exists a pseudo-differential operator $\tilde{E} \in \Psi_{\text {phg }}^{0}\left(\mathbb{R} \times \Gamma ; \mathbb{C}, \mathbb{C}^{3}\right)$ such that

$$
\sigma_{p}(\tilde{E})(\tau, \zeta)=\left|e_{0}^{(e)}(\tau, \zeta)\right|^{-1} e_{0}^{(e)}(\tau, \zeta) \quad\left(\text { on } V_{1}\right), \quad \tilde{E}^{*} \tilde{E}=1 \bmod \Psi_{V_{1}, \tilde{V}_{1}}^{-\infty}(\mathbb{R} \times \Gamma) .
$$

(ii) Set $\tilde{P}_{0}=I-\tilde{E} \tilde{E}^{*}$. Then, $\tilde{P}_{0}$ becomes a pseudo-differential operator in $\Psi_{\text {phg }}^{0}(\mathbb{R} \times$ $\left.\Gamma ; \mathbb{C}^{3}, \mathbb{C}^{3}\right)$ and satisfies

$$
\sigma_{p}\left(\tilde{P}_{0}\right)=P_{0} \quad\left(\text { on } V_{1}\right), \quad \tilde{E}^{*} \tilde{P}_{0}=\tilde{P}_{0} \tilde{E}=0 \quad \bmod \quad \Psi_{V_{1}, \tilde{V}_{1}}^{-\infty}(\mathbb{R} \times \Gamma) .
$$


Proposition 5.4. (i) There exist a scalar-valued symbol $\lambda^{(e)}(\tau, \zeta) \in S_{p h g}^{1}\left(V_{1}\right)$ and a pseudo-differential operator $\tilde{E}^{(0)} \in \Psi_{\text {phg }}^{0}\left(\mathbb{R} \times \Gamma ; \mathbb{C}, \mathbb{C}^{3}\right)$ independent of $t$ such that $\tilde{E}^{(0)}-\tilde{E} \in \Psi_{\text {phg }}^{-1}\left(\mathbb{R} \times \Gamma ; \mathbb{C}, \mathbb{C}^{3}\right)$ and that

$$
\begin{aligned}
& B_{\chi_{1}} \tilde{E}^{(0)}=\tilde{E} O p\left(\chi_{1} \lambda^{(e)}\right) \quad \bmod \Psi_{W_{1}, \tilde{W}_{1}}^{-\infty}(\mathbb{R} \times \Gamma), \quad \sigma_{p}\left(\tilde{E}^{*}\right) \sigma_{p}\left(\tilde{E}^{(0)}-\tilde{E}\right)=0, \\
& \lambda^{(e)}(\tau, \zeta) \sim \lambda^{(e), 0}(\tau, \zeta)+\sum_{j=0}^{\infty} \lambda_{-j}^{(e)}(\tau, \zeta), \quad \check{I}_{\mathbb{R} \times \Gamma} \lambda^{(e)}(\tau, \zeta)=\overline{\lambda^{(e)}(\tau, \zeta)} \quad \text { on } V_{1},
\end{aligned}
$$

where each $\lambda_{-j}^{(e)}$ belongs to $S^{-j}\left(V_{1}\right)$ and is homogeneous of order $-j$.

(ii) There exist pseudo-differential operators $\tilde{Q} \in \Psi_{\text {phg }}^{-1}\left(\mathbb{R} \times \Gamma ; \mathbb{C}^{3}, \mathbb{C}^{3}\right)$ and $\tilde{F}^{(0)} \in$ $\Psi_{\text {phg }}^{0}\left(\mathbb{R} \times \Gamma ; \mathbb{C}^{3}, \mathbb{C}\right)$ such that

$$
\begin{array}{ll}
\sigma_{p}(\tilde{Q})(t, \tau, \zeta)=\chi_{1}(t, \tau, \zeta) Q_{1}(\tau, \zeta)^{-1} & \text { on } \tilde{W}_{1}, \\
\sigma_{p}\left(\tilde{F}^{(0)}\right)(t, \tau, \zeta) e_{0}^{(e)}(\tau, \zeta)=\frac{|\tau| \chi_{1}(t, \tau, \zeta)}{c_{R}\|\zeta\|_{\Gamma}}\left|e_{0}^{(e)}(\tau, \zeta)\right| & \text { on } T^{*}(\mathbb{R} \times \Gamma), \\
\sigma_{p}\left(\tilde{F}^{(0)}\right)(t, \tau, \zeta) P_{0}(\tau, \zeta)=0 & \text { on } T^{*}(\mathbb{R} \times \Gamma), \\
B_{\chi_{1}} \tilde{Q}=O p\left(\chi_{1}\right)-\tilde{E} \tilde{F}^{(0)} & \bmod \Psi_{W_{1}, \tilde{W}_{1}}^{-\infty}(\mathbb{R} \times \Gamma) .
\end{array}
$$

Proof. Since $B_{1} e_{0}^{(e)}(\tau, \zeta)=\lambda^{(e), 0} e_{0}^{(e)}(\tau, \zeta)$ on $T^{*}(\mathbb{R} \times \Gamma)$, there exists $\tilde{E}_{0} \in$ $\Psi_{\text {phg }}^{0}\left(\mathbb{R} \times \Gamma ; \mathbb{C}, \mathbb{C}^{3}\right)$ such that

$$
B_{\chi_{1}} \tilde{E}=\tilde{E} O p\left(\chi_{1} \lambda^{(e), 0}\right)+\tilde{E}_{0} .
$$

By Lemma 5.3 and (5.3), we get a scalar-valued symbol $\lambda_{0}^{(e)}(\tau, \zeta) \in S^{0}\left(T^{*}(\mathbb{R} \times \Gamma)\right)$ such that

$$
\sigma_{p}\left(\tilde{E}_{0}\right)(\tau, \zeta)=\sigma_{p}(\tilde{E}) \lambda_{0}^{(e)}(\tau, \zeta)+B_{1} P_{0} Q_{1}^{-1} \sigma_{p}\left(\tilde{E}_{0}\right)(\tau, \zeta) \quad \text { on } V_{1} .
$$

We take a cutoff function $\tilde{\chi}_{1}(t, \tau, \zeta) \in C^{\infty}\left(T^{*}(\mathbb{R} \times \Gamma)\right)$ such that $0 \leq \tilde{\chi}_{1} \leq 1$, $\operatorname{supp}\left[\tilde{\chi}_{1}\right] \subset \tilde{W}_{1}, \tilde{\chi}_{1}(t, \tau, \zeta)=1$ on $W_{1}$ and $\tilde{\chi}_{1}(t, \tau, \zeta)=0$ on a neighborhood of the zero section of $T^{*}(\mathbb{R} \times \Gamma)$, and set $\tilde{E}_{-1}=O p\left(\chi_{1}\right) \tilde{E}_{0}-\tilde{E} O p\left(\chi_{1} \lambda_{0}^{(e)}\right)-$ $B_{\chi_{1}} O p\left(\tilde{\chi}_{1} P_{0} Q_{1}^{-1} \sigma_{p}\left(\tilde{E}_{0}\right)\right)$. Then, using (5.4) and (5.5), we see that $\tilde{E}_{-1} \in \Psi_{p h g}^{-1}(\mathbb{R} \times$ $\left.\Gamma ; \mathbb{C}, \mathbb{C}^{3}\right)$ and

$$
B_{\chi_{1}}\left(\tilde{E}-O p\left(\tilde{\chi}_{1} P_{0} Q_{1}^{-1} \sigma_{p}\left(\tilde{E}_{0}\right)\right)\right)=\tilde{E} O p\left(\chi_{1}\left(\lambda^{(e), 0}+\lambda_{0}^{(e)}\right)\right)+\tilde{E}_{-1}+\left(I-O p\left(\chi_{1}\right)\right) \tilde{E}_{0} .
$$

Furthermore, there exists a scalar-valued symbol $\lambda_{-1}^{(e)}(\tau, \zeta) \in S^{-1}\left(V_{1}\right)$ such that $\sigma_{p}\left(\tilde{E}_{-1}\right)(\tau, \zeta)=\sigma_{p}(\tilde{E}) \lambda_{-1}^{(e)}(\tau, \zeta)+B_{1} P_{0} Q_{1}^{-1} \sigma_{p}\left(\tilde{E}_{-1}\right)(\tau, \zeta)$. Next we set

$\tilde{E}_{-2}=O p\left(\chi_{1}\right) \tilde{E}_{-1}-\tilde{E} O p\left(\chi_{1} \lambda_{-1}^{(e)}\right)-B_{\chi_{1}} O p\left(\tilde{\chi}_{1} P_{0} Q_{1}^{-1} \sigma_{p}\left(\tilde{E}_{-1}\right)\right) \in \Psi_{p h g}^{-2}\left(\mathbb{R} \times \Gamma ; \mathbb{C}, \mathbb{C}^{3}\right)$.

We then obtain

$$
\begin{aligned}
B_{\chi_{1}}\left(\tilde{E}-O p\left(\tilde{\chi}_{1} P_{0} Q_{1}^{-1} \sigma_{p}\left(\tilde{E}_{0}\right)\right)-O p\left(\tilde{\chi}_{1} P_{0} Q_{1}^{-1} \sigma_{p}\left(\tilde{E}_{-1}\right)\right)\right) \\
=\tilde{E} O p\left(\chi_{1}\left(\lambda^{(e), 0}+\lambda_{0}^{(e)}+\lambda_{-1}^{(e)}\right)\right)+\tilde{E}_{-2}+\left(I-O p\left(\chi_{1}\right)\right)\left(\tilde{E}_{0}+\tilde{E}_{-1}\right) .
\end{aligned}
$$


Here, note that $\check{I}_{\mathbb{R} \times \Gamma} \lambda_{j}^{(e)}(\tau, \zeta)=\overline{\lambda_{j}^{(e)}(\tau, \zeta)}(j=0,1)$, which follows from (iii) in Proposition 5.1. Noting that ess.supp $\left(I-O p\left(\chi_{1}\right)\right) \cap \tilde{W}_{1}=\emptyset$ and repeating these procedures, we obtain (i) in Proposition 5.4. It is also seen that $\sigma_{p}\left(\tilde{E}^{*}\right) \sigma_{p}\left(\tilde{E}^{(0)}-\tilde{E}\right)=$ 0 since the equalities $\sigma_{p}\left(\tilde{E}^{*}\right) \sigma_{p}\left(O p\left(\tilde{\chi}_{1} P_{0} Q_{1}^{-1} \sigma_{p}\left(\tilde{E}_{j}\right)\right)\right)=0(j=0,1,2, \ldots)$ follow from $\sigma_{p}\left(\tilde{E}^{*}\right) P_{0}=\left|e^{(0)}\right|^{-1}\left(e^{(0)}\right)^{*} P_{0}=0$.

We see from Lemma 5.3 that $\sigma_{p}\left(B_{\chi_{1}}+\tilde{E} O p\left(|\tau| \chi_{1} \tilde{\lambda}^{(e), 0}\right) \tilde{E}^{*}\right)=\chi_{1}(t, \tau, \eta) Q_{1}(\tau, \eta)$ on $\tilde{W}_{1}$. Therefore, noting that $Q_{1}(\tau, \eta)$ is non-degenerate on $V_{0}^{\prime}$, we have a pseudodifferential operator $\tilde{Q} \in \Psi_{p h g}^{-1}\left(\mathbb{R} \times \Gamma ; \mathbb{C}^{3}, \mathbb{C}^{3}\right)$ such that $\sigma_{p}(\tilde{Q})(t, \tau, \eta)=\chi_{1}(t, \tau, \eta)$ $Q_{1}(\tau, \eta)^{-1}$ and

$$
\left(B_{\chi_{1}}+\tilde{E} O p\left(|\tau| \chi_{1} \tilde{\lambda}^{(e), 0}\right) \tilde{E}^{*}\right) \tilde{Q}=O p\left(\chi_{1}\right) \quad \bmod \quad \Psi_{W_{1}, \tilde{W}_{1}}^{-\infty}(\mathbb{R} \times \Gamma) .
$$

We put $\tilde{F}^{(0)}=O p\left(|\tau| \chi_{1} \tilde{\lambda}^{(e), 0}\right) \tilde{E}^{*} \tilde{Q}$. Since ess.supp $\left(I-O p\left(\chi_{1}\right)\right) \cap \tilde{W}_{1}=\emptyset$ and $Q_{1}^{-1}(\tau, \zeta) e_{0}^{(e)}(\tau, \zeta)=\left(c_{R}\|\zeta\|_{\Gamma} \tilde{\lambda}^{(e), 0}(\tau, \zeta)\right)^{-1} e_{0}^{(e)}(\tau, \zeta)$, it follows that $\tilde{F}^{(0)} \in$ $\Psi_{p h g}^{0}\left(\mathbb{R} \times \Gamma ; \mathbb{C}^{3}, \mathbb{C}\right), \sigma_{p}\left(\tilde{F}^{(0)}\right)=|\tau| \chi_{1} \tilde{\lambda}^{(e), 0} \sigma_{p}\left(\tilde{E}^{*}\right) Q_{1}^{-1}$ and $\sigma_{p}\left(\tilde{F}^{(0)}\right)(t, \tau, \zeta) e_{0}^{(e)}(\tau, \zeta)=$ $c_{R}^{-1}\|\zeta\|_{\Gamma}^{-1}|\tau| \chi_{1}(t, \tau, \zeta)\left|e_{0}^{(e)}(\tau, \zeta)\right|$. Hence, (ii) of Proposition 5.4 is obtained. The proof is complete.

The next step is to reduce the construction of the solution $v_{\varphi}^{R, b}$ to a problem given by a scalar-valued pseudo-differential equation of the real principal type (cf. (5.7)). Before this, we prepare the following lemma used for error estimation.

Lemma 5.5. Let $P_{j} \in \Psi^{m_{j}}(\mathbb{R} \times \Gamma)(j=1,2), \gamma \in C_{0}^{\infty}\left(\partial \mathbb{R}_{+}^{3}\right)$, and $\eta \in C_{0}^{\infty}(\mathbb{R})$. Then, for any $s \in \mathbb{R}$ there is a constant $C>0$ such that

$$
\left\|\eta P_{1} \gamma P_{2} \tilde{\varphi}_{\theta}\right\|_{H^{s}\left(\mathbb{R} \times \partial \mathbb{R}_{+}^{3}\right)} \leq C\|\varphi\|_{H^{m_{1}+m_{2}+s}(\mathbb{R})}, \quad \varphi \in C_{0}^{\infty}(I) .
$$

Proof of Lemma 5.5. Take a cutoff function $\tilde{\gamma}\left(x^{\prime}\right) \in C_{0}^{\infty}\left(\partial \mathbb{R}_{+}^{3}\right)$ that satisfies $\tilde{\gamma}\left(x^{\prime}\right)=1$ in a neighborhood of $\operatorname{supp}[\gamma]$. Since $\eta P_{1} \gamma P_{2} \in \Psi^{m_{1}+m_{2}}(\mathbb{R} \times \Gamma)$ and $\left(\tilde{\gamma} \tilde{\varphi}_{\theta}\right)^{\wedge}(\tau, \xi)=\hat{\varphi}(-\tau) \tilde{\tilde{\gamma}}\left(\xi+\tau c_{R}^{-1} \theta\right)$, it follows that

$$
\left\|\eta P_{1} \gamma P_{2} \tilde{\gamma} \tilde{\varphi}_{\theta}\right\|_{H^{s}\left(\mathbb{R} \times \partial \mathbb{R}_{+}^{3}\right)} \leq C\left\|\tilde{\gamma} \tilde{\varphi}_{\theta}\right\|_{H^{m_{1}+m_{2}+s}\left(\mathbb{R} \times \partial \mathbb{R}_{+}^{3}\right)} \leq C^{\prime}\|\varphi\|_{H^{m_{1}+m_{2}+s}(\mathbb{R})} .
$$

Noting that $\operatorname{supp}[\gamma] \cap \operatorname{supp}[(1-\tilde{\gamma})]=\emptyset$ and $\gamma \in C_{0}^{\infty}\left(\partial \mathbb{R}_{+}^{3}\right)$, we obtain $\eta P_{1} \gamma P_{2}(I-$ $\tilde{\gamma})\left\langle x^{\prime}\right\rangle^{l} \in \Psi^{-\infty}(\mathbb{R} \times \Gamma)$. Therefore, we have

$$
\left\|\eta P_{1} \gamma P_{2}(I-\tilde{\gamma}) \tilde{\varphi}_{\theta}\right\|_{H^{s}\left(\mathbb{R} \times \partial \mathbb{R}_{+}^{3}\right)} \leq C\left\|\langle\cdot\rangle^{-l} \tilde{\varphi}_{\theta}\right\|_{H^{-s^{\prime}}\left(\mathbb{R} \times \partial \mathbb{R}_{+}^{3}\right)}
$$

for $s^{\prime} \geq 0$ and $l \geq 2$. Combining this with $\left\|\langle\cdot\rangle^{-l} \tilde{\varphi}_{\theta}\right\|_{H^{-s^{\prime}}\left(\mathbb{R} \times \partial \mathbb{R}_{+}^{3}\right)} \leq C_{l, s^{\prime}}\|\varphi\|_{H^{-s^{\prime}}(\mathbb{R})}$, we obtain $\left\|\eta P_{1} \gamma P_{2}(I-\tilde{\gamma}) \tilde{\varphi}_{\theta}\right\|_{H^{s}\left(\mathbb{R} \times \partial \mathbb{R}_{+}^{3}\right)} \leq C_{s, s^{\prime}}\|\varphi\|_{H^{s^{\prime}}(\mathbb{R})}$. Thus, Lemma 5.5 is obtained.

Now we begin to reduce the problem. Take a conic neighborhood $V_{2}$ of $\mathcal{R}_{\Gamma}$ such that $V_{2} \subset \subset V_{1}$, and set $W_{2}=\left\{(t, \tau, \zeta) \in V_{2} ;|t|<t_{1}+5 c_{R}^{-1}\right\}$. Furthermore, take a cutoff function $\chi_{2}(t, \tau, \zeta) \in C^{\infty}\left(T^{*}(\mathbb{R} \times \Gamma)\right)$ satisfying $0 \leq \chi_{2} \leq 1$, supp $\left[\chi_{2}\right] \subset W_{1}$, $\chi_{2}(t, \tau, \zeta)=1$ on $W_{2}$ and $\chi_{2}(t, \tau, \zeta)=0$ near the zero section in $T^{*}(\mathbb{R} \times \Gamma)$. Assume that there exists a $\mathcal{B}^{\infty}\left(I_{t_{1}+5 c_{R}^{-1}}\left(s_{0}\right) \times \Gamma\right)$-valued linear mapping $k_{\varphi}\left(t, x^{\prime} ; \theta\right)$ on $C_{0}^{\infty}(I)$ such that

$$
\begin{aligned}
O p\left(\chi_{1} \lambda^{(e)}\right) k_{\varphi}\left(t, x^{\prime} ; \theta\right) \simeq \tilde{F}^{(0)} O p\left(\chi_{2}\right) \psi_{2} F_{1} \tilde{\varphi}_{\theta}\left(t, x^{\prime}\right) & \\
& \text { with respect to } C_{0}^{\infty}(I) \rightarrow \mathcal{B}^{\infty}\left(I_{t_{1}+5 c_{R}^{-1}}\left(s_{0}\right) \times \Gamma\right)
\end{aligned}
$$


which is proved later.

Using Proposition 5.4, we can construct the boundary value $\left.v_{\varphi}^{R, b}\left(t, x^{\prime} ; \theta\right)\right|_{\mathbb{R} \times \Gamma}$. This means that the required $v_{\varphi}^{R, b}(t, x ; \theta)$ is obtained by applying $\mathcal{P}^{-} O p\left(\chi_{2}\right)$ to the boundary value. We show here the construction of $\left.v_{\varphi}^{R, b}\left(t, x^{\prime} ; \theta\right)\right|_{\mathbb{R} \times \Gamma}$. Set

$$
g_{\varphi}\left(t, x^{\prime} ; \theta\right)=\tilde{Q} O p\left(\chi_{2}\right) \psi_{2} F_{1} \tilde{\varphi}_{\theta}\left(t, x^{\prime}\right)+\tilde{E}^{(0)} k_{\varphi}\left(t, x^{\prime} ; \theta\right) .
$$

Then, we obtain

$$
\begin{aligned}
B_{\chi_{1}} g_{\varphi}\left(t, x^{\prime} ; \theta\right)= & O p\left(\chi_{2}\right) \psi_{2} F_{1} \tilde{\varphi}_{\theta}\left(t, x^{\prime}\right)+\left(O p\left(\chi_{1}\right)-I\right) O p\left(\chi_{2}\right) \psi_{2} F_{1} \tilde{\varphi}_{\theta}\left(t, x^{\prime}\right) \\
& +R_{-\infty, 1} O p\left(\chi_{2}\right) \psi_{2} F_{1} \tilde{\varphi}_{\theta}\left(t, x^{\prime}\right)+R_{-\infty, 2} k_{\varphi}\left(t, x^{\prime} ; \theta\right)+R_{-\infty, 3} \tilde{\varphi}_{\theta}\left(t, x^{\prime}\right),
\end{aligned}
$$

where $R_{-\infty, 1}=B_{\chi_{1}} \tilde{Q}-O p\left(\chi_{1}\right)-\tilde{E} \tilde{F}^{(0)}, R_{-\infty, 2}=B_{\chi_{1}} \tilde{E}^{(0)}-\tilde{E} O p\left(\chi_{1} \lambda^{(e)}\right)$ and $R_{-\infty, 3} \tilde{\varphi}_{\theta}=\tilde{E}\left\{O p\left(\chi_{1} \lambda^{(e)}\right) k_{\varphi}-\tilde{F}^{(0)} O p\left(\chi_{2}\right) \psi_{2} F_{1} \tilde{\varphi}_{\theta}\right\}$. We have $R_{-\infty, j} O p\left(\chi_{2}\right)(j=1,2)$ $\in \Psi^{-\infty}(\mathbb{R} \times \Gamma)$ since ess.supp $O p\left(\chi_{2}\right) \subset W_{1}$.

By Lemma 5.5, we obtain $R_{-\infty, 1} O p\left(\chi_{2}\right) \psi_{2} F_{1} \tilde{\varphi}_{\theta} \simeq 0$ with respect to $C_{0}^{\infty}(I) \rightarrow$ $\mathcal{B}^{\infty}\left(I_{t_{1}+4 c_{R}^{-1}}\left(s_{0}\right) \times \Gamma\right)$. It follows from $\operatorname{supp}\left[\chi_{2}\right] \cap \operatorname{supp}\left[\left(\chi_{1}-1\right)\right]=\emptyset$ that $O p\left(\chi_{2}\right)\left(O p\left(\chi_{1}\right)-I\right) \psi_{2} F_{1} \tilde{\varphi}_{\theta} \simeq 0$ with respect to $C_{0}^{\infty}(I) \rightarrow \mathcal{B}^{\infty}\left(I_{t_{1}+4 c_{R}^{-1}}\left(s_{0}\right) \times \Gamma\right)$. Furthermore, the ellipticity of $\lambda^{(e)}$ on $V_{1} \backslash \mathcal{R}_{\Gamma}$ yields that $\left(I-O p\left(\chi_{2}\right)\right) k_{\varphi} \simeq 0$ with respect to $C_{0}^{\infty}(I) \rightarrow \mathcal{B}^{\infty}\left(I_{t_{1}+4 c_{R}^{-1}}\left(s_{0}\right) \times \Gamma\right)$ since $k_{\varphi}$ satisfies the equation (5.7). From the form of $k_{\varphi}$ that is constructed later, we can also see that $R_{-\infty, 2} O p\left(\chi_{2}\right) k_{\varphi} \simeq$ 0 with respect to $C_{0}^{\infty}(I) \rightarrow \mathcal{B}^{\infty}\left(I_{t_{1}+4 c_{R}^{-1}}\left(s_{0}\right) \times \Gamma\right)$ (cf. Proposition 5.6). It follows from (5.7) and Lemma 5.5 that $R_{-\infty, 3} \tilde{\varphi}_{\theta} \simeq 0$ with respect to $C_{0}^{\infty}(I) \rightarrow$ $\mathcal{B}^{\infty}\left(I_{t_{1}+4 c_{R}^{-1}}\left(s_{0}\right) \times \Gamma\right)$. Thus, we have $B_{\chi_{1}} g_{\varphi} \simeq O p\left(\chi_{2}\right) \psi_{2} F_{1} \tilde{\varphi}_{\theta}$ with respect to $C_{0}^{\infty}(I)$ $\rightarrow \mathcal{B}^{\infty}\left(I_{t_{1}+4 c_{R}^{-1}}\left(s_{0}\right) \times \Gamma\right)$. Therefore, by (ii) of Proposition 4.5 we can put $v_{\varphi}^{R, b}(t, x ; \theta)=$ $\left(\mathcal{P}^{-} O p\left(\chi_{1}\right) g_{\varphi}(\cdot, \cdot ; \theta)\right)(t, x)$ and obtain

$$
\begin{aligned}
\left.v_{\varphi}^{R, b}\left(t, x^{\prime} ; \theta\right)\right|_{\mathbb{R} \times \Gamma} & \simeq O p\left(\chi_{1}\right) g_{\varphi}\left(t, x^{\prime} ; \theta\right) \\
& \text { with respect to } C_{0}^{\infty}(I) \rightarrow \mathcal{B}^{\infty}\left(I_{t_{1}+3 c_{R}^{-1}}\left(s_{0}\right) \times \Gamma\right) .
\end{aligned}
$$

Thus, we have reduced the construction of the solution $v_{\varphi}^{R, b}$ to solving (5.7).

The final step is to construct the asymptotic solution $k_{\varphi}\left(t, x^{\prime} ; \theta\right)$ of the equation (5.7). As is used in Hörmander [2], $C_{0}^{\infty}\left(\mathbb{R} \times \Gamma ; \Omega^{\frac{1}{2}} \otimes \mathbb{C}^{m}\right)$ denotes the space of smooth sections of $\Omega^{\frac{1}{2}} \otimes \mathbb{C}^{m}$ with compact support, where $\Omega^{\frac{1}{2}}$ is the half-density bundle on $\mathbb{R} \times \Gamma$. We denote the half-density on $\mathbb{R} \times \Gamma$, which is defined by the standard Riemann metric on $\mathbb{R} \times \Gamma$, by $d V_{\mathbb{R} \times \Gamma}^{\frac{1}{2}}$. Note that any section in $C_{0}^{\infty}\left(\mathbb{R} \times \Gamma ; \Omega^{\frac{1}{2}} \otimes \mathbb{C}^{m}\right)$ can be expressed by multiplying $d V_{\mathbb{R} \times \Gamma}^{\frac{1}{2}}$ to functions in $C_{0}^{\infty}\left(\mathbb{R} \times \Gamma ; \mathbb{C}^{m}\right)$. For pseudodifferential operators $P \in \Psi^{l}\left(\mathbb{R} \times \Gamma ; \mathbb{C}^{m_{1}}, \mathbb{C}^{m_{2}}\right)$, we employ the pseudo-differential operators $P_{\Omega^{\frac{1}{2}}}$ from $C_{0}^{\infty}\left(\mathbb{R} \times \Gamma ; \Omega^{\frac{1}{2}} \otimes \mathbb{C}^{m_{1}}\right)$ to $C_{0}^{\infty}\left(\mathbb{R} \times \Gamma ; \Omega^{\frac{1}{2}} \otimes \mathbb{C}^{m_{2}}\right)$ :

$$
P_{\Omega^{\frac{1}{2}}}\left(f d V_{\mathbb{R} \times \Gamma}^{\frac{1}{2}}\right)=(P f) d V_{\mathbb{R} \times \Gamma}^{\frac{1}{2}}, \quad f \in C_{0}^{\infty}\left(\mathbb{R} \times \Gamma ; \mathbb{C}^{m_{1}}\right) .
$$

Note that the space $\Psi^{l}\left(\mathbb{R} \times \Gamma ; \Omega^{\frac{1}{2}} \otimes \mathbb{C}^{m_{1}}, \Omega^{\frac{1}{2}} \otimes \mathbb{C}^{m_{2}}\right)$ consists of these operators $P_{\Omega^{\frac{1}{2}}}$. We simply write $\Psi^{l}\left(\mathbb{R} \times \Gamma ; \Omega^{\frac{1}{2}}, \Omega^{\frac{1}{2}}\right)$ if $m_{1}=m_{2}=1$. For pseudo-differential operators $Q \in \Psi^{l}\left(\mathbb{R} \times \Gamma ; \Omega^{\frac{1}{2}}, \Omega^{\frac{1}{2}}\right), \sigma_{p}(Q)$ and $\sigma_{s p}(Q)$ denote the principal symbol and the subprincipal symbol, respectively. 
Take a pseudo-differential operator $A_{0} \in \Psi^{0}\left(\mathbb{R} \times \Gamma ; \Omega^{\frac{1}{2}}, \Omega^{\frac{1}{2}}\right)$ such that

$$
\sigma_{p}\left(A_{0}\right)(t, \tau, \zeta)=\left(\tilde{\lambda}_{0}^{(e), 0}(\tau, \zeta)\right)^{-1}, \quad \sigma_{s p}\left(A_{0}\right)(t, \tau, \zeta)=0 .
$$

Then, we obtain $A_{0} O p\left(\chi_{1} \lambda^{(e)}\right)_{\Omega^{\frac{1}{2}}} \in \Psi^{1}\left(\mathbb{R} \times \Gamma ; \Omega^{\frac{1}{2}}, \Omega^{\frac{1}{2}}\right)$ and

$$
\left\{\begin{array}{c}
\sigma_{p}\left(A_{0} O p\left(\chi_{1} \lambda^{(e)}\right)_{\Omega^{\frac{1}{2}}}\right)(t, \tau, \zeta)=\chi_{1}(t, \tau, \zeta)\left(\tilde{c}_{R}\left(x^{\prime}\right)\|\zeta\|_{\Gamma}-|\tau|\right) \\
\text { on } T^{*}(\mathbb{R} \times \Gamma) \\
\sigma_{s p}\left(A_{0} O p\left(\chi_{1} \lambda^{(e)}\right)_{\Omega^{\frac{1}{2}}}\right)(t, \tau, \zeta)=\sigma_{s p}\left(O p\left(\chi_{1} \lambda^{(e)}\right)_{\Omega^{\frac{1}{2}}}\right)(t, \tau, \zeta) \\
\text { on }\left\{(t, \tau, \zeta) \in T^{*}\left(\mathbb{R}^{\times} \times \Gamma\right) ; \chi_{1}(t, \tau, \zeta)=1\right\}
\end{array}\right.
$$

Note that $\tilde{F}^{(0)} O p\left(\chi_{2}\right) \psi_{2} F_{1}$ can be regarded as a pseudo-differential operator on $\mathbb{R} \times$ $\partial \mathbb{R}_{+}^{3}$ since supp $\left[\psi_{2}\right] \subset B_{r_{0}+2} \backslash B_{r_{0}+1}$. We take $\phi_{5}(s) \in C_{0}^{\infty}\left(\left(s_{0}-2 \varepsilon, s_{0}+2 \varepsilon\right)\right)$ satisfying $\phi_{5}(s)=1$ on $\left[s_{0}-\varepsilon, s_{0}+\varepsilon\right]$, and set $\left(\tilde{\phi}_{5}\right)_{\theta}\left(t, x^{\prime}\right)=\phi_{5}\left(c_{R}^{-1} \theta \cdot x^{\prime}-t\right)$. Noting that $\left(\tilde{\varphi}_{\theta}\right)^{\wedge}(\tau, \xi)=(2 \pi)^{2} \delta\left(\xi+\tau c_{R}^{-1} \theta\right) \hat{\varphi}(-\tau)$ and using Proposition 5.4 and Proposition 4.5, we can rewrite the equation (5.7) as follows:

$$
\begin{aligned}
& A_{0} O p\left(\chi_{1} \lambda^{(e)}\right)_{\Omega^{\frac{1}{2}}} \tilde{k}_{\varphi}\left(t, x^{\prime} ; \theta\right) \\
& \simeq\left((2 \pi)^{-1} \int_{\mathbb{R}} e^{i \tau\left(c_{R}^{-1} \theta \cdot x^{\prime}-t\right)}\left(\tilde{\phi}_{5}\right)_{\theta}\left(t, x^{\prime}\right) a\left(t, x^{\prime}, \tau\right) \hat{\varphi}(\tau) d \tau\right) d V_{\mathbb{R} \times \Gamma}^{\frac{1}{2}} \\
& \text { with respect to } C_{0}^{\infty}(I) \rightarrow \mathcal{B}^{\infty}\left(I_{t_{1}+5 c_{R}^{-1}}\left(s_{0}\right) \times \Gamma ; \Omega^{\frac{1}{2}}\right),
\end{aligned}
$$

where $a \in S_{\text {phg }}^{1}\left(\mathbb{R}_{t} \times \mathbb{R}_{x^{\prime}}^{2} \times \mathbb{R}_{\tau}\right)$ satisfies

$$
\operatorname{supp}[a] \subset\left[-\left(s_{0}+t_{1}+6 c_{R}^{-1}\right),-s_{0}+t_{1}+6 c_{R}^{-1}\right] \times \overline{\left(B_{r_{0}+2} \backslash B_{r_{0}+1}\right)} \times \mathbb{R}_{\tau}
$$

and has an asymptotic expansion $a \sim \sum_{j=0}^{\infty} a_{1-j}$ with

$$
a_{1}\left(t, x^{\prime}, \tau\right)=\alpha^{(1)}|\tau| \chi_{2}\left(t,-\tau, x^{\prime}, \frac{\tau}{c_{R}} \theta\right) \theta \cdot \nabla_{x^{\prime}} \tilde{\psi}\left(x^{\prime}\right)
$$

$\left(\alpha^{(1)}=c_{R}^{3} c_{S}^{-2} C_{0}^{R} \sqrt{2 \pi \rho_{0} \xi_{R}^{(1)}\left(\xi_{R}^{(1)}+\xi_{R}^{(2)}\right)}\right)$. Namely, the solution $k_{\varphi}\left(t, x^{\prime} ; \theta\right)$ of $(5.7)$ is obtained from $\tilde{k}_{\varphi}\left(t, x^{\prime} ; \theta\right)$ as follows:

$$
k_{\varphi}\left(t, x^{\prime} ; \theta\right) d V_{\mathbb{R} \times \Gamma}^{\frac{1}{2}}=\tilde{k}_{\varphi}\left(t, x^{\prime} ; \theta\right) .
$$

We now construct $\tilde{k}_{\varphi}\left(t, x^{\prime} ; \theta\right)$. For $\theta={ }^{t}\left(\theta_{1}, \theta_{2}\right) \in S^{1}$, we put $\theta^{\perp}={ }^{t}\left(\theta_{2},-\theta_{1}\right)$. We set $L_{r_{0}}^{ \pm}=\left\{x^{\prime} \in \partial \mathbb{R}_{+}^{3} ; \theta \cdot x^{\prime}= \pm\left(r_{0}+3 / 2\right),\left|\theta^{\perp} \cdot x^{\prime}\right| \leq r_{0}+1 / 2\right\}, L_{r_{0}}^{0}=\left\{x^{\prime} \in\right.$ $\left.\partial \mathbb{R}_{+}^{3} ; \theta \cdot x^{\prime}=r_{0}+3 / 2, r_{0}+1 / 2<\left|\theta^{\perp} \cdot x^{\prime}\right| \leq r_{0}+2\right\}$. For any $x_{0}^{\prime} \in L_{r_{0}}^{ \pm}$, we take a neighborhood $U_{x_{0}^{\prime}}=\left\{x^{\prime} \in \partial \mathbb{R}_{+}^{3} ;\left|\theta \cdot\left(x^{\prime}-x_{0}^{\prime}\right)\right|<\delta+1 / 2,\left|\theta^{\perp} \cdot\left(x^{\prime}-x_{0}^{\prime}\right)\right|<\delta\right\}$, and for $x_{1}^{\prime} \in L_{r_{0}}^{0}, U_{x_{1}^{\prime}}=\left\{x^{\prime} \in \partial \mathbb{R}_{+}^{3} ;\left|\theta \cdot x^{\prime}\right|<r_{0}+2+\delta,\left|\theta^{\perp} \cdot\left(x^{\prime}-x_{1}^{\prime}\right)\right|<\delta\right\}$, where $\delta>0$ is chosen to be sufficiently small. Let $T=c_{R}^{-1}\left(r_{0}+3 / 2\right)+t_{1}+8 c_{R}^{-1}$, and set

$\mathcal{L}^{2, \pm}\left(U_{x_{0}^{\prime}}\right)=\left\{\left(x^{\prime}, \pm c_{R}^{-1} \theta\right) \in T^{*}(\Gamma) ; x^{\prime} \in U_{x_{0}^{\prime}}\right\}$,

$\mathcal{L}_{T}^{3, \pm}\left(U_{x_{0}^{\prime}}\right)=\left\{\left(s, \mp H\left(h_{ \pm}^{s-\tau_{0}}(\zeta)\right), h_{ \pm}^{s-\tau_{0}}(\zeta)\right) \in T^{*}(\mathbb{R} \times \Gamma) ;\left|t-\tau_{0}\right|<T, \zeta \in \mathcal{L}^{2, \pm}\left(U_{x_{0}^{\prime}}\right)\right\}$,

where $\tau_{0}=c_{R}^{-1}\left(r_{0}+3 / 2\right)-s_{0}$ and $h_{ \pm}^{s}(\zeta)\left(=h_{\mp}^{-s}(\zeta)\right)$ is the phase flow of the Hamiltonian $\pm H(\zeta)= \pm \tilde{c}_{R}\left(x^{\prime}\right)\|\zeta\|_{\Gamma}$ with $h_{ \pm}^{0}(\zeta)=\zeta$. Note that $\mathcal{L}_{T}^{3, \pm}\left(U_{x_{0}^{\prime}}\right)$ are Lagrangian manifolds in $T^{*}(\mathbb{R} \times \Gamma)$, and that $h_{ \pm}^{s}$ satisfies

$$
\check{I}_{\Gamma}\left(h_{-}^{t}\left(y^{\prime},-c_{R}^{-1} \theta\right)\right)=h_{+}^{t}\left(y^{\prime}, c_{R}^{-1} \theta\right), \quad t \in \mathbb{R}, y^{\prime} \in U_{x_{0}^{\prime}},
$$


(where $\left.\check{I}_{\Gamma}: \zeta=\left(x^{\prime}, \zeta_{x^{\prime}}\right) \mapsto\left(x^{\prime},-\zeta_{x^{\prime}}\right)\right)$.

It follows from (1.2) that if $t_{1}(>0)$ chosen in section 2 is sufficiently large, then

$$
\begin{aligned}
& \left|\pi_{\Gamma}\left(h_{ \pm}^{t-\tau_{0}}\left(x^{\prime}, \pm c_{R}^{-1} \theta\right)\right)\right| \geq r_{0}+1 \\
& \quad t \leq-s_{0}-t_{1}, x^{\prime} \in U_{x_{0}^{\prime}}, x_{0}^{\prime} \in L_{r_{0}}^{ \pm} \cup L_{r_{0}}^{0}
\end{aligned}
$$

where $\pi_{\Gamma}: T^{*}(\Gamma) \rightarrow \Gamma$ is the projection to the base space $\Gamma$. Since $H(\zeta)=c_{R}|\xi|$ with $\zeta={ }^{t}\left(x^{\prime}, \xi\right) \in T^{*}\left(\partial \mathbb{R}_{+}^{3}\right)$ if $\left|\pi_{\Gamma}(\zeta)\right| \geq r_{0}$, the set $\left\{(t, \tau, \zeta) \in \mathcal{L}_{T}^{3, \pm}\left(U_{x_{0}^{\prime}}\right) ; t \geq \tau_{0}\right\}$ and $\tilde{\mathcal{L}}_{T}^{3, \pm}$ $\left(U_{x_{0}^{\prime}}\right)=\left\{(t, \tau, \zeta) \in \mathcal{L}_{T}^{3, \pm}\left(U_{x_{0}^{\prime}}\right) ; t \leq \tau_{0}-\left(s_{0}+t_{1}\right)\right\}$ are of the forms

$$
\begin{gathered}
\left\{(t, \tau, \zeta) \in \mathcal{L}_{T}^{3, \pm}\left(U_{x_{0}^{\prime}}\right) ; t \geq \tau_{0}\right\}=\left\{\left(t, \mp 1, x^{\prime}+c_{R}\left(t-\tau_{0}\right) \theta, \pm c_{R}{ }^{-1} \theta\right) ;\right. \\
\left.x^{\prime} \in U_{x_{0}^{\prime}}, \tau_{0} \leq t \leq \tau_{0}+T\right\}, \\
\tilde{\mathcal{L}}_{T}^{3, \pm}\left(U_{x_{0}^{\prime}}\right)=\left\{\left(\tau_{0}-\left(s_{0}+t_{1}\right)+\tau, \mp 1, z_{x^{\prime}}+c_{R} \tau \theta_{x^{\prime}}, \pm c_{R}{ }^{-1} \theta_{x^{\prime}}\right)\right. \\
\left.x^{\prime} \in U_{x_{0}^{\prime}},-8 \leq \tau \leq 0\right\},
\end{gathered}
$$

where $\left(z_{x^{\prime}}, \theta_{x^{\prime}}\right)$ is defined by $h^{-\left(s_{0}+t_{1}\right)-\tau_{0}}\left(x^{\prime}, \pm c_{R}{ }^{-1} \theta\right)=\left(z_{x^{\prime}}, \pm c_{R}{ }^{-1} \theta_{x^{\prime}}\right)$. If $U_{x_{0}^{\prime}}$ is small enough (i.e. $\delta>0$ is small enough), we can make the Maslov canonical operator $K_{x_{0}^{\prime}}^{ \pm}$(with the parameter $\tau$ ) from $C_{0}^{\infty}\left(\mathcal{L}_{T}^{3, \pm}\left(U_{x_{0}^{\prime}}\right)\right)$ to $C_{0}^{\infty}\left(\mathbb{R} \times \Gamma ; \Omega^{\frac{1}{2}}\right)$, which is used to construct the asymptotic solution $k_{\varphi}(t, x ; \theta)$. We breafly explain the construction of $K_{x_{0}^{\prime}}^{ \pm}$here.

We choose an open set $\tilde{U}_{x_{0}^{\prime}}$ with $\overline{U_{x_{0}^{\prime}}} \subset \tilde{U}_{x_{0}^{\prime}}$, and define $\mathcal{L}_{T}^{3, \pm}\left(\tilde{U}_{x_{0}^{\prime}}\right)$ and $\tilde{\mathcal{L}}_{T}^{3, \pm}\left(\tilde{U}_{x_{0}^{\prime}}\right)$ in the same way as $\mathcal{L}_{T}^{3, \pm}\left(U_{x_{0}^{\prime}}\right)$ and $\tilde{\mathcal{L}}_{T}^{3, \pm}\left(U_{x_{0}^{\prime}}\right)$, respectively. We put

$$
r^{ \pm}\left(t, y^{\prime}\right)=\left(t, \mp H\left(h_{ \pm}^{t-\tau_{0}}\left(y^{\prime}, \pm c_{R}^{-1} \theta\right)\right), h_{ \pm}^{t-\tau_{0}}\left(y^{\prime}, \pm c_{R}^{-1} \theta\right)\right) \quad\left(\left(t, y^{\prime}\right) \in \mathbb{R} \times \tilde{U}_{x_{0}^{\prime}}\right)
$$

We can choose connected open sets $\mathcal{O}_{j}^{ \pm} \subset \mathcal{L}_{T}^{3, \pm}\left(\tilde{U}_{x_{0}^{\prime}}\right)\left(j=0,1, \ldots, N_{x_{0}^{\prime}}\right)$ with following properties:

(i) The closure of $\mathcal{L}_{T}^{3, \pm}\left(U_{x_{0}^{\prime}}\right)$ in $T^{*}(\mathbb{R} \times \Gamma)$ is contained in $\cup_{j=1}^{N_{x_{0}^{\prime}}} \mathcal{O}_{j}^{ \pm}$.

(ii) $\mathcal{O}_{j}^{ \pm} \cap \mathcal{O}_{k}^{ \pm} \neq \emptyset$ for $|j-k| \geq 2,\left\{(t, \tau, \zeta) \in \mathcal{L}_{T}^{3, \pm}\left(U_{x_{0}^{\prime}}\right) ; t \geq \tau_{0}\right\} \subset \mathcal{O}_{0}^{ \pm}$and $\tilde{\mathcal{L}}_{T}^{3, \pm}\left(U_{x_{0}^{\prime}}\right) \subset \mathcal{O}_{N_{x_{0}^{\prime}}^{ \pm}}^{ \pm}$

(iii) There exists a local coordinate system $V_{j}$ of $\Gamma$ such that $\tilde{\pi}_{\Gamma}\left(\mathcal{O}_{j}^{ \pm}\right) \subset V_{j}$, where $\tilde{\pi}_{\Gamma}: T^{*}(\mathbb{R} \times \Gamma) \rightarrow \Gamma$ is the projection.

(iv) For $V_{j}$ in (iii), $T^{*}\left(V_{j}\right) \ni \zeta \mapsto\left(x^{(j)}(\zeta), p^{(j)}(\zeta)\right) \in \mathbb{R}^{n-1} \times \mathbb{R}^{n-1}$ denotes the local coordinate system of $T^{*}(\Gamma)$ induced by $V_{j}$. There exists a subset $K_{j}=$ $\left\{i_{1}, i_{2}, \cdots, i_{\left|K_{j}\right|}\right\} \subset\{1,2, \ldots, n-1\}$ such that

$$
\begin{aligned}
\mathcal{O}_{j}^{ \pm} \ni r^{ \pm}\left(s, y^{\prime}\right) \mapsto\left(s, x_{K_{j}^{\prime}}^{(j)}\left(h_{ \pm}^{s-\tau_{0}}\left(y^{\prime}, \pm c_{R}{ }^{-1} \theta\right)\right),\right. & \\
& \left.p_{K_{j}}^{(j)}\left(h_{ \pm}^{s-\tau_{0}}\left(y^{\prime}, \pm c_{R}{ }^{-1} \theta\right)\right)\right) \in \mathbb{R} \times \mathbb{R}^{n-1}
\end{aligned}
$$

is a local coordinate system, where $K_{j}^{\prime}=\{1,2, \ldots, n-1\} \backslash K_{j}(=$ $\left.\left\{j_{1}, j_{2}, \cdots, j_{\left|K_{j}^{\prime}\right|}\right\}\right)$ and $x_{K_{j}^{\prime}}^{(j)}(\zeta)=\left(x_{j_{1}}^{(j)}(\zeta), x_{j_{2}}^{(j)}(\zeta), \ldots, x_{j_{\left|K_{j}^{\prime}\right|}}^{(j)}(\zeta)\right), p_{K_{j}}^{(j)}(\zeta)=$ $\left(p_{i_{1}}^{(j)}(\zeta), p_{i_{2}}^{(j)}(\zeta), \ldots, p_{i_{\left|K_{j}\right|}}^{(j)}(\zeta)\right)$.

Let $g_{j}\left(x^{\prime}\right)(>0)$ be a $C^{\infty}$-function on $\mathbb{R} \times V_{j}$ defined by $d V_{\mathbb{R} \times \Gamma}=g_{j}^{\frac{1}{2}}\left(x^{\prime}\right)\left|d t \wedge d x^{(j)}\right|$. We denote by $d V_{ \pm}$the pull back of $d V_{\mathbb{R} \times \Gamma}$ by $C^{\infty}$ - immersion in $\mathcal{L}_{T}^{3, \pm}\left(\tilde{U}_{x_{0}^{\prime}}\right) \ni r\left(t, y^{\prime}\right) \mapsto$ 
$\left(t, y^{\prime}\right) \in \mathbb{R} \times \tilde{U}_{x_{0}^{\prime}}$. Let $J_{j}^{ \pm}(r)$ be a $C^{\infty}$-function on $\mathcal{O}_{j}^{ \pm}$given by $d V_{ \pm}=\left(J_{j}^{ \pm}(r)\right)^{-1} \mid d t \wedge$ $d x_{K_{j}^{\prime}}^{(j)} \wedge d p_{K_{j}}^{(j)} \mid$.

For each local coordinate system $\mathcal{O}_{j}^{ \pm}$given above, we define the precanonical operators $\mathcal{K}_{ \pm}\left(\mathcal{O}_{j}^{ \pm}, K_{j}\right): C^{\infty}\left(\mathcal{O}_{j}^{ \pm}\right) \rightarrow C_{0}^{\infty}(\mathbb{R} \times \Gamma)$ and $\tilde{\mathcal{K}}_{ \pm}\left(\mathcal{O}_{j}^{ \pm}, K_{j}\right): C^{\infty}\left(\mathcal{O}_{j}^{ \pm}\right) \rightarrow$ $C_{0}^{\infty}\left(\mathbb{R} \times \Gamma ; \Omega^{\frac{1}{2}}\right)$ by

$$
\begin{aligned}
&\left(\mathcal{K}_{ \pm}\left(\mathcal{O}_{j}^{ \pm}, K_{j}\right) \varphi\right)\left(t, x^{\prime}\right)=\chi_{j}\left(x^{\prime}\right) g_{j}^{\frac{1}{4}}\left(x^{\prime}\right) \\
& \mathcal{F}_{\tau ; p_{K_{j}}^{(j)} \rightarrow x_{K_{j}}^{(j)}}\left[\left.\frac{e^{i \tau\left(\tilde{S}_{ \pm}(r)-t\right)}}{J_{j}^{ \pm}(r)} \varphi(r)\right|_{r=r^{ \pm}\left(t, x_{K_{j}^{\prime}}^{(j)}, p_{K_{j}}^{(j)}\right)}\right]\left(t, x^{\prime}\right) \quad( \pm \tau>>1)
\end{aligned}
$$

and $\tilde{\mathcal{K}}_{ \pm}\left(\mathcal{O}_{j}^{ \pm}, K_{j}\right) \varphi=\mathcal{K}_{ \pm}\left(\mathcal{O}_{j}^{ \pm}, K_{j}\right) d V_{\mathbb{R} \times \Gamma}^{\frac{1}{2}}$, where $\chi_{j} \in C_{0}^{\infty}\left(V_{j}\right)$ with $\chi_{j}=1$ near $\tilde{\pi}\left(\mathcal{O}_{j}^{ \pm}\right), r^{ \pm}\left(t, x_{K_{j}^{\prime}}^{(j)}, p_{K_{j}}^{(j)}\right)$ is the inverse of the local coordinate system of $\mathcal{O}_{j}^{ \pm}$introduced in (iv), and

$$
\mathcal{F}_{\tau ; F_{K_{j}}^{(j)} \rightarrow x_{K_{j}}^{(j)}}\left[f\left(t, x_{K_{j}^{\prime}}, p_{K_{j}}\right)\right]=\left(\frac{|\tau|}{2 \pi}\right)^{\left|K_{j}\right| / 2} \int_{\mathbb{R}^{\left|K_{j}\right|}} e^{i \tau x_{K_{j}^{\prime}}^{(j)} \cdot p_{K_{j}}^{(j)}} f\left(t, x_{K_{j}^{\prime}}^{(j)}, p_{K_{j}}^{(j)}\right) d p_{K_{j}}^{(j)} .
$$

In (5.17), the phase function $\tilde{S}_{ \pm}(r)$ corresponding to the Lagrangian manifold $\mathcal{L}_{T}^{3, \pm}\left(\tilde{U}_{x_{0}^{\prime}}\right)$ is given by the integral of the canonical one-form on $\mathcal{L}_{T}^{3, \pm}\left(U_{x_{j}^{\prime}}\right): S_{j}^{ \pm}(r)=$ $\pm \int_{r_{0}}^{r} p d q+c_{R}^{-1} \theta \cdot y^{\prime}\left(r=r^{ \pm}\left(t, y^{\prime}\right)\right)$.

Using these $\tilde{\mathcal{K}}_{ \pm}\left(\mathcal{O}_{j}^{ \pm}, K_{j}\right)$, we define the Maslov canonical operator $K_{x_{0}^{\prime}}^{ \pm}$by

$$
K_{x_{0}^{\prime}}^{ \pm}[k]\left(t, x^{\prime}\right)=\sum_{j=0}^{N_{x_{0}^{\prime}}} e^{i \sigma\left(\mathcal{O}_{j}^{ \pm}\right)} \tilde{\mathcal{K}}_{ \pm}\left(\mathcal{O}_{j}^{ \pm}, K_{j}\right)\left[\tilde{\chi}_{j} k\right]\left(t, x^{\prime}\right) \quad\left(k \in C_{0}^{\infty}\left(\mathcal{L}_{T}^{3, \pm}\left(U_{x_{0}^{\prime}}\right)\right)\right)
$$

where $\tilde{\chi}_{j} \in C_{0}^{\infty}\left(\mathcal{O}_{j}^{ \pm}\right)$with $\sum_{j=0}^{N_{x_{0}^{\prime}}} \tilde{\chi}_{j}=1$ on $\mathcal{L}_{T}^{3, \pm}\left(U_{x_{0}^{\prime}}\right)$, and $\sigma\left(\mathcal{O}_{j}^{ \pm}\right)$are real constants given by the Maslov index.

The basic properties of the Maslov canonical operator $K_{x_{0}^{\prime}}^{ \pm}$are as follows:

Proposition 5.6. (i) For any $k \in C_{0}^{\infty}\left(\mathcal{L}_{T}^{3, \pm}\left(U_{x_{0}^{\prime}}\right)\right)$ and $[a, b] \subset I_{T}\left(s_{0}\right)(a, b \in \mathbb{R})$, we have

$$
\begin{aligned}
& \left\|K_{x_{0}^{\prime}}^{ \pm} k\right\|_{L^{2}\left([a, b] \times \Gamma ; \Omega^{\frac{1}{2}}\right)}^{2} \\
& =\int_{[a, b] \times U_{x_{0}^{\prime}}}\left|k\left(s, \mp 1, h_{ \pm}^{s-\tau_{0}}\left(x^{\prime}, \pm c_{R}^{-1} \theta\right)\right)\right|^{2} d s d x^{\prime}+O\left(\tau^{-1}\right) \quad \text { as } \tau \rightarrow \infty .
\end{aligned}
$$

(ii) For any $A \in \Psi_{\text {phg }}^{m}\left(\mathbb{R} \times \Gamma ; \Omega^{\frac{1}{2}}, \Omega^{\frac{1}{2}}\right)$, there exist pseudo-differential operators $\mathcal{W}_{l}^{ \pm}$ on $\mathcal{L}_{T}^{3, \pm}\left(U_{x_{0}^{\prime}}\right)$ of order less than $2 l$ such that for any $N \in \mathbb{N}$ and $m^{\prime} \geq 0$

$$
\begin{aligned}
& \left(A K_{x_{0}^{\prime}}^{ \pm} k\right)\left(t, x^{\prime}\right)=K_{x_{0}^{\prime}}^{ \pm}\left[\sum_{l=0}^{N-1}(i \tau)^{m-l} \mathcal{W}_{l}^{ \pm} k\right]\left(t, x^{\prime}\right)+\left(R_{N, \tau} k\right)\left(t, x^{\prime}\right), \\
& \left\|R_{N, \tau} k\right\|_{H^{m^{\prime}}\left(I_{T}\left(s_{0}\right) \times \Gamma ; \Omega^{\frac{1}{2}}\right)}=O\left(|\tau|^{m+m^{\prime}-N}\right) \quad\left(\text { for } k \in C_{0}^{\infty}\left(\mathcal{L}_{T}^{3, \pm}\left(U_{x_{0}^{\prime}}\right)\right)\right) .
\end{aligned}
$$


Note that $\mathcal{W}_{0}^{ \pm} k(r)=i^{-m} \sigma_{p}(A)(r) k(r)\left(\right.$ for $\left.r \in \mathcal{L}_{T}^{3, \pm}\left(U_{x_{0}^{\prime}}\right)\right)$.

(iii) If $A=A_{0} O p\left(\chi_{1} \lambda^{(e)}\right)_{\Omega^{\frac{1}{2}}}$ in (ii), we obtain

$$
\mathcal{W}_{1}^{ \pm} k(r)=i^{-1}\left\{\left(\frac{d}{d s}\right)_{ \pm}+i \sigma_{s p}\left(\lambda^{(e)}\right)(r)\right\} k(r) \quad\left(\text { for } r \in \mathcal{L}_{T}^{3, \pm}\left(U_{x_{0}^{\prime}}\right) \text { with } \chi_{1}(r)=1\right)
$$

where $\left(\frac{d}{d s}\right)_{ \pm}$denotes the differentiation along the phase flow $h_{ \pm}^{s}$, and $\sigma_{s p}\left(\lambda^{(e)}\right)(r)$ denotes the subprincipal symbol of the operator $O p\left(\chi_{1} \lambda^{(e)}\right)_{\Omega^{\frac{1}{2}}}$ on the set $\left\{r \in T^{*}(\mathbb{R} \times\right.$ $\left.\Gamma): \chi_{1}(r)=1\right\}$.

Using the stationary phase methods (cf. Matsumura [13]), we can obtain Proposition 5.6 by the same methods as in Ichinose [3, 4] or Maslov and Fedoriuk [12].

As shown later, $\tilde{k}_{\varphi}\left(t, x^{\prime} ; \theta\right)$ is made by summing up the approximate solutions $l_{x_{0}^{\prime}}$ of the equations

$$
\begin{aligned}
& A_{0} O p\left(\chi_{1} \lambda^{(e)}\right)_{\Omega^{\frac{1}{2}}} l_{x_{0}^{\prime}}\left(t, x^{\prime} ; \tau\right) \\
& \quad-e^{i \tau\left(c_{R}^{-1} \theta \cdot x^{\prime}-t\right)}\left(\tilde{\phi}_{5}\right)_{\theta}\left(t, x^{\prime}\right) a\left(t, x^{\prime}, \tau\right) \psi_{x_{0}^{\prime}}\left(x^{\prime}\right) d V_{\mathbb{R} \times \Gamma}^{\frac{1}{2}}=O\left(|\tau|^{-\infty}\right),
\end{aligned}
$$

where $\psi_{x_{0}^{\prime}}$ is a cutoff function in $C_{0}^{\infty}\left(U_{x_{0}^{\prime}}\right)$ that is determined later. We can make this approximate solution $l_{x_{0}^{\prime}}\left(t, x^{\prime} ; \tau\right)$ by constructing its asymptotic expansion $l_{x_{0}^{\prime}}\left(t, x^{\prime} ; \tau\right) \sim \sum_{j=1}^{\infty} K_{x_{0}^{\prime}}^{ \pm}\left[\phi_{6}\left(\pi_{\mathbb{R}}(r)\right) \tilde{l}_{x_{0}^{\prime}, j}(r, \tau)(i|\tau|)^{1-j}\right]$. Here, $\pi_{\mathbb{R}}$ is the mapping: $r=(t, \tau, \zeta) \mapsto t, \phi_{6}(t)$ is a cutoff function in $C_{0}^{\infty}(\mathbb{R})$ that satisfies $0 \leq \phi_{6} \leq 1$, $\phi_{6}(t)=1$ if $\left|t+s_{0}\right| \leq t_{1}+5 c_{R}^{-1}$ and $\phi_{6}(t)=0$ if $\left|t+s_{0}\right| \geq t_{1}+6 c_{R}^{-1}$, and $\tilde{l}_{x_{0}^{\prime}, j}$ $(j=0,1, \ldots)$ are determined inductively as follows. Let $\tilde{a}_{1-j}(r, \tau)$ be the lift of $\left(\tilde{\phi}_{5}\right)_{\theta}\left(t, x^{\prime}\right) a_{1-j}\left(t, x^{\prime}, \tau\right) \psi_{x_{0}^{\prime}}\left(x^{\prime}\right)$ to the Lagrangian manifold $\mathcal{L}_{T}^{3, \pm}\left(U_{x_{0}^{\prime}}\right)$. Noting that $\operatorname{supp}\left[\psi_{x_{0}^{\prime}}\right] \subset U_{x_{0}^{\prime}} \subset \partial \mathbb{R}_{+}^{3} \backslash B_{r_{0}}, \mathcal{O}_{0}^{ \pm} \subset T^{*}\left(\mathbb{R} \times \partial \mathbb{R}_{+}^{3}\right)$ and chooseing $K_{0}=\emptyset$, by Proposition 5.6 we have the following equations of $\tilde{l}_{x_{0}^{\prime}, j}(r, \tau)(j=0,1, \ldots)$ :

$$
\begin{aligned}
i^{-1}\left\{\left(\frac{d}{d s}\right)_{ \pm}+i \sigma_{s p}\left(\lambda^{(e)}\right)(r)\right\} \tilde{l}_{x_{0}^{\prime}, 0}(r, \tau) & =i^{-1} \tilde{a}_{1}(r, \tau)|\tau|^{-1} \\
i^{-1}\left\{\left(\frac{d}{d s}\right)_{ \pm}+i \sigma_{s p}\left(\lambda^{(e)}\right)(r)\right\} \tilde{l}_{x_{0}^{\prime}, j}(r, \tau) & =i^{j-1} \tilde{a}_{1-j}(r, \tau)|\tau|^{j-1} \\
& -\sum_{p=0}^{j-1} \mathcal{W}_{j+1-p}^{ \pm} \tilde{l}_{x_{0}^{\prime}, p}(r, \tau) \quad(j=1,2, \ldots) .
\end{aligned}
$$

Using these solutions $\tilde{l}_{x_{0}^{\prime}, j}(r, \tau)$, we set the function

$$
l_{x_{0}^{\prime}}^{(N)}\left(t, x^{\prime} ; \tau\right)=\sum_{j=0}^{N} K_{x_{0}^{\prime}}^{ \pm}\left[\phi_{6}\left(\pi_{\mathbb{R}}(r)\right) \tilde{l}_{x_{0}^{\prime}, j}(r, \tau)(i|\tau|)^{1-j}\right] \quad( \pm \tau>1)
$$

for any non-negative integer $N$. Then, it follows that

$$
\begin{gathered}
\left\|O p\left(\chi_{1} \lambda^{(e)}\right)_{\Omega^{\frac{1}{2}}} l_{x_{0}^{\prime}}^{(N)}-e^{i \tau\left(c_{R}^{-1} \theta \cdot x^{\prime}-t\right)}\left(\tilde{\phi}_{5}\right)_{\theta}\left(t, x^{\prime}\right) a\left(t, x^{\prime}, \tau\right) \psi_{x_{0}^{\prime}}\left(x^{\prime}\right) d V_{\mathbb{R}^{\frac{1}{2}} \times \Gamma}\right\|_{H^{m}\left(I_{t_{1}+5 c_{R}^{-1}(s) \times \Gamma ; \Omega^{\frac{1}{2}}}\right)} \\
=O\left(|\tau|^{m-N}\right) \quad \text { as }|\tau| \rightarrow \infty .
\end{gathered}
$$


Thus, choosing $\tilde{l}_{x_{0}^{\prime}}(r, \tau) \sim \sum_{j=0}^{\infty} \tilde{l}_{x_{0}^{\prime}, j}(r, \tau)(i|\tau|)^{1-j}$, we obtain the approximate solution $l_{x_{0}^{\prime}}\left(t, x^{\prime} ; \tau\right)$ of $(5.18)$ by

$$
l_{x_{0}^{\prime}}\left(t, x^{\prime} ; \tau\right)=K_{x_{0}^{\prime}}^{ \pm}\left[\phi_{6}\left(\pi_{\mathbb{R}}(r)\right) \tilde{l}_{x_{0}^{\prime}}(r, \tau)\right]\left(t, x^{\prime}\right) \quad( \pm \tau>1) .
$$

Since $L_{r_{0}}^{+} \cup L_{r_{0}}^{-} \cup L_{r_{0}}^{0}$ is a compact set, we can take a finite number of points $x_{1}^{\prime}, \ldots, x_{N_{0}}^{\prime} \in L_{r_{0}}^{+}$and $x_{N_{0}+1}^{\prime}, \ldots, x_{N_{1}}^{\prime} \in L_{r_{0}}^{-} \cup L_{r_{0}}^{0}$ such that the open sets $U_{x_{j}^{\prime}} \ni x_{j}^{\prime}$ $\left(j=1,2, \ldots, N_{1}\right)$ consist of a finite open covering of $L_{r_{0}}^{+} \cup L_{r_{0}}^{-} \cup L_{r_{0}}^{0}$ in each of which the construction of the approximate solution of (5.18) is valid. We choose a partition $\left\{\psi_{x_{j}^{\prime}}\right\}_{j=1, \ldots, N_{1}}$ of unity with $\operatorname{supp}\left[\psi_{x_{j}^{\prime}}\right] \subset U_{x_{j}^{\prime}}$ and $\sum_{j=1}^{N_{1}} \psi_{x_{j}^{\prime}} \tilde{\psi}=\tilde{\psi}$, and construct the same approximate solutions $l_{x_{j}^{\prime}}\left(t, x^{\prime}, \tau\right)\left(j=1,2, \ldots, N_{1}\right)$ in each $U_{x_{j}^{\prime}}$ (replacing $\psi_{x_{0}}^{\prime}$ with $\psi_{x_{j}}^{\prime}$ ) as shown in (5.18). Then, we see that the function

$$
\tilde{k}_{\varphi}\left(t, x^{\prime} ; \theta\right)=\frac{1}{2 \pi} \int_{\mathbb{R}} \sum_{j=1}^{N_{1}} l_{x_{j}^{\prime}}\left(t, x^{\prime}, \tau\right) \phi_{7}(\tau) \hat{\varphi}(\tau) d \tau
$$

is the required solution for (5.10), where $\phi_{7}(\tau)$ is a $C^{\infty}$ function satisfying $0 \leq \phi_{7} \leq 1$, $\phi_{7}(\tau)=1$ if $|\tau| \geq 2$ and $\phi_{7}(\tau)=0$ if $|\tau| \leq 1$. Thus, we finish the construction of all the approximate solutions introduced in sections 2 and 3.

At the end of this section, we explain the properties of $\tilde{k}_{\varphi}\left(t, x^{\prime} ; \theta\right)$ that are needed later for proof of the main theorems. We see from the construction of $\tilde{k}_{\varphi}$ that

$$
\operatorname{supp}\left[\tilde{k}_{\varphi}\right] \subset\left[-\left(s_{0}+t_{1}+6 c_{R}^{-1}\right),-s_{0}+t_{1}+6 c_{R}^{-1}\right] \times\left(B_{r_{3}} \cap \Gamma\right)
$$

for a constant $r_{3}>0$ depending only on $t_{1}>0$. The mapping: $\left(s, y^{\prime}\right) \mapsto\left(s, h_{ \pm}^{s-\tau_{0}}\left(y^{\prime}\right.\right.$, $\left.\left.\pm c_{R}^{-1} \theta\right)\right)$ is diffeomorphic for $s \leq-s_{0}-t_{1}$ and $y^{\prime} \in U_{x_{j}^{\prime}}\left(j=1,2, \ldots, N_{1}\right)$. If $\left.h_{+}^{\tilde{s}}\left(y^{\prime}, c_{R}^{-1} \theta\right) \in T^{*}(\Gamma)\right|_{\left(B_{r_{0}}\right)^{c}}$, we can write $h_{+}^{\tilde{s}}$ in the form $h_{+}^{\tilde{s}}\left(y^{\prime}, c_{R}^{-1} \theta\right)=\left(q\left(\tilde{s}, y^{\prime}, c_{R}^{-1} \theta\right)\right.$, $\left.p\left(\tilde{s}, y^{\prime}, c_{R}^{-1} \theta\right)\right)$. Set $X\left(s, y^{\prime}\right)=\pi_{\Gamma}\left(h_{+}^{s-\tau_{0}}\left(y^{\prime}, c_{R}^{-1} \theta\right)\right)$, and $\Theta\left(y^{\prime}\right)=c_{R} p\left(-s_{0}-t_{1}-\right.$ $\left.\tau_{0}, y, c_{R}^{-1} \theta\right)$. Then, using (5.13), we have $X\left(s, y^{\prime}\right)=\pi_{\Gamma}\left(h_{-}^{s-\tau_{0}}\left(y^{\prime},-c_{R}^{-1} \theta\right)\right)$; using (5.16), $X\left(t, y^{\prime}\right)=X\left(-s_{0}-t_{1}, y^{\prime}\right)+c_{R}\left(t+s_{0}+t_{1}\right) \Theta\left(y^{\prime}\right)\left(\right.$ for $\left.t \leq-s_{0}-t_{1}\right)$.

From (5.16), we can take $K_{N_{x_{0}^{\prime}}}=\emptyset$. Hence, each $l_{x_{j}^{\prime}}\left(t, x^{\prime}, \tau\right)$ in (5.22) satisfies

$$
\begin{aligned}
l_{x_{j}^{\prime}}\left(t, \tilde{X}\left(t, y^{\prime}\right), \tau\right) & =K_{x_{j}^{\prime}}^{ \pm}\left[\phi_{6}\left(\pi_{\mathbb{R}}(r)\right) \tilde{l}_{x_{j}^{\prime}}(r, \tau)\right]\left(t, \tilde{X}\left(t, y^{\prime}\right)\right) \\
& =\frac{e^{-\frac{\pi}{2} i m_{j}}}{\left|\operatorname{det}\left(\frac{\partial X}{\partial y^{\prime}}\right)\left(t, y^{\prime}\right)\right|^{1 / 2}} e^{i \tau\left(S_{j}^{ \pm}\left(t, y^{\prime}\right)-t\right)} \phi_{6}(t) \tilde{l}_{x_{j}^{\prime}}\left(r\left(t, y^{\prime}\right), \tau\right) \quad( \pm \tau>0),
\end{aligned}
$$

where $S_{j}^{ \pm}\left(t, y^{\prime}\right)=\tilde{S}_{j}^{ \pm}\left(r^{ \pm}\left(t, y^{\prime}\right)\right)$, and $m_{j}$ is the Morse index of the path $\gamma_{+}^{(j)}\left(t, y^{\prime}\right)$ : $\left[-s_{0}-t_{1}, \tau_{0}\right] \ni s \mapsto r^{+}\left(s, y^{\prime}\right) \in \mathcal{L}_{T}^{3,+}\left(U_{x_{j}^{\prime}}\right)$. Note that $m_{j}$ coincides with the Morse index of the path $\gamma_{-}^{(j)}\left(t, y^{\prime}\right):\left[-s_{0}-t_{1}, \tau_{0}\right] \ni s \mapsto r^{-}\left(s, y^{\prime}\right) \in \mathcal{L}_{T}^{3,-}\left(U_{x_{j}^{\prime}}\right)$ since (5.13) yields $\check{I}_{\mathbb{R} \times \Gamma}\left(\mathcal{L}_{T}^{3,-}\left(U_{x_{j}^{\prime}}\right)\right)=\mathcal{L}_{T}^{3,+}\left(U_{x_{j}^{\prime}}\right)$.

From (5.13), we see that $S_{j}^{+}\left(t, y^{\prime}\right)=S_{j}^{-}\left(t, y^{\prime}\right)$. We write $S_{j}\left(t, y^{\prime}\right)=S_{j}^{+}\left(t, y^{\prime}\right)=$ $S_{j}^{-}\left(t, y^{\prime}\right) . S_{j}\left(t, y^{\prime}\right)$ is expressed of the form

$$
S_{j}\left(t, y^{\prime}\right)=\int_{\gamma_{+}^{(j)}\left(t, y^{\prime}\right)} p d q+c_{R}^{-1} \theta \cdot y^{\prime}
$$


Note that it follows from (5.19), (5.20) and (5.15) that for any $i=0,1, \ldots$

$$
\begin{array}{r}
\operatorname{supp}\left[l_{x_{j}^{\prime}, i}\left(\pi^{-1}(\cdot, \cdot), \tau\right)\right] \subset\left\{\left(t, \pi_{\Gamma}\left(h_{ \pm}^{t-\tau_{0}}\left(y^{\prime}, \pm c_{R}^{-1} \theta\right)\right)\right) \in \mathbb{R} \times \Gamma\right. \\
t \leq \tau_{0}, y^{\prime} \in U_{x_{j}^{\prime}}, y^{\prime}+c_{R}\left(s-\tau_{0}\right) \theta \in \operatorname{supp}\left[\psi_{x_{j}^{\prime}}\right]\left(\text { for some } s \geq \tau_{0}-2 c_{R}^{-1}\right), \\
\left.\left|c_{R}^{-1} \theta \cdot y^{\prime}-\left(\tau_{0}+s_{0}\right)\right| \leq 2 \epsilon\right\}
\end{array}
$$

For $\omega, \theta \in S^{1}$ and $y^{\prime} \in \Gamma \cap\left(B_{r_{0}}\right)^{c}$, we put

$$
s_{\theta}^{-}\left(\omega ; y^{\prime}\right)=\lim _{t \rightarrow-\infty}\left(c_{R}^{-1} q\left(t-c_{R}^{-1} \theta \cdot y^{\prime}, y^{\prime}, c_{R}^{-1} \theta\right)-t c_{R} p\left(t-c_{R}^{-1} \theta \cdot y^{\prime}, y^{\prime}, c_{R}^{-1} \theta\right)\right) \cdot \omega .
$$

Then, using (5.14) and the equality $H\left(y^{\prime}, \xi\right)=c_{R}|\xi|$ for $\left.\left(y^{\prime}, \xi\right) \in T^{*}(\Gamma)\right|_{\left(B_{r_{0}}\right)^{c}}$, we have

$$
s_{\theta}^{-}\left(\omega ; y^{\prime}\right)=c_{R}^{-1} \omega \cdot \pi_{\Gamma}\left(h_{ \pm}^{t-\tau_{0}}\left(y^{\prime}, \pm c_{R}^{-1} \theta\right)\right)-\left(t+c_{R}^{-1} \theta \cdot y^{\prime}-\tau_{0}\right) \omega \cdot \Theta\left(y^{\prime}\right)
$$

for $t \leq-s_{0}-t_{1}$. Furthermore, using $c_{R}^{-1} \theta \cdot x_{j}^{\prime}=s_{0}+\tau_{0}$, we obtain

$$
\begin{aligned}
c_{R}^{-1} \omega \cdot \pi_{\Gamma}\left(h_{ \pm}^{t-\tau_{0}}\left(y^{\prime}, \pm c_{R}^{-1} \theta\right)\right)=s_{0} & +s_{\theta}^{-}\left(\omega ; y^{\prime}\right)+t+c_{R}^{-1} \theta \cdot\left(y^{\prime}-x_{j}^{\prime}\right) \\
& +\left(\omega \cdot \Theta\left(y^{\prime}\right)-1\right)\left(t-\tau_{0}+c_{R}^{-1} \theta \cdot y^{\prime}\right) .
\end{aligned}
$$

Since it follows from (5.27) that $c_{R}^{-1} \omega \cdot \pi_{\Gamma}\left(h_{ \pm}^{t-\tau_{0}}\left(y^{\prime}, \pm c_{R}^{-1} \theta\right)\right) \geq s_{0}+s_{\theta}^{-}\left(\omega ; y^{\prime}\right)+t-2 \epsilon$ for $t \leq-s_{0}-t_{1}$, and from the defnitions of $s_{\theta}^{-}\left(\omega ; y^{\prime}\right)$ and $s_{\omega}^{+}(\theta)$ that $s_{\theta}^{-}\left(\omega ; y^{\prime}\right) \geq-s_{\omega}^{+}(\theta)$, we have by $(5.25)$

$$
\left\{\omega \cdot x^{\prime} ; x^{\prime} \in \operatorname{supp}\left[l_{x_{j}^{\prime}}(t, \cdot, \tau)\right]\right\} \subset\left[c_{R} t+c_{R}\left(s_{0}-s_{\omega}^{+}(\theta)-2 \epsilon\right), \infty\right)
$$

(for $\tau \in \mathbb{R}, t \leq-s_{0}-t_{1}$ ).

6. Singularities of $S_{\mathrm{RR}}(s, \theta, \omega)$. In this section we study singularities of $S_{\mathrm{RR}}(s, \theta, \omega)$. According to Theorem 3.1 , we have only to examine $J_{0}(\varphi)$. We have $\left.\left(\partial_{t}-c_{R} \partial_{\omega}\right) v_{\varphi}^{R}(t, x ; \theta)\right|_{x_{3}=0}=\left.\left(\partial_{t}-c_{R} \partial_{\omega}\right) v_{\varphi}^{R, b}(t, x ; \theta)\right|_{x_{3}=0}$ since $\left.v_{\varphi}^{R}(t, x ; \theta)\right|_{x_{3}=0}=$ $\left.v_{\varphi}^{R, b}(t, x ; \theta)\right|_{x_{3}=0}$ (cf. (4.4), (4.3)) and the derivative $\partial_{\omega}=\sum_{i=1}^{2} \omega_{i} \partial_{x_{i}}$ is tangent to $\operatorname{supp}\left[\psi_{2}\right] \cap \Gamma$. This fact, (4.21) and Theorem 3.1 imply that

$$
\begin{aligned}
& J_{0}(\varphi) \simeq-\int_{\Gamma} \phi_{2}(s) \partial_{s} \phi_{1}(s) \tilde{\psi}\left(x^{\prime}\right) \\
& \left.\quad{ }^{t} G_{1}\left[\phi_{0}(s) \psi_{1}\left(x^{\prime}\right)\left(\partial_{t}-c_{R} \partial_{\omega}\right) v_{\varphi}^{R, b}\left(t, x^{\prime} ; \theta\right)\right]\right|_{t=c_{R}^{-1} \omega \cdot x^{\prime}} d S_{x^{\prime}}
\end{aligned}
$$

with respect to $C_{0}^{\infty}(I) \rightarrow \mathbb{C}$.

Noting that $t_{1}>-s_{0}+c_{R}^{-1}\left(r_{0}+5\right)$ and (4.23), we have

$$
\begin{gathered}
\text { ess.supp }{ }^{t} G_{1} \cap\left\{\left(t, \tau, x^{\prime}, \xi\right) \in T^{*}\left(\mathbb{R} \times \partial \mathbb{R}_{+}^{3}\right) ; t=c_{R}^{-1} \omega \cdot x^{\prime},\left|x^{\prime}\right| \geq r_{0}+1\right\} \\
\subset\left\{\left(t, \tau, x^{\prime}, \xi\right) ; \omega \cdot x^{\prime} \leq-\left(r_{0}+6\right)\right\} .
\end{gathered}
$$

Proposition 4.5 and (6.2) yield

$$
\begin{gathered}
\int_{\Gamma} \phi_{2}(s) \partial_{s} \phi_{1}(s) \tilde{\psi}\left(x^{\prime}\right)^{t} G_{1}\left[\phi_{0}(s) \psi_{1}\left(x^{\prime}\right)\left(\partial_{t}-c_{R} \partial_{\omega}\right)\right. \\
\left.O p\left(\chi_{1}\right) \tilde{Q} O p\left(\chi_{2}\right) \psi_{2} F_{1} \tilde{\varphi}_{\theta}\right]\left.\right|_{t=c_{R}^{-1} \omega \cdot x^{\prime}}\left(t, x^{\prime}\right) d S_{x^{\prime}} \simeq 0 .
\end{gathered}
$$


Hence, using (5.9), (5.8) and (6.1), we obatin

$$
\begin{aligned}
J_{0}(\varphi) \simeq-\int_{\Gamma} \phi_{2}(s) \partial_{s} \phi_{1}(s) \tilde{\psi}\left(x^{\prime}\right)^{t} G_{1}\left[\phi_{0}(s) \psi_{1}\left(x^{\prime}\right)\left(\partial_{t}-c_{R} \partial_{\omega}\right)\right. \\
\left.O p\left(\chi_{1}\right) \tilde{E}^{(0)} k_{\varphi}\right]\left(c_{R}^{-1} \omega \cdot x^{\prime}, x^{\prime}\right) d S_{x^{\prime}} .
\end{aligned}
$$

Put $D=-\phi_{2}(s) \partial_{s} \phi_{1}(s) \tilde{\psi}\left(x^{\prime}\right)\left({ }^{t} G_{1} \phi_{0}(s) \psi_{1}\left(x^{\prime}\right)\left(\partial_{t}-c_{R} \partial_{\omega}\right) O p\left(\chi_{1}\right) \tilde{E}^{(0)}\right)_{\Omega^{\frac{1}{2}}} \in$ $\Psi_{p h g}^{0}\left(\mathbb{R} \times \Gamma ; \Omega^{\frac{1}{2}}, \Omega^{\frac{1}{2}}\right)$, and define the map $I_{\Omega^{-\frac{1}{2}}}: C_{0}^{\infty}\left(\mathbb{R} \times \Gamma ; \Omega^{\frac{1}{2}}\right) \mapsto C_{0}^{\infty}(\mathbb{R} \times \Gamma ; \mathbb{C})$ by $\left(I_{\Omega^{-\frac{1}{2}}} f\right) d V_{\mathbb{R} \times \Omega^{\frac{1}{2}}}=f$. Since $\phi_{0}(s)=1$ in a neighborhood of supp $\left[\phi_{2} \partial_{t} \phi_{1}\right]$, and $\psi_{1}\left(x^{\prime}\right)=1$ in a neighborhood of supp $[\tilde{\psi}]$, using (4.23) and (5.12), we can regard (6.3) as

$$
\left.J_{0}(\varphi) \simeq \int_{\Gamma}\left(I_{\Omega^{-\frac{1}{2}}} D\left(\phi_{0} \psi_{1} \tilde{k}_{\varphi}(\cdot, \cdot ; \theta)\right)\right)\right|_{t=c_{R}^{-1} \omega \cdot x^{\prime}} d S_{x^{\prime}} .
$$

From (4.22) and Lemma 5.3, the principal symbol $\sigma_{p}(D)$ of $D$ is of the form

$$
\begin{aligned}
\sigma_{p}(D)\left(t, \tau, x^{\prime}, \xi\right) & =i \alpha^{(0)} \chi_{1}\left(t, \tau, x^{\prime}, \xi\right) \phi_{0}(t) \psi_{1}\left(x^{\prime}\right)\left(\tau-c_{R} \omega \cdot \xi\right)\left|e_{0}^{0,(0)}(\tau, \xi)\right|^{-1} \\
& \int_{0}^{\infty}{ }^{t}\left(V_{-}^{(0)}\left(y_{3} ;-\tau,-\xi\right) e_{0}^{0,(0)}(-\tau,-\xi)\right) \overline{V_{-}^{(0)}\left(y_{3} ;-\tau,-\xi\right)} e_{0}^{0,(0)}(\tau, \xi) d y_{3} .
\end{aligned}
$$

From (iv) of Proposition 4.1, it follows that $e_{0}^{0,(0)}(-\tau,-\xi)=\overline{e_{0}^{0,(0)}(\tau, \xi)}$. Noting that the constant $C_{0}^{R}$ in $\phi_{0}^{R}$ is chosen so that $\left\|\phi_{0}^{(R)}\left(x^{\prime}, \cdot ; \tau, \tilde{\theta}\right)\right\|_{L^{2}([0, \infty))}^{2}=2 \pi \rho_{0} c_{R}|\tau|^{-1}$ (cf. (2.2)), and using (4.8) and the equality $2 c_{R} \alpha^{(0)}\left|e_{0}^{0,(0)}\left(-\tau,-\tau c_{R}^{-1} \tilde{\theta}\right)\right|=\alpha^{(1)}$ (for any $\tau \in \mathbb{R}$ and $\tilde{\theta} \in S^{1}$ ), we obtain

$$
\begin{array}{r}
\left.\sigma_{p}(D)\left(t, \tau, x^{\prime}, \xi\right)\right|_{\xi=-\tau c_{R}^{-1} \tilde{\theta}}=4 \pi i \rho_{0} c_{R}^{2}\left(\alpha^{(1)}\right)^{-1} \phi_{0}(t) \psi_{1}\left(x^{\prime}\right)|\tau|^{-1} \tau \\
(1+\omega \cdot \tilde{\theta}) \chi_{1}\left(t, \tau, x^{\prime},-\tau c_{R}^{-1} \tilde{\theta}\right) .
\end{array}
$$

(5.22), (5.21) and Proposition 5.6 yield

$$
\begin{aligned}
J_{0}(\varphi) \simeq & \sum_{j=1}^{N_{1}} \int_{\partial \mathbb{R}_{+}^{3}} \phi_{2}(t) \partial_{t} \phi_{1}(t) \tilde{\psi}\left(x^{\prime}\right) \\
& \left\{\left.\int_{0}^{\infty} I_{\Omega^{-\frac{1}{2}}}\left\{K_{x_{j}^{\prime}}^{+}\left[\sum_{q=0}^{N} b_{j, q}(r, 1)(i|\tau|)^{1-q}\right]\left(t, x^{\prime}\right)\right\}\right|_{t=c_{R}^{-1} \omega \cdot x^{\prime}} \phi_{7}(\tau) \hat{\varphi}(\tau) d \tau \quad(6.5)\right. \\
& \left.+\left.\int_{-\infty}^{0} I_{\Omega^{-\frac{1}{2}}}\left\{K_{x_{j}^{\prime}}^{-}\left[\sum_{q=0}^{N} b_{j, q}(r,-1)(i|\tau|)^{1-q}\right]\left(t, x^{\prime}\right)\right\}\right|_{t=c_{R}^{-1} \omega \cdot x^{\prime}} \phi_{7}(\tau) \hat{\varphi}(\tau) d \tau\right\} d x^{\prime} \\
& +R_{N}(\varphi) .
\end{aligned}
$$

In (6.5), each $b_{j, q}(r, \tau) \in C_{0}^{\infty}\left(\mathcal{L}_{T}^{3, \pm}\left(U_{x_{0}^{\prime}}\right)\right)(q=0,1,2, \ldots)$ is homogeneous of order 0 with respect to $\tau(|\tau| \geq 1)$, and $b_{j, 0}$ is of the form

$$
b_{j, 0}(r, \tau)=(2 \pi)^{-1} \phi_{6}\left(\pi_{\mathbb{R}}(r)\right) \sigma_{p}(D)(r) l_{x_{j}^{\prime}, 0}(r, \tau) .
$$

The remainder terms $R_{N}(\varphi)$ in (6.5) are given by

$$
R_{N}(\varphi)=\int_{\partial \mathbb{R}_{+}^{3}} \int_{\mathbb{R}} \sum_{j=1}^{N_{1}} R_{N, \tau, j}\left(c_{R}^{-1} \omega \cdot x^{\prime}, x^{\prime}\right) \phi_{7}(\tau) \hat{\varphi}(\tau) d \tau d x^{\prime},
$$


where $R_{N, \tau, j} \in C_{0}^{\infty}(\mathbb{R} \times \Gamma ; \mathbb{C})$ satisfies $\operatorname{supp}\left[R_{N, \tau, j}\right] \subset \operatorname{supp}\left[\phi_{0}\right] \times\left(\operatorname{supp}\left[\psi_{1}\right] \cap B_{r_{1}}\right)$ and

$\left\|R_{N, \tau, j}\right\|_{H^{2}\left(I_{t_{1}+6 c_{R}^{-1}\left(s_{0}\right)} \times \Gamma\right)} \leq C_{N, j}(1+|\tau|)^{2-N} \quad$ (for any $\tau \in \mathbb{R}$ and $\left.j, N=1,2, \ldots\right)$.

Hence, we have $\left|R_{N}(\varphi)\right| \leq C_{N}\|\varphi\|_{H^{2-N}(\mathbb{R})} \quad\left(\right.$ for any $\varphi \in C_{0}^{\infty}\left(\left(s_{0}-\epsilon, s_{0}+\epsilon\right)\right)$ ), which means that the distributions $R_{N}$ belong to $H^{N-2}\left(s_{0}-\epsilon, s_{0}+\epsilon\right)$.

From (4.23), it follows that $\pi\left(\operatorname{supp}\left[b_{j, q}\right]\right) \subset\left[-s_{0}-\left(t_{1}+3 c_{R}^{-1}\right),-s_{0}-\left(t_{1}+c_{R}^{-1}\right)\right] \times$ $\left(B_{r_{0}+1}\right)^{c}$ since ess.supp $D \subset(\operatorname{supp}[\phi] \times \operatorname{supp}[\tilde{\psi}]) \times \mathbb{R}_{(\tau, \xi)}^{3}$. This fact, (5.23) and (6.5) imply that

$$
J_{0}(\varphi) \simeq \sum_{j=1}^{N_{1}} \sum_{q=0}^{N-1} I_{j, q}(\varphi)+R_{N}(\varphi) \quad \text { with respect to } C_{0}^{\infty}(I) \rightarrow \mathbb{C},
$$

where

$$
\begin{array}{r}
I_{j, q}(\varphi)=\left.\int_{\mathbb{R}} \int_{\partial \mathbb{R}_{+}^{3}} \phi_{2}(t) \partial_{t} \phi_{1}(t) \tilde{\psi}\left(x^{\prime}\right)\left\{e^{i \tau\left(\tilde{S}_{j}\left(t, x^{\prime}\right)-t\right)} \tilde{b}_{j, q}\left(t, x^{\prime}, \tau\right)\right\}\right|_{t=c_{R}^{-1} \omega \cdot x^{\prime}} \\
(i \tau)^{1-q} \phi_{7}(\tau) \hat{\varphi}(\tau) d \tau d x^{\prime} .
\end{array}
$$

Here, $\tilde{b}_{j, q}\left(t, x^{\prime}, \tau\right)$ are defined by

$$
\begin{aligned}
& \tilde{b}_{j, q}\left(t, X\left(t, y^{\prime}\right), \tau\right) \\
& =e^{-i m_{j} \pi / 2} b_{j, q}\left(r^{ \pm}\left(t, y^{\prime}\right), \tau\right)\left(\tau|\tau|^{-1}\right)^{q-1}\left|\operatorname{det}\left(\partial_{y^{\prime}} X\left(t, y^{\prime}\right)\right)\right|^{-1 / 2} \quad( \pm \tau>0),
\end{aligned}
$$

and $\tilde{S}_{j}\left(t, x^{\prime}\right)$ are defined by $\tilde{S}_{j}\left(t, X\left(t, y^{\prime}\right)\right)=S_{j}\left(t, y^{\prime}\right)$ for $t \in \mathbb{R}$ and $y^{\prime} \in U_{x_{j}^{\prime}}$ (where $S_{j}\left(t, y^{\prime}\right)$ are defined in (5.24)).

Proof of Theorem 1.1. Let $s_{0}>s_{\omega}^{+}(\theta)$. We choose $\epsilon>0$ with $\epsilon^{\prime}=s_{0}-s_{\omega}^{+}(\theta)-3 \epsilon>$ 0 . From (5.28), we have $\left\{c_{R}^{-1} \omega \cdot x^{\prime} ;(t, x) \in \operatorname{supp}\left[\tilde{b}_{j, p}\right]\right\} \subset\left(t+\epsilon^{\prime}, \infty\right)$ for any $j$ and $q$; this means that $\tilde{b}_{j, p}\left(c_{R}^{-1} \omega \cdot x^{\prime}, x^{\prime}\right)=0$. Thus, using $(6.7)$ and $(6.8)$, we have $J_{0}(\varphi) \simeq$ $R_{N}(\varphi)$. From this fact and Theorem 3.1, we obtain $\left.s_{0} \notin \operatorname{sing} . \operatorname{supp} \tilde{S} S_{\mathrm{RR}}(\cdot, \theta, \omega)\right]$, which completes the proof of Theorem 1.1.

To show Theorem 1.2, we need more precise forms of the oscillatory integrals in (6.8). We put $K_{j}\left(t, y^{\prime}, \omega, \theta\right)=s_{0}+s_{\theta}^{-}\left(\omega ; y^{\prime}\right)+\left(t-\tau_{0}\right)\left(\omega \cdot \Theta\left(y^{\prime}\right)-1\right)+c_{R}^{-1} \theta \cdot(\omega$. $\left.\Theta\left(y^{\prime}\right) y^{\prime}-x_{j}^{\prime}\right)$. From (5.27), $K_{j}\left(t^{\prime}, y^{\prime}, \omega, \theta\right)=0$ is equivalent to $c_{R}^{-1} \omega \cdot X\left(t^{\prime}, y^{\prime}\right)-t^{\prime}=0$. This fact and (5.25) imply that

$$
\begin{gathered}
\operatorname{supp}_{x^{\prime}}\left[\cup_{q=0}^{\infty} \tilde{b}_{j, q}\left(c_{R}^{-1} \omega \cdot x^{\prime}, x^{\prime}, \tau\right)\right] \subset\left\{x^{\prime} \in \partial \mathbb{R}_{+}^{3} ; x^{\prime}=X\left(t^{\prime}, y^{\prime}\right) \text { for }\left(t^{\prime}, y^{\prime}\right)\right. \\
\left.\quad \in \mathbb{R} \times \operatorname{supp}\left[\psi_{x_{j}^{\prime}}\right],\left|c_{R}^{-1} \theta \cdot\left(y^{\prime}-x_{j}^{\prime}\right)\right| \leq 2 \epsilon, K_{j}\left(t^{\prime}, y^{\prime}, \omega, \theta\right)=0\right\} .
\end{gathered}
$$

Hereafter, we fix $s_{0}$ as $s_{0}=s_{\omega}^{+}(\theta)$. We define $M_{\theta}^{-}(\omega)$ and $s_{\omega}^{-}(\theta)$ by

$$
\begin{aligned}
M_{\theta}^{-}(\omega) & =\left\{y^{\prime} \in \Gamma ; \theta \cdot y^{\prime}=r_{0}, \lim _{t \rightarrow-\infty} p\left(t-c_{R}^{-1} r_{0}, y^{\prime}, c_{R}^{-1} \theta\right)=c_{R}^{-1} \omega\right\} \quad \text { and } \\
s_{\theta}^{-}(\omega) & =\inf _{y^{\prime} \in M_{\theta}^{-}(\omega)} \lim _{t \rightarrow-\infty}\left(c_{R}^{-1} q\left(t-c_{R}^{-1} r_{0}, y^{\prime} ; \theta\right) \cdot \omega-t\right)\left(=\inf _{y^{\prime} \in M_{\omega}^{-}(\theta)} s_{\theta}^{-}\left(\omega ; y^{\prime}\right)\right) .
\end{aligned}
$$

From the definitions of $s_{\omega}^{+}(\theta)$ and $s_{\theta}^{-}(\omega)$, we obtain $s_{0}=-s_{\theta}^{-}(\omega)$. We put $\tilde{M}_{\theta}^{-}(\omega)=\left\{y^{\prime} \in \partial \mathbb{R}_{+}^{3} ; c_{R}^{-1} \theta \cdot y^{\prime}=s, s \geq c_{R}^{-1} r_{0}, \lim _{t \rightarrow-\infty} p\left(t-s, y^{\prime}, c_{R}^{-1} \theta\right)=c_{R}^{-1} \omega\right\}$ 
and $M_{\theta}^{-, \max }(\omega)=\left\{y^{\prime} \in \tilde{M}_{\theta}^{-}(\omega) ; s_{\theta}^{-}\left(\omega ; y^{\prime}\right)=s_{\theta}^{-}(\omega)\right\}$. From the assumption (1.2) and $H\left(y^{\prime}, \xi\right)=c_{R}|\xi|$ for $\left.\left(y^{\prime}, \xi\right) \in T^{*}(\Gamma)\right|_{\left(B_{r_{0}}\right)}$, we can see that $q\left(t-s-c_{R}^{-1} r_{0}, y^{\prime}+\right.$ $\left.c_{R} s \theta, c_{R}^{-1} \theta\right) \cdot \omega-t$ is independent of $t, s$ and $y^{\prime} \in M_{\theta}^{-}(\omega)$ if $-t(>1)$ is sufficiently large and $s \geq 0$. This implies that $s_{\theta}^{-}(\omega)=\inf _{y^{\prime} \in \tilde{M}_{\theta}^{-}(\omega)} s_{\theta}^{-}\left(\omega ; y^{\prime}\right)=s_{\theta}^{-}\left(\omega ; y^{\prime}\right)$ for any $y^{\prime} \in M_{\theta}^{-, \max }(\omega)$. The sets $U_{j, \epsilon}=\left\{y^{\prime} \in U_{x_{j}^{\prime}} ;\left|s_{\theta}^{-}\left(\omega ; y^{\prime}\right)-s_{\theta}^{-}(\omega)\right|<\epsilon\right\}$ are open neighborhoods of $\operatorname{supp}\left[\psi_{x_{j}^{\prime}}\right] \cap M_{\theta}^{-, \max }(\omega)$ since $s_{\theta}^{-}\left(\omega ; y^{\prime}\right)$ is $C^{\infty}$ in $y^{\prime} \in U_{x_{j}^{\prime}}$.

We put $\tilde{y}^{\prime}(z)=\theta \cdot x_{j}^{\prime} \theta+z \theta^{\perp}, \mathcal{U}_{j, \epsilon}=\left\{z \in \mathbb{R} ; \tilde{y}^{\prime}(z) \in U_{j, \epsilon}\right\}$, and $\mathcal{U}_{j, \epsilon}^{\max }=\{z \in$ $\left.\mathcal{U}_{j, \epsilon} ; \tilde{y}^{\prime}(z) \in M_{\theta}^{-, \max }(\omega)\right\}$. We also define $\tilde{X}(t, z)$ and $t(s, z)$ by $\tilde{X}(t, z)=X\left(t, \tilde{y}^{\prime}(z)\right)$ and $t(s, z)=c_{R}^{-1} \omega \cdot \tilde{X}(s, z)$. Since $\tilde{X}(s, z)=\tilde{X}\left(-s_{0}-t_{1}, z\right)+c_{R}\left(s+s_{0}+t_{1}\right) \Theta\left(\tilde{y}^{\prime}(z)\right)$ for $s \leq-s_{0}-t_{1}, s_{0}+s_{\theta}^{-}\left(\omega ; \tilde{y}^{\prime}(z)\right)=0$ for $z \in \mathcal{U}_{j, \epsilon}^{\max }$ and $\Theta\left(\tilde{y}^{\prime}(z)\right)=\omega$, using (5.27), we have $s=c_{R}^{-1} \omega \cdot \tilde{X}(s, z)$. Thus, if $\epsilon>0$ is chosen to be sufficiently small, we have $0 \leq t(s, z)-s \leq c_{R}^{-1} / 2$ for $z \in \mathcal{U}_{j, \epsilon}$ and $s$ satisfying $-s_{0}-t_{1}-8 c_{R}^{-1} \leq s \leq-s_{0}-t_{1}$. This fact and the equality $h_{+}^{s-\tau_{0}}\left(\tilde{y}^{\prime}(z)+c_{R} r \theta, c_{R}^{-1} \theta\right)=h_{+}^{s+r-\tau_{0}}\left(\tilde{y}^{\prime}(z), c_{R}^{-1} \theta\right)(s, r \in \mathbb{R}$, $\left.|r|<1, z \in \mathcal{U}_{j, \epsilon}\right)$ imply that

$$
X\left(t(s, z), \tilde{y}^{\prime}(z)-c_{R}(t(s, z)-s) \theta\right)=\tilde{X}(s, z) \quad \text { and } \quad t(s, z)=c_{R}^{-1} \omega \cdot \tilde{X}(s, z)
$$

(for $z \in \mathcal{U}_{j, \epsilon}$ and $s$ satisfying $-s_{0}-t_{1}-8 c_{R}^{-1} \leq s \leq-s_{0}-t_{1}$ ).

Since $K_{j}\left(t, y^{\prime}, \omega, \theta\right)=c_{R}^{-1} \omega \cdot X\left(t, y^{\prime}\right)-t\left(\right.$ cf. (5.27)), $\tilde{X}(t, z)=\tilde{X}\left(-s_{0}-t_{1}, z\right)+$ $c_{R}\left(t+s_{0}+t_{1}\right) \Theta\left(\tilde{y}^{\prime}(z)\right)$ for $t \leq-s_{0}-t_{1}$, and $\tilde{X}(t+r, z)=X\left(t, \tilde{y}^{\prime}(z)+c_{R} r \theta\right)$, the solution $y^{\prime}=G(t, z)$ of the equation $K_{j}\left(t, y^{\prime}, \omega, \theta\right)=0$ (in $y^{\prime}$ ) is of the form $G(t, z)=$ $c_{R} g(t, z) \theta+\tilde{y}^{\prime}(z)$, where

$$
\begin{array}{r}
g(t, z)=-s_{0}-t_{1}-t+\frac{1}{\omega \cdot \Theta\left(\tilde{y}^{\prime}(z)\right)}\left\{t-c_{R}^{-1} \omega \cdot \tilde{X}\left(-s_{0}-t_{1}, z\right)\right\} \\
\quad \text { for } z \in \mathcal{U}_{j, \epsilon}=\left\{z \in \mathbb{R} ; \tilde{y}^{\prime}(z) \in U_{j, \epsilon}\right\} .
\end{array}
$$

We put $s(t, z)=t+g(t, z)$. Then, noting that $G(t, z)=c_{R}(t-s(t, z)) \theta+\tilde{y}^{\prime}(z)$ and $X(t, G(t, z))=X\left(t, \tilde{y}^{\prime}(z)-c_{R}(t-s(t, z)) \theta\right)$, we obtain

$$
X\left(t, \tilde{y}^{\prime}(z)-c_{R}(t-s(t, z)) \theta\right)=\tilde{X}(s(t, z), z) \quad \text { and } \quad t=c_{R}^{-1} \omega \cdot \tilde{X}(s(t, z), z) .
$$

From (6.9) and (6.10), it follows that $t\left(s\left(t^{\prime}, z\right), z\right)=c_{R}^{-1} \omega \cdot \tilde{X}\left(s\left(t^{\prime}, z\right), z\right)=t^{\prime}$. We also have $s\left(t\left(s^{\prime}, z\right), z\right)=s^{\prime}$. Indeed, (6.9) and (6.10) imply that $c_{R}^{-1} \omega \cdot \tilde{X}\left(s^{\prime}, z\right)=t\left(s^{\prime}, z\right)=$ $c_{R}^{-1} \omega \cdot \tilde{X}(\tilde{s}, z)$ for $\tilde{s}=s\left(t\left(s^{\prime}, z\right), z\right)$. Since $\tilde{X}\left(s^{\prime}, z\right)=\tilde{X}\left(-s_{0}-t_{1}, z\right)+c_{R}\left(s^{\prime}+s_{0}+\right.$ $\left.t_{1}\right) \Theta\left(\tilde{y}^{\prime}(z)\right)$ and $\tilde{X}(\tilde{s}, z)=\tilde{X}\left(-s_{0}-t_{1}, z\right)+c_{R}\left(\tilde{s}+s_{0}+t_{1}\right) \Theta\left(\tilde{y}^{\prime}(z)\right)$, we obtain $\tilde{s}=s^{\prime}$.

Now we show that the map $\tilde{X}:\left(-\infty,-s_{0}-t_{1}\right] \times \cup_{j=1}^{N_{1}} \mathcal{U}_{j, \epsilon} \rightarrow\left(B_{r_{0}}\right)^{c}$ is injective if $\epsilon>0$ is sufficiently small. It suffices to show that $t=t^{\prime}$ and $z=z^{\prime}$ for any $t, t^{\prime} \leq$ $-s_{0}-t_{1}$ and $z, z^{\prime} \in \cup_{j=1}^{N_{1}} \mathcal{U}_{j, \epsilon}^{\max }$ that satisfies $\tilde{X}(t, z)=\tilde{X}\left(t^{\prime}, z^{\prime}\right)$. If this is the case, note that $t=c_{R}^{-1} \omega \cdot \tilde{X}(t, z)=c_{R}^{-1} \omega \cdot \tilde{X}\left(t^{\prime}, z\right)=t^{\prime}$, i.e., $t=t^{\prime}$. Set $x^{\prime}=\tilde{X}(t, z)=\tilde{X}\left(t^{\prime}, z^{\prime}\right)$. Then, noting that $h^{s-t}\left(x^{\prime}, c_{R}^{-1} \omega\right)=h^{s-\tau_{0}}\left(\tilde{y}^{\prime}(z), c_{R}^{-1} \theta\right)(s \in \mathbb{R})$, we have $z=z^{\prime}$.

Lemma 6.1. Let $s \leq-s_{0}-t_{1}$ and $z \in \mathcal{U}_{j, \epsilon}\left(j=1, \ldots, N_{1}\right)$. Then, we obtain

$$
\begin{aligned}
& \left.\left\{S_{j}\left(c_{R}^{-1} \omega \cdot x^{\prime}, x^{\prime}\right)-c_{R}^{-1} \omega \cdot x^{\prime}\right\}\right|_{x^{\prime}=\tilde{X}(s, z)}=s_{0}+s-t(s, z), \\
& \left|\operatorname{det} \frac{\partial X}{\partial y^{\prime}}(t, G(t, z))\right|=\left|\operatorname{det} \frac{\partial \tilde{X}}{\partial(t, z)}(t, z)\right|=\left|\frac{\partial \tilde{X}}{\partial z}(t, z)\right| .
\end{aligned}
$$

Proof. The restriction of the canonical 1-form $p \cdot d q$ on $\mathcal{L}_{T}^{3,+}\left(U_{x_{j}^{\prime}}\right)$ is represented by the form $\left(p \cdot \partial_{t} q\right) d t+\sum_{k=1}^{2}\left(p \cdot \partial_{y_{k}} q\right) d y_{k}$ in the coordinate of $\mathcal{L}_{T}^{3,+}\left(U_{x_{j}^{\prime}}\right)$ given by 
$\left(t, y^{\prime}\right) \mapsto r^{+}\left(t, y^{\prime}\right)$. Since the tangent vector $\dot{r}^{+}\left(t, y^{\prime}\right)\left(=\frac{\partial}{\partial t} r^{+}\left(t, y^{\prime}\right)\right)$ means $\frac{\partial}{\partial t}$ in the coordinate $\left(t, y^{\prime}\right), p \cdot d q\left(\dot{r}^{+}\left(t, y^{\prime}\right)\right)$ is equal to $p \cdot \partial_{t} q\left(r^{+}\left(t, y^{\prime}\right)\right)$. Noting that $\dot{r}^{+}\left(t, y^{\prime}\right)$ is represented by $\dot{r}^{+}\left(t, y^{\prime}\right)={ }^{t}\left(1,0, H_{p},-H_{q}\right)$ in every canonical coordinate, we get $p \cdot d q\left(\dot{r}^{+}\left(t, y^{\prime}\right)\right)=p \cdot H_{p}=H=1$ since $H$ is homogeneous of order 1 with respect to $p$ and $H(q, p)=H\left(y^{\prime}, c_{R}^{-1} \theta\right)=1$. Thus, we obtain $p \cdot \partial_{t} q\left(r^{+}\left(t, y^{\prime}\right)\right)=1$ for any $\left(t, y^{\prime}\right)$. This fact and (5.24) imply that $S_{j}\left(t, y^{\prime}\right)=c_{R}^{-1} \theta \cdot y^{\prime}+t-\tau_{0}$. Hence, from (6.9) we have

$$
\begin{aligned}
& \left.\left\{S_{j}\left(c_{R}^{-1} \omega \cdot x^{\prime}, x^{\prime}\right)-c_{R}^{-1} \omega \cdot x^{\prime}\right\}\right|_{x^{\prime}=\tilde{X}(s, z)}=\left.\left\{\tilde{S}_{j}(t, \tilde{X}(s, z))-t\right\}\right|_{t=c_{R}^{-1} \omega \cdot \tilde{X}(s, z)} \\
& \quad=S_{j}\left(t(s, z), X\left(t(s, z), \tilde{y}^{\prime}(z)-c_{R}(t(s, z)-s) \theta\right)\right)-t(s, z)=s_{0}+s-t(s, z) .
\end{aligned}
$$

Next, we show (ii). Since $p \cdot d q$ is a closed form on $\mathcal{L}_{T}^{3,+}\left(U_{x_{j}^{\prime}}\right)$, we have $\partial_{y_{k}}\left(p \cdot \partial_{t} q\right)=$ $\partial_{t}\left(p \cdot \partial_{y_{k}} q\right)$ for $k=1,2$. Hence, $p \cdot \partial_{y_{k}} q$ are independent of $t$, which implies that

$$
\frac{\partial \tilde{X}}{\partial z}(t, z) \cdot \Theta\left(\tilde{y}^{\prime}(z)\right)=0
$$

since $\left.h_{+}^{t-\tau_{0}}\left(\tilde{y}^{\prime}(z), c_{R}^{-1} \theta\right)\right|_{t=\tau_{0}}=\left(\tilde{y}^{\prime}(z), c_{R}^{-1} \theta\right)$. Differentiating (6.10) in $z$ and noting that $\partial_{s} \tilde{X}(s, z)=c_{R} \Theta\left(\tilde{y}^{\prime}(z)\right)$, we obtain

$$
\frac{\partial X}{\partial y^{\prime}}\left(\theta^{\perp}+\left(\partial_{z} s\right) \theta\right)=c_{R}\left(\partial_{z} s\right) \Theta\left(\tilde{y}^{\prime}(z)\right)+\partial_{z} \tilde{X}
$$

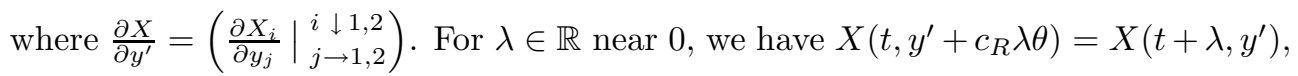
which yields

$$
\frac{\partial X}{\partial y^{\prime}} \theta=\Theta\left(\tilde{y}^{\prime}(z)\right)
$$

From (6.12) and (6.13), it follows that

$$
\frac{\partial X}{\partial y^{\prime}}\left(\theta, \theta^{\perp}+\left(\partial_{z} s\right) \theta\right)=\left(\Theta\left(\tilde{y}^{\prime}(z)\right), c_{R}\left(\partial_{z} s\right) \Theta\left(\tilde{y}^{\prime}(z)\right)+\partial_{z} \tilde{X}\right),
$$

which implies that

$$
\begin{aligned}
\left|\operatorname{det} \frac{\partial X}{\partial y^{\prime}}(t, G(t, z))\right|^{2} & =\left|\operatorname{det}\left(\Theta\left(\tilde{y}^{\prime}(z)\right), \partial_{z} \tilde{X}\right)\right|^{2} \\
& =\left|\operatorname{det}\left\{{ }^{t}\left(\Theta\left(\tilde{y}^{\prime}(z)\right), \partial_{z} \tilde{X}\right)\left(\Theta\left(\tilde{y}^{\prime}(z)\right), \partial_{z} \tilde{X}\right)\right\}\right| .
\end{aligned}
$$

From this equality and (6.11), we obtain (ii) of Lemma 6.1.

We change the variable $x^{\prime}=\tilde{X}(s, z)$ in the integral (6.8). Since supp $\left[\phi_{2} \partial_{t} \phi_{1}\right]$ $\subset\left[-s_{0}-t_{1}-2 c_{R}^{-1},-s_{0}-t_{1}-c_{R}^{-1}\right]$, it follows from Lemma 6.1 that

$$
I_{j, q}(\varphi)=\int_{\mathbb{R}} d \tau \int_{\mathbb{R}} d s \int_{\mathcal{U}_{j, \epsilon}} d z e^{i \tau\left(s_{0}+s-t(s, z)\right)} \eta(s, z) \beta_{j, q}(s, z, \tau)(i \tau)^{1-q} \phi_{7}(\tau) \hat{\varphi}(\tau),
$$

where $\beta_{j, q}(s, z, \tau)=\tilde{b}_{j, q}\left(r^{ \pm}\left(t(s, z), \tilde{y}^{\prime}(z)\right), \tau\right)\left|\partial_{z} \tilde{X}(s, z)\right|(\tau \neq 0)$ and $\eta(s, z) \stackrel{(6.14)}{=}$ $\phi_{2}(t(s, z)) \partial_{t} \phi_{1}(t(s, z)) \tilde{\psi}(\tilde{X}(s, z))$. 
Note that the subprincipal symbol $\sigma_{s p}\left(\lambda^{(e)}\right)$ of $O p\left(\chi_{1} \lambda^{(e)}\right)_{\Omega^{\frac{1}{2}}} \in \Psi_{p h g}^{1}(\mathbb{R} \times$ $\left.\Gamma ; \Omega^{\frac{1}{2}}, \Omega^{\frac{1}{2}}\right)$ is a real-valued function on $\left\{(t, \tau, \zeta) \in T^{*}(\mathbb{R} \times \Gamma) ; \chi_{1}(t, \tau, \zeta)=1\right\}$. Indeed, multiplying $\left(\tilde{E}^{(0)}\right)^{*}$ (resp. $\left.\tilde{E}^{(0)}\right)$ to $(5.2)$ from the left (resp. right) side, and noting that (i) of Proposition 5.4, we have $\left(\tilde{E}^{*} O p\left(\chi_{1}\right) \tilde{E}^{(0)}\right)^{*} O p\left(\chi_{1} \lambda^{(e)}\right)-$ $\left(O p\left(\chi_{1} \lambda^{(e)}\right)\right)^{*} \tilde{E}^{*} O p\left(\chi_{1}\right) \tilde{E}^{(0)} \in \Psi^{-\infty}(\mathbb{R} \times \Gamma)$. Since $\sigma_{p}\left(\tilde{E}^{*}\right) \sigma_{p}\left(\tilde{E}-\tilde{E}^{(0)}\right)=0$ yields $\tilde{E}^{*} O p\left(\chi_{1}\right)\left(\tilde{E}-\tilde{E}^{(0)}\right) \in \Psi_{p h g}^{-2}(\mathbb{R} \times \Gamma)$, we obtain

$$
\left(\tilde{E}^{*} O p\left(\chi_{1}\right) \tilde{E}\right)^{*} O p\left(\chi_{1} \lambda^{(e)}\right)-\left(O p\left(\chi_{1} \lambda^{(e)}\right)\right)^{*} \tilde{E}^{*} O p\left(\chi_{1}\right) \tilde{E} \in \Psi_{p h g}^{-1}(\mathbb{R} \times \Gamma) .
$$

This fact and Proposition 5.1 imply $\sigma_{s p}\left(\lambda^{(e)}\right)=\overline{\sigma_{s p}\left(\lambda^{(e)}\right)}$ on $\left\{(t, \tau, \zeta) \in T^{*}(\mathbb{R} \times\right.$ $\left.\Gamma) ; \chi_{1}(t, \tau, \zeta)=1\right\}$.

Since $\check{I}_{\mathbb{R} \times \Gamma} \lambda_{j}^{(e)}(\tau, \zeta)=\overline{\lambda_{j}^{(e)}(\tau, \zeta)}(j=0,1)$, we have $\check{I}_{\mathbb{R} \times \Gamma} \sigma_{s p}\left(\lambda^{(e)}\right)=$ $\overline{\sigma_{s p}\left(\lambda^{(e)}\right)}=\sigma_{s p}\left(\lambda^{(e)}\right)$ on $\left\{(t, \tau, \zeta) \in T^{*}(\mathbb{R} \times \Gamma) ; \chi_{1}(t, \tau, \zeta)=1\right\}$, which yields

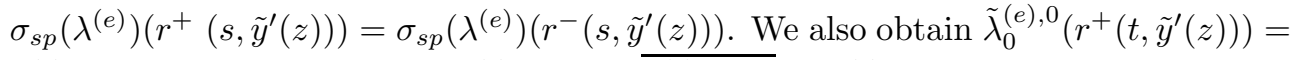
$\tilde{\lambda}_{0}^{(e), 0}\left(r^{-}\left(t, \tilde{y}^{\prime}(z)\right)\right)$ since $\check{I}_{\mathbb{R} \times \Gamma} \lambda_{0}^{(e)}(\tau, \zeta)=\overline{\lambda_{0}^{(e)}(\tau, \zeta)}=\lambda_{0}^{(e)}(\tau, \zeta)$ and because of (i) of Proposition 5.1. Therefore, combining (5.11), (5.19), (6.6), (6.4) and the equality $\phi_{6} \phi_{2} \partial_{t} \phi_{1}=\phi_{2} \partial_{t} \phi_{1}$, we get $\beta_{j, 0}(s, z, \tau)=\tilde{\beta}_{j, 0}(s, z) \phi_{7}(\tau)$, where

$$
\begin{gathered}
\tilde{\beta}_{j, 0}(s, z)=-2 i \rho_{0} c_{R} \phi_{0}(t(s, z)) \psi_{1}(\tilde{X}(s, z)) \phi_{5}\left(s_{0}+s-t(s, z)\right)\left(1+\omega \cdot \Theta\left(\tilde{y}^{\prime}(z)\right)\right) \\
\cdot e^{-i \pi m_{j} / 2} \int_{s}^{\infty} \psi_{x_{j}^{\prime}}\left(\tilde{y}^{\prime}(z)+c_{R} \lambda \theta\right) \partial_{\lambda}\left(\tilde{\psi}\left(\tilde{y}^{\prime}(z)+c_{R} \lambda \theta\right)\right) d \lambda \\
\cdot \exp \left(i \int_{s}^{\infty} \sigma_{s p}\left(\lambda^{(e)}\left(r^{+}\left(\lambda, \tilde{y}^{\prime}(z)\right)\right)\right) d \lambda\right)\left|\partial_{z} \tilde{X}(s, z)\right|^{1 / 2}
\end{gathered}
$$

Lemma 6.2. Set $\Phi(s, z)=s_{0}+s-t(s, z)$, and let $\epsilon>0$ be sufficiently small. Then, we obtain the following (i) and (ii) for each $j\left(=1, \ldots, N_{1}\right)$ :

(i) For any fixed $s \leq-s_{0}-t_{1}$, we have $\partial_{z} \Phi(s, z)=0$ if and only if $z \in \mathcal{U}_{j, \epsilon}^{\max }$ (i.e., the stationary points of $\Phi$ in $z$ are characterized by $\left.z \in \mathcal{U}_{j, \epsilon}^{\max }\right)$.

(ii) For any fixed $s \leq-s_{0}-t_{1}$, we have $\Phi(s, z)=s_{0}\left(=s_{\omega}^{+}(\theta)\right)$ if $\partial_{z} \Phi(s, z)=0$, i.e., $z \in \mathcal{U}_{j, \epsilon}^{\max }$.

Note that (i) and (ii) of this Lemma imply that $\partial_{(s, z)} \Phi(s, z)=0$ for $s \leq-s_{0}-t_{1}$, $z \in \mathcal{U}_{j, \epsilon}$ if and only if $z \in \mathcal{U}_{j, \epsilon}^{\max }$.

Proof of Lemma 6.2. Note that $\partial_{z} \Phi(s, z)=0$ is equivalent to $\omega \cdot \partial_{z} \tilde{X}(\tilde{s}, z)=0$. This fact and (6.11) imply that $\left\{\Theta\left(\tilde{y}^{\prime}(z)\right), \omega\right\}$ is linearly dependent if $\partial_{z} \tilde{X}(s, z) \neq$ 0 is verified. This follows from the equality $\partial_{z} \tilde{X}(s, z)=\frac{\partial X}{\partial y^{\prime}}\left(s, \tilde{y}^{\prime}(z)\right) \theta^{\perp}$ and local diffeomorphism of the map: $y^{\prime} \mapsto X\left(s, y^{\prime}\right)$. Thus, we obtain (i) of Lemma 6.2 if $\epsilon>0$ is chosen to be sufficiently small. For $z_{0} \in \mathcal{U}_{j, \epsilon}^{\max },(5.26)$ implies $-s_{0}=c_{R}^{-1} \omega$. $\tilde{X}\left(s, z_{0}\right)-\left(s+s_{0}\right)$, which means (ii). Thus Lemma 6.2 is proved.

From Theorem 3.1, (6.1), (6.7), (6.14) and (6.15), we can see that $\tilde{S}_{R R}(s, \theta, \omega)=$ $2(-2 \pi i)^{2} c_{R}^{3} \rho_{0} S_{R R}(s, \theta, \omega)$ is reduced to a distribution $K_{0} \in \mathcal{D}^{\prime}(\mathbb{R})$ near $s=s_{0}$ modulo smooth function, i.e.

$$
\left\langle\tilde{S}_{\mathrm{RR}}(\cdot, \theta, \omega), \varphi\right\rangle \simeq K_{0}(\varphi) \quad \text { with respect to } \quad C_{0}^{\infty}(I) \rightarrow \mathbb{C} \quad\left(\varphi \in C_{0}^{\infty}(I)\right),
$$


where $K_{0}$ is defined by oscillatory integrals of the form

$$
K_{0}(\varphi)=(2 \pi)^{-1} \int_{\mathbb{R}} \int_{\mathcal{U}} e^{i \tau \Phi(s, z)} \eta(s, z) \beta(s, z, \tau) \hat{\varphi}(\tau) d x d \tau, \quad \varphi \in C_{0}^{\infty}(I) .
$$

In (6.16), $\mathcal{U}=\cup_{j=1}^{N_{1}} \mathcal{U}_{j, \epsilon}$ and $\beta(s, z, \tau) \in S_{p h g}^{1}\left(\mathbb{R}_{(s, z)}^{2} \times \mathbb{R}_{\tau}\right)$ is an amplitude function that satisfies

$$
\begin{aligned}
\beta(s, z, \tau) & \sim \beta_{0}(s, z)(i \tau)+\sum_{q=0}^{\infty} \beta_{q}(s, z, \tau), \\
\beta_{0}(s, z) & =\sum_{j=1}^{N_{1}} \tilde{\beta}_{j, 0}(s, z), \quad \beta_{q}(s, z, \tau)=\sum_{j=1}^{N_{1}} \beta_{j, q}(s, z, \tau)(i \tau)^{1-q} \quad(q \geq 1) .
\end{aligned}
$$

Note that each $\beta_{q}(q \geq 1)$ is homogeneous of order $1-q$ in $\tau$. Thus, the proof of Theorem 1.2 is reduced to studying the singularities of some class of distribution that is defined by an oscillatory integral of the form (6.16) with the phase function $\Phi(s, z)$ and amplitude function $\beta(s, z, \tau)$ having the properties described in Lemma 6.2 and in the above, respectively.

In the section 7 , we consider the oscillatory integrals that appear above (cf. Theorems 7.1 and 7.2). From Theorem 7.2, we can immediately obtain the following theorem, which provides more precise results than Theorem 1.2.

TheOrem 6.3. Assume that there exists an $\tilde{\omega} \in \mathbb{C}$ with $|\tilde{\omega}|=1$ such that $\operatorname{Re} \tilde{\omega} \beta_{0}(s, z)>0$ in $(s, z) \in\left[-s_{0}-t_{1}-2 c_{R}^{-1},-s_{0}-t_{1}-c_{R}^{-1}\right] \times \mathcal{U}_{j, \epsilon}^{\max }$. Then, we obtain the following (i) and (ii):

(i) $S_{\mathrm{RR}}(\cdot, \theta, \omega) \notin H^{-1} \quad$ at $s=s_{0}$.

(ii) We assume that there exists $l \in \mathbb{N}$ such that $\partial_{z}^{p} \Phi(s, z)=0$ for all $p=1,2, \ldots, l-1$, $z \in \cup_{j=1}^{N_{1}} \mathcal{U}_{j, \epsilon}^{\max }$ and $s$ satisfying $-s_{0}-t_{1}-2 c_{R}^{-1^{2}} \leq s \leq-s_{0}-t_{1}-c_{R}^{-1}$, and assume that $\partial_{z}^{l} \Phi\left(s, z_{0}\right) \neq 0$ for any $z_{0} \in \cup_{j=1}^{N_{1}} \mathcal{U}_{j, \epsilon}^{\max }$. Then, $l$ is even, and we obtain

$$
\begin{array}{lll}
S_{\mathrm{RR}}(\cdot, \theta, \omega) \notin H^{-\frac{3}{2}+\frac{1}{l}} & \text { at } s=s_{0}, & \text { and } \\
S_{\mathrm{RR}}(\cdot, \theta, \omega) \in H^{-\frac{3}{2}+\frac{1}{l}-\epsilon_{0}} & \text { at } s=s_{0} & \left(\text { for any } \epsilon_{0}>0\right) .
\end{array}
$$

Note that $l$ in (ii) of Theorem 6.3 is even since $\Phi(s, z)$ takes the maximum at $\left(s, z_{0}\right)$.

Proof of Theorem 1.2. The assumption in Theorem 1.2 means that we have only one $j$ with $\mathcal{U}_{j, \epsilon}^{\max } \neq \emptyset$. Furthermore, this set consists of only one point $z_{0}$. From (6.15), it follows that

$$
\begin{aligned}
\tilde{\beta}_{j, 0}\left(s, z_{0}\right)=-4 i \rho_{0} c_{R} \exp \left(i \int_{-\infty}^{\infty} \sigma_{s p}\left(\lambda^{(e)}\left(r^{+}\left(\lambda, \tilde{y}^{\prime}\left(z_{0}\right)\right)\right)\right) d \lambda\right)\left|\partial_{z} \tilde{X}\left(s, z_{0}\right)\right|^{1 / 2} & \\
& \text { in } s \in\left[-s_{0}-t_{1}-2 c_{R}^{-1},-s_{0}-t_{1}-c_{R}^{-1}\right] .
\end{aligned}
$$

This implies that the assumption of Theorem 6.3 is satisfied. Hence we obtain Theorem 1.2.

Next, we consider the case that every stationary point of $\Phi$ is "non-degenerate". From now on, we assume the following "non-degenerate condition".

$$
\frac{\partial}{\partial z} \Theta\left(\tilde{y}^{\prime}(z)\right) \neq 0 \quad \text { (for any } z \in \cup_{j=1}^{N_{1}} \mathcal{U}_{j, \epsilon}^{\max } \text { ). }
$$


In this case, using (6.11), we have

$$
\partial_{z}^{2} \Phi\left(s, z_{0}\right)=-c_{R}^{-1} \omega \cdot \partial_{z}^{2} \tilde{X}\left(s, z_{0}\right)=c_{R}^{-1} \frac{\partial}{\partial z} \Theta\left(\tilde{y}^{\prime}\left(z_{0}\right)\right) \cdot \frac{\partial \tilde{X}}{\partial z}\left(s, z_{0}\right) \neq 0
$$

for any $z_{0} \in \cup_{j=1}^{N_{1}} \mathcal{U}_{j, \epsilon}^{\max }$. This means that $\cup_{j=1}^{N_{1}} \mathcal{U}_{j, \epsilon}^{\max }$ is a finite set. Let $\cup_{j=1}^{N_{1}} \mathcal{U}_{j, \epsilon}^{\max }=$ $\left\{z_{1}, z_{2}, \ldots, z_{N_{2}}\right\}$. From $\tilde{y}^{\prime}\left(z_{0}\right) \in M_{\theta}^{-,}, \max (\omega)$, it follows that $t(s, z) \geq t\left(s, z_{0}\right)=s$ in $z \in \cup_{j=1}^{N_{1}} \mathcal{U}_{j, \epsilon}$. Hence, we get $\partial_{z}^{2} t\left(s, z_{0}\right) \geq 0$ since $s+\partial_{z}^{2} t\left(s, z_{0}\right)\left(z-z_{0}\right)^{2}+O\left(\left|z-z_{0}\right|^{3}\right) \geq s$. From this fact and (6.18), we have

$$
\partial_{z}^{2} \Phi\left(s, z_{j}\right)<0 \quad j=1,2, \ldots, N_{2} .
$$

We introduce a function $\lambda_{+}(\tau)$ defined by

$$
\lambda_{+}(\tau)= \begin{cases}e^{-i \pi / 4} \tau^{1 / 2} & (\tau>0) \\ e^{i \pi / 4}|\tau|^{1 / 2} & (\tau<0)\end{cases}
$$

Theorem 6.4. Assume (1.2) and (6.17). Then, we obtain

$$
\left\langle\tilde{S}_{\mathrm{RR}}(\cdot, \theta, \omega), \varphi\right\rangle=\sum_{r=1}^{N_{2}} \sum_{p=1}^{N} Q_{p, r}(\varphi)+R_{N}(\varphi),
$$

where $R_{N} \in \mathcal{D}^{\prime}\left(\left(s_{\omega}^{+}(\theta)-\epsilon, s_{\omega}^{+}(\theta)+\epsilon\right)\right)$ satisfies $\left|R_{N}(\varphi)\right| \leq C_{N}\|\varphi\|_{H^{1 / 2-N}(\mathbb{R})}$, and each $Q_{p, r}(\varphi)$ is given by

$$
Q_{p, r}(\varphi)=C_{p, r}\left(D_{s}\right) \lambda_{+}\left(D_{s}\right)^{*} \varphi\left(s_{\omega}^{+}(\theta)\right) .
$$

Here, $C_{p, r}(\tau) \in S^{-p}(\mathbb{R})$ is homogeneous of order $-p(|\tau|>1)$, and $C_{0, r}$ is a constant given by

$$
\begin{aligned}
C_{0, r}(\tau)=-(2 \pi)^{3 / 2} i \rho_{0} c_{R}^{2} e^{-i \pi \tilde{m}_{r} / 2}\left|\left(\frac{\partial}{\partial z} \Theta\left(\tilde{y}^{\prime}\left(z_{r}\right)\right)\right)\right|^{-1 / 2} \\
\quad \exp \left(i \int_{-\infty}^{\infty} \sigma_{s p}\left(\lambda^{(e)}\right)\left(r^{+}\left(\lambda, \tilde{y}^{\prime}\left(z_{r}\right)\right)\right) d \lambda\right),
\end{aligned}
$$

where $\tilde{m}_{r}$ is the Morse index of the path $s \mapsto \pi_{\Gamma}\left(r\left(s, \tilde{y}^{\prime}\left(z_{r}\right)\right)\right)$.

Thus, in the non-degenerate case (6.17), we can see $S_{\mathrm{RR}}(\cdot, \theta, \omega) \notin H^{-1}$, and for any $\epsilon_{0}>0, S_{\mathrm{RR}}(\cdot, \theta, \omega) \in H^{-1-\epsilon_{0}}$ at $s=s_{\omega}^{+}(\theta)$. These results are consistent with Theorem 6.3. Concerning weakness of the singularities of $S_{\mathrm{RR}}(\cdot, \theta, \omega)$ at the maximal sojourn time $s_{\omega}^{+}(\theta)$, we can say that the non-degenerate case is the weakest one.

Proof of Theorem 6.4. It suffices to consider the case of the one stationary point $z_{0} \in \mathcal{U}_{j, \epsilon}^{\max }$ since the stationary points are isolated. Based on (6.18) and (6.19), the method of stationary phase yields that

$$
\begin{aligned}
\int_{\mathbb{R}} e^{i \tau \Phi(s, z)} & \eta(s, z) \beta_{j, q}(s, z, \tau) d z \\
& =e^{-i 4 \pi \tau|\tau|^{-1}} e^{i \tau s_{0}}|\tau|^{-1 / 2}\left\{\sum_{p=0}^{N-1} \tilde{C}_{j q p}(s, \tau)(i \tau)^{-p}+\tilde{R}_{N, j q}(s, \tau)|\tau|^{-N}\right\}
\end{aligned}
$$


where $\tilde{C}_{j q p}(s, \tau)$ and $\tilde{R}_{N, j q}(s, \tau)\left(\in S^{0}\left(\mathbb{R}_{s} \times \mathbb{R}_{\tau}\right)\right)$ are homogeneous of order 0 , and $\tilde{C}_{j q 0}(s, \tau)=(2 \pi)^{1 / 2} \eta\left(s, z_{0}\right) \beta_{j q}\left(s, z_{0}, \tau\right)\left|\partial_{z}^{2} \Phi\left(s, z_{0}\right)\right|^{-1 / 2}$. Since we have $\exp \left(-i \pi \tau|\tau|^{-1} / 4\right) i \tau|\tau|^{-1 / 2}=\overline{\lambda_{+}(\tau)}$, it follows from (6.20) and (6.14) that

$$
I_{j q}(\varphi)=\sum_{p=0}^{N-1} \int_{\mathbb{R}} \int_{\mathbb{R}} \tilde{C}_{j q p}(s, \tau)(i \tau)^{-p-q} \overline{\lambda_{+}(\tau)} e^{i s_{0} \tau} \hat{\varphi}(\tau) d \tau d s+\tilde{R}_{N, j q}(\varphi),
$$

where $\tilde{R}_{N, j q}$ are the Fourier multiplier operators homogeneous of order $-N+1 / 2-q$. Combining this fact, (6.7), (6.8), (6.18) and (6.15), we obtain Theorem 6.4.

7. Singularities of distributions defined by oscillatory integrals. In this section, we examine the singularities of the distributions given by (6.16). We introduce a class of distributions on $\mathbb{R}^{n}$ containing the one defined by (6.16) in $n$-dimensional space. We change the notations since the contents of this section can be treated independently of the previous sections.

Let $S(x)$ be a real-valued $C^{\infty}$ function on an open set $U \subset \mathbb{R}^{n}$, and let $\beta(x, \tau) \in$ $S_{p h g}^{m}\left(U \times \mathbb{R}_{\tau}\right)(m \in \mathbb{N} \cup\{0\})$ be a symbol of the form

$$
\beta(x, \tau) \sim \beta_{0}(x)(i \tau)^{m}+\sum_{j=1}^{\infty} \beta_{j}(x, \tau),
$$

where each $\beta_{j}(x, \tau)$ is homogeneous of order $m-j$ in $\tau$. We examine the distributions $K$ on an open interval $I \subset \mathbb{R}$ (i.e. $K \in \mathcal{D}^{\prime}(I)$ ) that are defined by the oscillatory integral

$$
K(\varphi)=(2 \pi)^{-1} \int_{\mathbb{R}} \int_{U} e^{i \tau S(x)} \eta(x) \beta(x, \tau) \hat{\varphi}(\tau) d x d \tau, \quad \varphi \in C_{0}^{\infty}(I),
$$

where $\eta$ is a real-valued function belonging to $C_{0}^{\infty}(U)$. As is described in section 6 , the proof of the main theorems has been reduced to examining the distributions.

We take a bounded open set $V \subset U$ with supp $[\eta] \subset V$, and put $E_{\lambda}=\{x \in$ $\bar{V} ; S(x) \geq \lambda\}$ for $\lambda \in \mathbb{R}, \lambda_{-\infty}=\inf _{x \in \bar{V}} S(x)$ and $\lambda_{\infty}=\sup _{x \in \bar{V}} S(x)$. Hereafter, we assume that

$$
\begin{aligned}
& \lambda_{-\infty}<\lambda_{\infty}, \text { and } \operatorname{Re} \beta_{0}(x)>0 \text { on } E_{\lambda_{\infty}}, \\
& \eta(x) \geq 0 \text { on } U \text { and } \eta\left(x_{0}\right)>0 \text { for some point } x_{0} \in E_{\lambda_{\infty}} .
\end{aligned}
$$

TheOrem 7.1. Assume (7.1) and (7.2) as shown above. Then there exists an integer $k_{0}$ with $0 \leq k_{0} \leq n$ such that we have $K \notin H^{-m+\frac{n-1}{2}-\frac{k_{0}}{4}}$ at $s=\lambda_{\infty}$.

If the assumption in Theorem 6.3 holds, then for the distribution $K_{0}$ introduced by (6.16), $\tilde{\omega} K_{0}$ satisfies (7.1) and (7.2) with $n=2, m=1, \lambda_{\infty}=s_{0}\left(=s_{\omega}^{+}(\theta)\right)$, and $E_{\lambda_{\infty}}=\left[-s_{0}-t_{1}-2 c_{R}^{-1},-s_{0}-t_{1}-c_{R}^{-1}\right] \times \cup_{j=1}^{N_{1}} \mathcal{U}_{j, \epsilon}^{\max }$. As shown in Lemma 6.2, the phase function $\Phi(s, z)$ has stationary points along the lines $s \leq-s_{0}-t_{1}, z \in \mathcal{U}_{j, \epsilon}^{\text {max }}$. This property is stated as follows:

There exists a non-negative integer $k_{1} \leq n-1$ such that for any $x^{(1)} \in E_{\lambda_{\infty}}$ all the points $x^{(1)}+{ }^{t}\left(0,{ }^{t} x^{\prime \prime}\right)$ with $\left|x^{\prime \prime}\right|<\delta_{x^{(1)}}$ belong to $E_{\lambda_{\infty}}$ for some $\delta_{x^{(1)}}>0$, where $x={ }^{t}\left({ }^{t} x^{\prime},{ }^{t} x^{\prime \prime}\right), x^{\prime} \in \mathbb{R}^{n-k_{1}}$ and $x^{\prime \prime} \in \mathbb{R}^{k_{1}}$ (if $k_{1} \geq 1$ ). 
Using this property, we can obtain more precise results.

TheOREM 7.2. Assume (7.1)-(7.3) as shown above. Then, we obtain the following (i) and (ii):

(i) $K \notin H^{-m+\frac{n-k_{1}}{2\left(l_{0}+1\right)}-\frac{1}{2}}$ at $s=\lambda_{\infty}$ if there exist an integer $l_{0}(\geq 0)$ and a positive constant $\delta_{x_{0}}$ such that we have $\partial_{x^{\prime}}^{\alpha^{\prime}} S\left(x_{0}^{\prime}, x_{0}^{\prime \prime}+x^{\prime \prime}\right)=0$ for $0<\left|\alpha^{\prime}\right| \leq 2 l_{0}$ and $\left|x^{\prime \prime}\right|<\delta_{x_{0}}$. (ii) In (i), also assume additionally that $x^{\prime \prime} \in \mathbb{R}^{n-1}$ (i.e. $k_{1}=n-1$ ) and $\partial_{x_{1}}^{2\left(l_{0}+1\right)} S(x) \neq 0$ for any $x \in E_{\lambda_{\infty}}$. Then, for any $\epsilon_{0}>0$ we get $K \in$ $H^{-m+\frac{n-k_{1}}{2\left(l_{0}+1\right)}-\frac{1}{2}-\epsilon_{0}}$ at $s=\lambda_{\infty}$.

Applying Theorem 7.2 with $2 l_{0}=l-2$, we obtain Theorem 6.3.

To show Theorems 7.1 and 7.2 , we begin by decomposing the functional $K$. For any integer $N>m+n+2$, we put

$$
K_{N}(\varphi)=(2 \pi)^{-1} \int_{\mathbb{R}} \int_{U} e^{i \tau S(x)} \eta(x)\left(\beta_{0}(x)(i \tau)^{m}+\sum_{j=1}^{N} \beta_{j}(x, \tau)\right) \hat{\varphi}(\tau) d x d \tau
$$

and $R_{N}(\varphi)=K(\varphi)-K_{N}(\varphi)$. Since $\left|R_{N}(\varphi)\right| \leq C_{N}\|\varphi\|_{H^{-N+m+2}}\left(\varphi \in C_{0}^{\infty}(\mathbb{R})\right)$, it suffices to consider $K_{N}$.

For $\lambda \in \mathbb{R}, 0 \neq \tau \in \mathbb{R}$, we put

$$
\tilde{\beta}_{0}(\lambda)=\int_{E_{\lambda}} \eta(x) \beta_{0}(x) d x \quad \text { and } \quad \tilde{\beta}_{j}(\lambda, \tau)=\int_{E_{\lambda}} \eta(x) \beta_{j}(x, \tau) d x .
$$

Then using the Stieltjes integral with respect to $\tilde{\beta}_{j}$, we have

$$
\begin{aligned}
\int_{U} e^{i \tau S(x)} \eta(x) \beta_{j}(x, \tau) d x & =\int_{\lambda_{-\infty}}^{\lambda_{\infty}} e^{i \tau \lambda} d \tilde{\beta}_{j}(\lambda, \tau) \\
& =-e^{i \tau \lambda_{-\infty}} \tilde{\beta}_{j}\left(\lambda_{-\infty}, \tau\right)-\int_{\lambda_{-\infty}}^{\lambda_{\infty}} e^{i \tau \lambda} i \tau \tilde{\beta}_{j}(\lambda, \tau) d \lambda .
\end{aligned}
$$

To show (7.4), we introduce $\beta_{j}^{R, \pm}(x, \tau)=\max \left\{ \pm \operatorname{Re} \beta_{j}(x, \tau), 0\right\}$ and $\beta_{j}^{I, \pm}(x, \tau)=$ $\max \left\{ \pm \operatorname{Im} \beta_{j}(x, \tau), 0\right\}$ and put $\widetilde{\beta}_{j}^{\alpha, \pm}(\lambda, \tau)=\int_{E_{\lambda}} \eta(x) \beta_{j}^{\alpha, \pm}(x, \tau) d x(\alpha=R, I)$. Then the functions $\tilde{\beta}_{j}^{\alpha, \pm}(\lambda, \tau)$ are non-increasing and left continuous functions with respect to $\lambda$; and they are equal to 0 when $\lambda>\lambda_{\infty}$ and equal to some constant when $\lambda \leq \lambda_{-\infty}$.

We take a cutoff function $\phi(\lambda) \in C_{0}^{\infty}(\mathbb{R})$ satisfying $0 \leq \phi \leq 1, \phi(\lambda)=1$ for $\left|\lambda-\lambda_{\infty}\right|<\delta_{0}$ and $\phi(\lambda)=0$ for $\left|\lambda-\lambda_{\infty}\right|>2 \delta_{0}$, and define $\overline{K_{N}^{0}}(\varphi)$ and $K_{N}^{-\infty}(\varphi)$ by

$$
\begin{aligned}
K_{N}^{0}(\varphi)= & \int_{\lambda_{-\infty}}^{\lambda_{\infty}} \int_{\mathbb{R}} e^{i \tau \lambda} i \tau \phi(\lambda)\left\{(i \tau)^{m} \tilde{\beta}_{0}(\lambda)+\sum_{j=1}^{N} \tilde{\beta}_{j}(\lambda, \tau)\right\} \hat{\varphi}(\tau) d \tau d \lambda, \\
K_{N}^{-\infty}(\varphi)= & \int_{\mathbb{R}} e^{i \tau \lambda_{-\infty}}\left\{(i \tau)^{m} \tilde{\beta}_{0}\left(\lambda_{-\infty}\right)+\sum_{j=1}^{N} \tilde{\beta}_{j}\left(\lambda_{-\infty}, \tau\right)\right\} \hat{\varphi}(\tau) d \tau \\
& +\int_{\lambda_{-\infty}}^{\lambda_{\infty}}(1-\phi(\lambda)) \int_{\mathbb{R}} e^{i \tau \lambda} i \tau\left\{(i \tau)^{m} \tilde{\beta}_{0}(\lambda)+\sum_{j=1}^{N} \tilde{\beta}_{j}(\lambda, \tau)\right\} \hat{\varphi}(\tau) d \tau d \lambda,
\end{aligned}
$$


where $\delta_{0}>0$ is a small number fixed later. Note that $K_{N}(\varphi)=-(2 \pi)^{-1}\left(K_{N}^{0}(\varphi)+\right.$ $\left.K_{N}^{-\infty}(\varphi)\right)$. It is easily seen that $K_{N}^{-\infty} \in C^{\infty}$ at $s=\lambda_{\infty}$. We put

$$
\gamma_{j}^{ \pm}(\lambda)=\frac{1}{2}\left\{\left.\left((i \tau)^{-m+j} \tilde{\beta}_{j}(\lambda, \tau)\right)\right|_{\tau=1} \pm\left.\left((i \tau)^{-m+j} \tilde{\beta}_{j}(\lambda, \tau)\right)\right|_{\tau=-1}\right\} .
$$

Noting that $\tilde{\beta}_{j}(\lambda, \tau)$ are homogeneous of order $m-j$ in $\tau$, we have $\gamma_{j}^{+}(\lambda)+\frac{\tau}{|\tau|} \gamma_{j}^{-}(\lambda)=$ $(i \tau)^{-m+j} \tilde{\beta}_{j}(\lambda, \tau)$. Thus, $K_{N}^{0}(\varphi)$ is of the form

$$
K_{N}^{0}(\varphi)=\int_{\lambda_{-\infty}}^{\lambda_{\infty}} \phi(\lambda)\left\{\tilde{\beta}_{0}(\lambda)+\sum_{j=1}^{N}\left(\gamma_{j}^{+}(\lambda)+i \gamma_{j}^{-}(\lambda) H\right)\left(\partial_{\lambda}^{-j}\right)\right\} \partial_{\lambda}^{m+1} \varphi(\lambda) d \lambda,
$$

where $H$ is the Hilbert transform: $\varphi \mapsto \int_{\mathbb{R}} e^{i \lambda \tau} \frac{-i \tau}{|\tau|} \hat{\varphi}(\tau) d \tau=\pi^{-1} \lim _{\epsilon \downarrow 0} \int_{|\mu| \geq \epsilon} \mu^{-1} \varphi(\lambda-$ $\mu) d \mu$. We set

$$
a_{j}^{ \pm}(\lambda)=\int_{\lambda_{\infty}}^{\lambda} \frac{(\lambda-\mu)^{j-1}}{(j-1) !} \gamma_{j}^{ \pm}(\mu) d \mu .
$$

Then $a_{j}^{ \pm}$belong to $C^{j-1}(\mathbb{R})$ and $\partial_{\lambda}^{j-1} a_{j}^{ \pm}$are absolutely continuous. Furthermore it follows that $a_{j}^{ \pm}(\lambda)=0$ for $\lambda>\lambda_{\infty}, \partial_{\lambda}^{l} a_{j}^{ \pm}\left(\lambda_{\infty}\right)=0(l=0,1, \ldots, j-1)$ and $\partial_{\lambda}^{j} a_{j}^{ \pm}(\lambda)=\gamma_{j}^{ \pm}(\lambda)$ almost everywhere. Therefore, applying integration by parts and using the skew self-adjoint property of the Hilbert transform, we obtain

$K_{N}^{0}(\varphi)=\int_{\mathbb{R}}\left\{\phi(\lambda) \tilde{\beta}_{0}(\lambda)+\sum_{j=1}^{N}(-1)^{j}\left(\phi(\lambda) a_{j}^{+}(\lambda)-i H\left(\phi a_{j}^{-}\right)(\lambda)\right\} \partial_{\lambda}^{m+1} \varphi(\lambda) d \lambda+\tilde{K}_{N}^{-\infty}(\varphi)\right.$,

where $\tilde{K}_{N}^{-\infty}(\varphi) \in \mathcal{D}^{\prime}(I)$ with $C^{\infty}$ at $\lambda=\lambda_{\infty}$. We take $\tilde{\phi}(\lambda) \in C_{0}^{\infty}(\mathbb{R})$ such that $0 \leq \tilde{\phi} \leq 1, \tilde{\phi}(\lambda)=1$ if $\left|\lambda-\lambda_{\infty}\right|<2 \delta_{0}$ and $\tilde{\phi}(\lambda)=0$ if $\left|\lambda-\lambda_{\infty}\right|>3 \delta_{0}$, and put

$$
\begin{aligned}
& \gamma(\lambda)=\gamma_{0}(\lambda)+i \sum_{j=1}^{N}(-1)^{j+1} \tilde{\phi}(\lambda) H\left(\phi a_{j}^{-}\right)(\lambda), \\
& \gamma_{0}(\lambda)=\phi(\lambda)\left\{\tilde{\beta}_{0}(\lambda)+\sum_{j=1}^{N}(-1)^{j} a_{j}^{+}(\lambda)\right\} .
\end{aligned}
$$

Since supp $[1-\tilde{\phi}] \cap \operatorname{supp}[\phi]=\emptyset$, it follows that $K_{N}^{0}-\tilde{K}_{N}^{0} \in C^{\infty}$ at $s=\lambda_{\infty}$, where $\tilde{K}_{N}^{0}(\varphi)=\int_{\mathbb{R}} \gamma(\lambda) \partial_{\lambda}^{m+1} \varphi(\lambda) d \lambda$. Thus, we obtain the following Lemma 7.3.

LEMma 7.3. For an integer $N>m+n+2$, the following (a) and (b) are equivalent to each other:
(a) $K \in H^{l}$ at $s=\lambda_{\infty}$,
(b) $\gamma \in H^{l+m+1}(\mathbb{R})$.

Since supp $[\eta] \cap E_{\lambda_{\infty}}(\subset V)$ is compact, from (7.1) we can choose an open set $W \subset$ $V$ and a constant $C_{0}>0$ such that $\operatorname{Re} \beta_{0}(x) \geq C_{0}$ on $W$ and supp $[\eta] \cap E_{\lambda_{\infty}} \subset W$. We also take a constant $\delta_{1}>0$ such that $\operatorname{supp}[\eta] \cap E_{\lambda} \subset W$ for $\lambda \geq \lambda_{\infty}-\delta_{1}$.

LEMMA 7.4. There exist an integer $k_{0}$ with $1 \leq k_{0} \leq n$, a constant $C_{1}>0$ and a constant $\delta_{2}>0$ with $\delta_{1}>\delta_{2}>0$ such that

$$
\int_{E_{\lambda}} \eta(x) d x \geq C_{1}\left(\lambda_{\infty}-\lambda\right)^{\frac{n}{2}-\frac{k_{0}}{4}} \quad\left(\lambda_{\infty}-\delta_{2} \leq \lambda \leq \lambda_{\infty}\right) .
$$


Proof. The matrix $A_{x_{0}}=\left(\partial_{x_{i}} \partial_{x_{j}} S\left(x_{0}\right)\right)_{i, j=1, \ldots, n}$ is non-positive since $S(x) \leq \lambda_{\infty}$ on $W$ and $\nabla_{x} S\left(x_{0}\right)=0$. We denote the positive eigenvalues of $-A_{x_{0}}$ by $\mu_{j}(j=$ $\left.1, \ldots, n-k_{0}(\leq n-1)\right)\left(\mu_{1} \geq \mu_{2} \geq \cdots \geq \mu_{n-k_{0}}\right)$. Note that $k_{0}=n$ if $A_{x_{0}}=0$. For $P \in O(n)$ we set $\tilde{S}(y)=S\left({ }^{t} P y+x_{0}\right)$. We can choose $P$ so that in a neighborhood of $y=0$

$$
\tilde{S}(y)-\lambda_{\infty}=-\sum_{j=1}^{n-k_{0}} \mu_{j} y_{j}^{2}+Q(y)+O\left(|y|^{4}\right),
$$

where $Q(y)=\sum_{|\alpha|=3} \frac{1}{\alpha !} \partial_{y}^{\alpha} S(0) y^{\alpha}$. We write $y={ }^{t}\left({ }^{t} y^{\prime},{ }^{t} y^{\prime \prime}\right), y^{\prime}={ }^{t}\left(y_{1}, \ldots, y_{n-k_{0}}\right)$ and $y^{\prime \prime}={ }^{t}\left(y_{n-k_{0}+1}, \ldots, y_{n}\right)$. Since $\tilde{S}\left(0, y^{\prime \prime}\right)-\lambda_{\infty} \leq 0$, we have $Q\left(0, y^{\prime \prime}\right)=0$, i.e., $\sum_{\left|\alpha^{\prime \prime}\right|=3} \frac{1}{\alpha^{\prime \prime \prime} !}\left(\partial_{y}^{\alpha^{\prime \prime}} S\right)(0) y^{\alpha^{\prime \prime}}=0\left(y^{\prime \prime} \in \mathbb{R}^{k_{0}}\right)$. Hence, there exists a constant $C>0$ such that

$$
Q(y) \geq-C\left\{\left|y^{\prime}\right|^{3}+\left|y^{\prime}\right|^{2}\left|y^{\prime \prime}\right|+\left|y^{\prime}\right|\left|y^{\prime \prime}\right|^{2}\right\} \geq-C\left\{3 \delta\left|y^{\prime}\right|^{2}+\frac{1}{2 \delta}\left|y^{\prime \prime}\right|^{4}\right\} \quad(|y| \leq \delta) .
$$

We can choose a small $\delta>0$ such that we have $W_{0}=\left\{x \in \mathbb{R}^{n} ; x=P^{-1} y+x_{0} \in\right.$ $\left.B_{\delta}\left(x_{0}\right)\right\} \subset \subset W\left(\right.$ where $\left.B_{\delta}\left(x_{0}\right)=\left\{y \in \mathbb{R}^{n} ;\left|y-x_{0}\right|<\delta\right\}\right)$ and

$$
\tilde{S}(y)-\lambda_{\infty} \geq-C^{\prime}\left\{\left|y^{\prime}\right|^{2}+\left|y^{\prime \prime}\right|^{4}\right\} \quad \text { in } \quad y \in B_{\delta}(0)
$$

for some constant $C^{\prime}>0$. We put $\delta_{2}=\min \left\{\delta_{1}, C^{\prime} \delta^{4} / 8,\left(2 C^{\prime}\right)^{-1}\right\}>0$, and put $W_{1}=\left\{y \in \mathbb{R}^{n} ;\left|y^{\prime}\right| \leq\left(2 C^{\prime}\right)^{-1 / 2}\left|\lambda_{\infty}-\lambda\right|^{1 / 2},\left|y^{\prime \prime}\right| \leq\left(2 C^{\prime}\right)^{-1 / 4}\left|\lambda_{\infty}-\lambda\right|^{1 / 4}\right\}$, where $\lambda_{\infty}-\delta_{2} \leq \lambda \leq \lambda_{\infty}$. From (7.7), it follows that $|y|<\delta$ and $\tilde{S}(y) \geq \lambda$ if $y \in W_{1}$. Taking $\delta>0$ to be sufficiently small again if necessary, we can obtain $\eta(x) \geq 2^{-1} \eta\left(x_{0}\right)>0$ for $x \in W_{0}$. Thus, if $\lambda_{\infty}-\delta_{2} \leq \lambda \leq \lambda_{\infty}$, we get

$$
\int_{E_{\lambda}} \eta(x) d x \geq \frac{1}{2} \eta\left(x_{0}\right) \int_{E_{\lambda} \cap W_{0}} d x \geq \frac{1}{2} \eta\left(x_{0}\right) \mathcal{V} o l\left(W_{1}\right) \geq C_{1}\left(\lambda_{\infty}-\lambda\right)^{\frac{n}{2}-\frac{k_{0}}{4}} .
$$

This completes the proof of Lemma 7.4.

Lemma 7.5. There exist constants $\delta_{3}>0$ and $C_{2}>0$ with $0<\delta_{3} \leq \delta_{2}$ such that

$$
\operatorname{Re} \gamma_{0}(\lambda) \geq C_{2} \phi(\lambda)\left(\lambda_{\infty}-\lambda\right)^{\frac{n}{2}-\frac{k_{0}}{4}} \quad\left(\lambda_{\infty}-\delta_{3} \leq \lambda \leq \lambda_{\infty}\right) .
$$

Proof. Since $\left|\gamma_{j}^{ \pm}(\lambda)\right| \leq \max _{x \in \bar{V}, \tau= \pm 1}\left|\beta_{j}(x, \tau)\right| \int_{E_{\lambda}} \eta(x) d x$, it follows from (7.5) that $\left|a_{j}^{ \pm}(\lambda)\right| \leq C_{N}\left|\lambda_{\infty}-\lambda\right|^{j} \int_{E_{\lambda}} \eta(x) d x(j=1,2, \ldots, N, \lambda \in \mathbb{R})$. Noing that $\operatorname{Re} \beta_{0}(x) \geq C_{0}\left(x \in W_{0}\right)$ and supp $[\eta] \cap E_{\lambda} \subset W_{0}\left(\lambda_{\infty}-\delta_{1} \leq \lambda \leq \lambda_{\infty}\right)$, we have $\operatorname{Re} \tilde{\beta}_{0}(\lambda) \geq C_{0} \int_{E_{\lambda}} \eta(x) d x\left(\lambda_{\infty}-\delta_{1} \leq \lambda \leq \lambda_{\infty}\right)$. Hence, we obtain Lemma 7.5 from Lemma 7.4 and the above estimates.

Proof of Theorem 7.1. We show Theorem 7.1 by the contradiction argument. Assume that $K \in H^{-m+\frac{n-1}{2}-\frac{k_{0}}{4}}$ at $\lambda=\lambda_{\infty}$ for $k_{0}$ as stated in Lemma 7.4. From Lemma 7.3, the function $\gamma(\lambda)$ in (7.6) belongs to $H^{\frac{n+1}{2}-\frac{k_{0}}{4}}(\mathbb{R})$. We put $s_{0}=\frac{n}{2}-\frac{k_{0}}{4}$. We then have $H \gamma_{0} \in H^{s_{0}+1 / 2}\left(\left(\lambda_{\infty}, \infty\right)\right)$ since $H \gamma_{0}(\lambda)=H \gamma(\lambda)+i \sum_{j=1}^{N}(-1)^{j} \phi(\lambda) a_{j}^{-}(\lambda)+$ $i \sum_{j=1}^{N}(-1)^{j}\left(H(\tilde{\phi}(\lambda)-1) H\left(\phi a_{j}^{-}\right)(\lambda)\right)$. Since $\gamma_{0}(\lambda)=0\left(\lambda>\lambda_{\infty}\right)$, we obtain $H \gamma_{0}(\lambda)=\pi^{-1} \int_{-\infty}^{\lambda_{\infty}}(\lambda-\mu)^{-1} \gamma_{0}(\mu) d \mu \in C^{\infty}\left(\left(\lambda_{\infty}, \infty\right)\right)$. We take $p \in \mathbb{N} \cup\{0\}$ and $\alpha_{0} \in[0,1)$ with $s_{0}+1 / 2=p+\alpha_{0}$ and define $f$ by $f(\lambda)=\int_{-\infty}^{\lambda_{\infty}}(\lambda-\mu)^{-1-p} \gamma_{0}(\mu) d \mu$. Then, we obtain $f \in C^{\infty}\left(\left(\lambda_{\infty}, \infty\right)\right) \cap H^{\alpha_{0}}\left(\left(\lambda_{\infty}, \infty\right)\right)$. 
We fix $\delta_{0}>0$ with $\delta_{0}<\delta_{3} / 2$ and put $g(s)=f\left(\lambda_{\infty}+s\right)$ and $k(\tau)=\gamma_{0}\left(\lambda_{\infty}-\tau\right)$. Then, we get $g \in C^{\infty}\left(\mathbb{R}_{+}\right) \cap H^{\alpha_{0}}\left(\mathbb{R}_{+}\right)\left(\mathbb{R}_{+}=(0, \infty)\right), g(s)=\int_{0}^{\infty}(s+\tau)^{-1-p} k(\tau) d \tau$ $(s>0)$, Re $k \geq 0$ in $\mathbb{R}_{+}, \operatorname{Re} k(\tau) \geq C_{2} \tau^{p+\alpha_{0}-1 / 2}\left(0 \leq \tau \leq \delta_{0}\right)$ and $k(\tau)=0(0>\tau$ or $\tau \geq 2 \delta_{0}$ ). Using Lemma 7.5, we now show the contradiction in each of the cases for $\alpha_{0}=0$ and $0<\alpha_{0}<1$.

The case of $\alpha_{0}=0$ : Since $s+\tau \leq 2 \tau$ if $0 \leq s \leq \tau$, for $s$ at $0<s \leq \delta_{0}$ we have

$$
\operatorname{Re} g(s) \geq \int_{s}^{\delta_{0}} \frac{C_{2} \tau^{p-1 / 2}}{(2 \tau)^{p+1}} d \tau \geq C_{2} 2^{-p}\left(s^{-1 / 2}-\delta_{0}^{-1 / 2}\right) .
$$

This means $g \notin L^{2}\left(\mathbb{R}_{+}\right)$, which is contradictory.

The case of $0<\alpha_{0}<1$ : Since we have $s+t+\tau \leq 3 \tau$ and $s+\tau \leq 2 \tau$ if $0 \leq t \leq s \leq$ $\tau \leq \delta_{0}$, noting that $(s+t+\tau)^{p+1}-(s+\tau)^{p+1} \geq(p+1) t(s+\tau)^{p}$, we have

$$
\begin{aligned}
-\operatorname{Re}(g(t+s)-g(s)) & \geq \int_{0}^{\delta_{0}} \frac{C_{2}(p+1) t(s+\tau)^{p} \tau^{p+\alpha_{0}-1 / 2}}{(s+t+\tau)^{p+1}(s+\tau)^{p+1}} d \tau \\
& \geq \frac{C_{2}(p+1)}{3^{p+1} 2} t \int_{s}^{\delta_{0}} \tau^{\alpha_{0}-2-1 / 2} d \tau
\end{aligned}
$$

if $0<t \leq s \leq \delta_{0}$. Hence, there exists a constant $C_{3}>0$ such that

$$
-\operatorname{Re}(g(t+s)-g(s)) \geq C_{3} t\left(s^{\alpha_{0}-3 / 2}-\delta_{0}^{\alpha_{0}-3 / 2}\right) \quad\left(0<t \leq s \leq \delta_{0}\right),
$$

which implies that if $0<t<\delta_{0}$,

$\|g(t+\cdot)-g(\cdot)\|_{L^{2}\left(\mathbb{R}_{+}\right)}^{2} \geq C_{3}^{2} t^{2} \int_{t}^{\delta_{0}}\left|s^{\alpha_{0}-3 / 2}-\delta_{0}^{\alpha_{0}-3 / 2}\right|^{2} d s \geq \frac{C_{3}^{2}}{4\left(1-\alpha_{0}\right)}\left(t^{2 \alpha_{0}}-9 \delta_{0}^{2 \alpha_{0}-2} t^{2}\right)$.

This estimate implies that $\int_{0}^{\infty} t^{-\left(2 \alpha_{0}+1\right)}\|g(t+\cdot)-g(\cdot)\|_{L^{2}\left(\mathbb{R}_{+}\right)}^{2} d t<\infty$ does not hold. Hence, we get $g \notin H^{\alpha_{0}}\left(\mathbb{R}_{+}\right)$, which is contradictory. Thus we obtain Theorem 7.1.

Proof of Theorem 7.2. We take $\delta_{x_{0}} \geq 0$ to be sufficiently small again if necessary. Then, noting that $S(x)-\lambda_{\infty} \leq 0(x \in \bar{V})$, we obtain

$$
\begin{aligned}
& S(x)-\lambda_{\infty}=\sum_{\left|\alpha^{\prime}\right|=2 l_{0}+2} \frac{1}{\alpha^{\prime} !} \partial_{x^{\prime}}^{\alpha^{\prime}} S\left(x_{0}^{\prime}, x^{\prime \prime}\right)\left(x^{\prime}-x_{0}^{\prime}\right)^{\alpha}+G(x), \\
& |G(x)| \leq C\left|x^{\prime}\right|^{2 l_{0}+3} \quad\left(x \in \tilde{B}\left(x_{0}, \delta_{x_{0}}\right) \subset W\right),
\end{aligned}
$$

where $\tilde{B}\left(x_{0}, \delta_{x_{0}}\right)=\left\{x \in \mathbb{R}^{n} ;\left|x^{\prime}-x_{0}^{\prime}\right|<\delta_{x_{0}},\left|x^{\prime \prime}-x_{0}^{\prime \prime}\right|<\delta_{x_{0}}\right\}$ and $W$ is an open set satisfying $\operatorname{supp}[\eta] \cap E_{\lambda} \subset W$ for $\lambda \geq \lambda_{\infty}-\delta_{1}$. From this expansion, it follows that

$$
S(x)-\lambda_{\infty} \geq-C^{\prime}\left|x^{\prime}-x_{0}^{\prime}\right|^{2\left(l_{0}+1\right)} \quad\left(x \in \tilde{B}\left(x_{0}, \delta_{x_{0}}\right)\right) .
$$

We put $\delta_{2}^{\prime}=\min \left\{\delta_{1}, C^{\prime}\left(\delta_{x_{0}} / 2\right)^{2\left(l_{0}+1\right)}\right\}>0$. Since $\left|x^{\prime}-x_{0}^{\prime}\right|<\delta_{x_{0}}$ if $\left|x^{\prime}-x_{0}^{\prime}\right| \leq$ $\left(C^{\prime}-1\left|\lambda_{\infty}-\lambda\right|\right)^{\frac{1}{2\left(l_{0}+1\right)}}$ and $\lambda_{\infty}-\delta_{2}^{\prime} \leq \lambda \leq \lambda_{\infty}$, we have $S(x) \geq \lambda$. Hence, in the same way as in the proof of Lemma 7.4, we have

$$
\int_{E_{\lambda}} \eta(x) d x \geq C_{1}^{\prime}\left(\lambda_{\infty}-\lambda\right)^{\frac{n-k_{1}}{2\left(l_{0}+1\right)}} \quad\left(\lambda_{\infty}-\delta_{2}^{\prime} \leq \lambda \leq \lambda_{\infty}\right) .
$$


Therefore, using the same argument as in Lemma 7.5, we obtain

$$
\operatorname{Re} \gamma_{0}(\lambda) \geq C_{2}^{\prime} \phi(\lambda)\left(\lambda_{\infty}-\lambda\right)^{\frac{n-k_{1}}{2\left(l_{0}+1\right)}} \quad\left(\lambda_{\infty}-\delta_{3}^{\prime} \leq \lambda \leq \lambda_{\infty}\right)
$$

for some constants $C_{2}^{\prime}>0$ and $\delta_{3}^{\prime}>0$. Thus, we can obtain (i) in Theorem 7.2 by the same arguments as in Theorem 7.1.

We now show (ii) in Theorem 7.2. Since $a_{j}^{ \pm} \in C^{j-1}(\mathbb{R})$ and $\partial_{\lambda}^{j} a_{j}^{ \pm}(\lambda)=\gamma_{j}^{ \pm}(\lambda)$ almost everywhere, it follows that $\phi a_{j}^{ \pm} \in H^{1}(\mathbb{R})$. Hence it suffices to show that $\phi \tilde{\beta}_{0} \in H^{\frac{1}{2\left(l_{0}+1\right)}+\frac{1}{2}-\epsilon_{0}}(\mathbb{R})$ for any $\epsilon_{0}>0$. In the same way as for (7.4), we obtain

$$
-i \tau \int_{\mathbb{R}} e^{i \tau \lambda} \phi(\lambda) \tilde{\beta}_{0}(\lambda) d \lambda=\int_{U} e^{i \tau S(x)} \eta(x) \phi(S(x)) \beta_{0}(x) d x .
$$

Using the same arguments as for (7.8), we can show from the assumption (7.3) that for $x^{(1)} \in E_{\lambda_{\infty}}$ there exist constants $\delta_{x^{(1)}}>0$ and $C_{0}\left(x^{(1)}\right)>0$ such that

$$
\begin{aligned}
& S(x)-\lambda_{\infty}=\frac{1}{\left(2 l_{0}+2\right) !} \partial_{x_{1}}^{2\left(l_{0}+1\right)} S\left(x_{1}^{(1)}, x^{\prime \prime}\right)\left(x_{1}-x_{1}^{(1)}\right)^{2\left(l_{0}+1\right)}+\tilde{G}\left(x_{1}, x^{\prime \prime}\right), \\
& \left|\tilde{G}\left(x_{1}, x^{\prime \prime}\right)\right| \leq C_{0}\left(x^{(1)}\right)\left|x_{1}-x_{1}^{(1)}\right|^{2 l_{0}+3} \quad\left(x \in \tilde{B}\left(x^{(1)}, \delta_{x^{(1)}}\right)\right) .
\end{aligned}
$$

Note that $\partial_{x_{1}}^{2\left(l_{0}+1\right)} S\left(x_{1}^{(1)}, x^{\prime \prime}\right)<0\left(\left|x^{\prime \prime}-\left(x^{(1)}\right)^{\prime \prime}\right|<\delta_{x^{(1)}}\right)$ follows from $S(x) \leq \lambda_{\infty}$ $(x \in \bar{V})$. Then, we obatin

$$
\begin{aligned}
& S(x)=\lambda_{\infty}-A\left(x, x^{(1)}\right)\left(x_{1}-x_{1}^{(1)}\right)^{2\left(l_{0}+1\right)} \quad\left(x \in \tilde{B}\left(x^{(1)}, \delta_{x^{(1)}}\right)\right), \\
& A\left(\cdot, x^{(1)}\right) \in C^{\infty}\left(\tilde{B}\left(x^{(1)}, \delta_{x^{(1)}}\right)\right), \quad A\left(x, x^{(1)}\right)>0 \quad\left(x \in \tilde{B}\left(x^{(1)}, \delta_{x^{(1)}}\right)\right) .
\end{aligned}
$$

From compactness of supp $[\eta] \cap E_{\lambda_{\infty}}$, we can take $x^{(1)}, x^{(2)}, \ldots, x^{(\tilde{N})} \in E_{\lambda_{\infty}}$ such that $\operatorname{supp}[\eta] \cap E_{\lambda_{\infty}} \subset \cup_{j=1}^{\tilde{N}} \tilde{B}\left(x^{(j)}, \delta_{x^{(j)}} / 3\right)$. We put $\tilde{W}=\cup_{j=1}^{\tilde{N}} \tilde{B}\left(x^{(j)}, 2 \delta_{x^{(j)}} / 3\right)$. Since $S(x)$ is continuous on $\bar{V}$, there exists a constant $\delta_{4}>0$ such that $\operatorname{supp}[\eta] \cap E_{\lambda} \subset \tilde{W}$ for $\lambda$ with $\lambda_{\infty}-\delta_{4} \leq \lambda \leq \lambda_{\infty}$. We now choose the cutoff function $\phi$ introduced in the proof of Lemma 7.3, and change $\delta_{0}>0$ there to $\delta_{0}=\delta_{4} / 2>0$. Furthermore, we take a partition $\left\{\psi_{j}\right\}_{j=1,2, \ldots, \tilde{N}}$ of unity on $\tilde{W}$ such that $\psi_{j} \in C^{\infty}\left(\tilde{B}\left(x^{(j)}, \delta_{x^{(j)}}\right)\right)$, $0 \leq \psi_{j} \leq 1(j=1,2, \ldots, \tilde{N})$. Changing the variable $\xi=A\left(x, x^{(j)}\right)^{\frac{1}{2\left(l_{0}+1\right)}}\left(x_{1}-x_{1}^{(j)}\right)$ for each $j$, and noting that $\phi(S(x))=\sum_{j=1}^{\tilde{N}} \phi(S(x)) \psi_{j}(x)$, we can show that for each $j$ there exists $b_{j} \in C_{0}^{\infty}\left(\mathbb{R}^{n}\right)$ such that

$$
-i \tau\left(\phi \tilde{\beta}_{0}\right)^{\wedge}(\tau)=\sum_{j=1}^{\tilde{N}} e^{i \lambda_{\infty} \tau} \int_{\mathbb{R}^{n-1}} \int_{\mathbb{R}} e^{-i \tau \xi^{2\left(l_{0}+1\right)}} b_{j}\left(\xi, x^{\prime \prime}\right) d \xi d x^{\prime \prime} .
$$

Hence, we obtain $\left|\left(\phi \tilde{\beta}_{0}\right)^{\wedge}(\tau)\right| \leq C(1+|\tau|)^{-1-\frac{1}{2\left(l_{0}+1\right)}}(\tau \in \mathbb{R})$. This means $\phi \tilde{\beta}_{0} \in$ $H^{\frac{1}{2\left(l_{0}+1\right)}+\frac{1}{2}-\epsilon_{0}}(\mathbb{R})$, which completes proof of Theorem 7.2 .

\section{REFERENCES}

[1] J. D. Achenbach, Wave Propagation in Elastic Solids, North-Holland, New York, 1973.

[2] L. Hörmander, The Analysis of Linear Partial Differential Operators III, Springer, New York, 1985. 
[3] W. ICHINose, The Cauchy problem for Schrödinger type equations with variable coefficients, Osaka J. Math., 24 (1987), pp. 853-886.

[4] W. ICHINose, On $L^{2}$-well posedness of the Cauchy problem for Schrödinger type equations on the riemannian manifold and the Maslov theory, Duke Math. J., 56 (1988), pp. 549-588.

[5] M. KaWASHITA, On the local-energy decay property for the elastic wave equation with the Neumann boundary condition, Duke J. Math., 67 (1992), pp. 333-351.

[6] M. Kawashita, On a region free from the poles of the resolvent and decay rate of the local energy for the elastic wave equation, Indiana Univ. Math. J., 43 (1994), pp. 1013-1043.

[7] M. Kawashita, W. Kawashita AND H. Soga, Relation between scattering theories of the Wilcox and Lax-Phillips types and a concrete construction of the translation representation, Comm. P. D. E., 28 (2003), pp. 1437-1470.

[8] M. Kawashita, W. Kawashita And H. Soga, Scattering theory for the elastic wave equation in perturbed half-spaces, Trans. Amer. Math. Soc., 358 (2006), pp. 5319-5350.

[9] H. Kumano-Go, Pseudo-differential operators, MIT Press, Cambridge, 1981.

[10] P. D. Lax AND R. S. Phillips, Scattering theory, Academic Press, New York, 1967.

[11] A. MAJDA, A Representation formula for the scattering operator and the inverse problem for arbitrary bodies, Comm. Pure Appl. Math., 30 (1977), pp. 165-194.

[12] V. P. Maslov and M. V. Fedoriuk, Semi-classical Approximation in Quantum Mechnics, D. Reidel, Dordrecht, 1981.

[13] M. Matsumura, Asymptotic behavior at infinity for Green's functions of first order systems with characteristics of nonuniform multiplicity, Publ. Res. Inst. Math., 12 (1976), pp. 317377.

[14] H. SogA, Singular support of the scattering kernel for the acoustic equation in inhomogeneous media, Comm. PDE, 9 (1984), pp. 467-502.

[15] H. SogA, Condition against rapid decrease of oscillatory integrals and their applications to inverse scattering problems, Osaka Math. J., 23 (1986), pp. 441-456.

[16] H. SogA, Representation of the scattering kernel for the elastic wave equation and singularities of the back-scattering, Osaka J. Math., 29 (1992), pp. 809-836.

[17] P. Stefanov and G. Vodev, Distribution of the resonances for the Neumann problem in linear elasticity in the exterior of a strictly convex body, Duke Math. J., 78 (1995), pp. 677-714.

[18] P. Stefanov and G. Vodev, Neumann resonances in linear elasticity for an arbitrary body, Comm. Math. Phys., 176 (1996), pp. 645-659.

[19] M. TAYLOR, Rayleigh waves in linear elasticity as a propagation of singularities phenomenon, In Proc. Conf. on PDE and Geometry Marcel Dekker New York 1979, pp. 273-291.

[20] M. TAYlor, Pseudo-differential operators, Princeton Univ. Press, Princeton, N.J., 1981. 
M. KAWASHITA AND H. SOGA 